\title{
Abstracts: EPSM-ABEC 2009
}

\section{Engineering and Physical Sciences in Medicine \& the Australian Biomedical Engineering College Conference, 8-12 November 2009, Hotel Realm, Canberra, ACT}

\section{Session 1-Opening plenary session}

Back to the future in health care technology

\author{
James P Keller, $\mathbf{J r}^{1}$ \\ ${ }^{1}$ Health Technology Evaluation and Safety, ECRI Institute
}

Let's take a tour to the year 2035 to explore our exciting new world of health care technology. The trip begins with an unfortunate accident suffered by Marty McFly (aka Michael J Fox) as he crash-landed his Delorean time machine onto the round-about in the centre of Coober Pedy, South Australia. Marty suffered serious injuries from an over 500 mile per hour impact caused by the blast from his transition through the 'space-time' continuum. Marty's accident was immediately responded to by a mobile emergency response hospital (MERH) that began treatment on his many injuries. This presentation will follow Marty's path through the health care system of 2035 on his long road to recovery. Learn about the new technology used to save his life and that interestingly helped diagnose and treat an early onset condition of Parkinson's disease.

Health care technology has gone through a sea change from present day to the year 2035. Much of the change was precipitated by the American Recovery and Reinvestment Act of 2009 and the Edward Kennedy US Healthcare Reform Act of 2010. Despite Marty McFly's time travel 25 years into the future, the sophisticated health care information systems of 2035 were able to locate his health records from 2009. This information was first requested from his MERH caregivers to identify any underlying conditions that might affect his initial care.

Many health care workers supported Marty's care during his travel to the year 2035. This presentation will touch on the roles played by doctors, nurses, and other caregivers as he was literally pieced back together from his severe injuries. But it will focus more on new roles for the dedicated set of professionals that developed and supported the technologies used to monitor his progress throughout his care and that now hold him back together in 2009 .

\section{Session 2A-Nuclear medicine and radiotherapy}

Back to the future in nuclear medicine

Dale Bailey ${ }^{1}$

${ }^{1}$ Department of Nuclear Medicine, Royal North Shore Hospital, and University of Sydney
In the first film in the Back to the Future trilogy, the unfortunate time traveller is transported 30 years back in time with the potential impact of changing the course of his personal history. If I were to be transported back 30 years in time, how would I seek to alter the course of history in the developments in physics in Nuclear Medicine? I would start by considering the impact of changes in three areas: instrumentation developments, research, and education and training. Where have we succeeded and where have we failed? Finally, how does this frame where we should be aiming in the future?

In the area of instrumentation, the traditional lead collimator used with the gamma camera has proven to be the albatross around the neck of single photon imaging with radionuclides. However, there are a number of new developments which are aiming to enhance sensitivity as well as resolution. Multi-pinhole technology has been around for over 30 years but has been mostly neglected. Now, a dedicated cardiac imaging camera, using solid state detectors and multi-pinhole collimators is available. Sensitivity gains of eight to tenfold have been reported on this device. This path was available in the past, but was never pursued. Eventually, I anticipate that the lead collimator will be replaced by an electronic one, à la the Compton telescope used on satellites which uses a photon scattering solid state front detector and a more conventional rear detector, minus the collimator. In PET, instrumentation developments have been driven by oncological applications using $\left[{ }^{18} \mathrm{~F}\right]-\mathrm{FDG}$, a glucose analog, for around a decade now. Much of the emphasis has focused on improving throughput. While this has had an enormous impact on managing cancer patients, it means that one of the strengths of PET, namely studying physiologically pathways such as neurotransmitters and endocrine/paracrine interactions have been largely neglected. On the positive side, this means that there is still enormous scope for active pursuit by dedicated physicists, physiologists and physicians and provides a rich field to explore in understanding the more complicated 'systems' disorders and interactions between seemingly different physiological processes. One of our major problems in this area is communication-can we talk the same language as the researchers asking the big questions in these complicated fields? Why hasn't the rapid development of the field of biomarkers made more of an impact?

In research, it has been argued elsewhere that imaging with radiotracers has 'punched below its weight' for many years and should be a more cutting edge tool based on huge advances in molecular technologies such as increased genomic information and 
synthetic chemistry. However, I see the future as being one where information from a range of diverse sources, including both imaging and non-imaging data, is integrated. PET/CT and SPECT/ CT are common clinical tools now, and MRI combined with PET and SPECT is looming on our medical imaging event horizon. Why was the combination of radionuclide/functional and structural/ anatomical imaging not envisaged earlier? Combining X-rays with nuclear medicine scans has been reported since the mid-1960s, but it took an unusual set of circumstances to produce the combined $\mathrm{PET} / \mathrm{CT}$ which has revolutionised imaging in the past 10 years. We should ask what potential synergies are now staring us in the face, working independently of each other. Who is "pushing the outer envelope' today? Imaging photons emitted from heavy ion therapy? Guiding MV radiation therapy based on feedback about cellular kinetics?

Finally, in education and training I believe we have made much progress. 30 years ago in Sydney it was hardly possible to undertake a $\mathrm{PhD}$ in medical physics due to lack of adequate academic supervision. That has changed for the better. But what is the purpose of our education and training today? Do we train our students and trainees to think, or merely not to make potentially life-threatening mistakes? How will medical physics respond to the government's 'education revolution'-how will we revolt in our training programs? How will our roles and training change to accommodate the information and instrumentation we need to dream of developing?

Finally, I leave the reader with a question: if I gave you $\$ 100 \mathrm{~m}$ research money for blue sky activities, where would you invest it? The distance of your vision for the future may reflect on the approach you take today to the areas discussed above.

\section{Respiratory motion management in radiotherapy: past, present and future}

\section{Sastry Vedam ${ }^{1}$ \\ ${ }^{1}$ University of Texas, USA}

Respiratory motion introduces geometric and dosimetric uncertainties during all stages (simulation, treatment planning and treatment delivery) of radiotherapy of tumour volumes, especially in the thorax and abdomen. Such uncertainties contain both systematic and random components and occur both within (intra) and between (inter) fractions of radiation delivery. Historically, such uncertainties have been accounted for by the addition of population based safety margins. This "one-size-fits-all" approach to addressing geometric uncertainties usually resulted in greater volumes of surrounding normal tissue receiving high dose radiation, thereby adversely affecting treatment outcomes.

In the past decade, advances in respiratory motion management technology, such as respiratory-correlated/4D CT imaging, have made patient specific assessment of intra-fraction tumour motion possible. Based on such assessment, several management strategies such as motion accommodation, breath hold, respiratory gating and real-time tumour tracking based delivery methods have been developed. Simultaneously, in the last decade, several advances have been made in integrating image guidance technology into clinical radiotherapy practice. Prominent examples include on board imaging, cone beam $\mathrm{CT}$ and digital tomosynthesis. The combination of image guidance and respiratory motion management therefore offers the greatest degree of reduction in geometric and dosimetric uncertainties during radiotherapy.

This presentation traces the history of the development of respiratory motion management in the field of radiotherapy, its current applications, benefits and risks and also attempts to highlight potential future applications and advancements.

\section{Session 2B-Health care technologies of the future}

\author{
Health Care Technology 2020-Health technology for dummies: \\ a guide to managing the future
}

\section{GJ FitzGerald ${ }^{1}$}

${ }^{1}$ Queensland University of Technology

Health technology and its disciple e-health is a broad church with the usual array of sects, temples of wisdom, a sprinkling of terrorists and much more. But as often happens, the core principles or tenets are sometimes lost in the politics of the devout; confused by jargon and held ransom by ritual, secret codes and handshakes. This helps to ensure power and control for the custodians of the mystery; for those who do, or think they understand, but who often lack the capacity to ensure the translation of discovery into practice.

There is a vast array of activity. A simple Google search of e-health provided a mere 2,110,000 entries while health technology produced $874 \mathrm{~m}$ entries. We have e-health research centres Professors of e-health and health technology, text books, journals and conferences yet the average health manager, who ultimately holds the accountability, is often left stranded a wake of confusion and disorientation.

If we are to progress the health technology agenda, then health managers need to seize control from the technophiles or at least enter into a meaningful relationship. We need to understand the complexity in order to manage it. We need to identify the clinical and business needs of health and explore the capacity of health technology to meet those needs not vice versa. It is the 'health' not the 'technology' part that is important.

This paper represents the attempt by a technology ignoramus to identify a conceptual framework for health technology, to propose a disciplining of the language and 'strategies' for future evaluation and development. I would like to share a vision of the future; a vision fortunately limited by a lack of imagination, but a vision which therefore requires little more of technology than that which many industries have imbedded for more than 10 years. I would also like to share a view of the pathway to that vision and to propose a higher order approach.

Ask not what your health technology can do for you.

Ask what you need your health technology to achieve.

\section{Health technology-the future}

\section{Marc Lakos ${ }^{1}$ \\ ${ }^{1} 1$ st Health Support Battalion}

New health care technologies impact on clinical care, the patient experience, workforce requirements, facility design and health care costs. While consumers become excited at the promise of longer healthier lives, regulatory bodies are often unable to moderate demand and the competition for health resources escalate. Strategies for the rational implementation of new health care technologies are required. This can only be achieved when researchers, engineers, consumers and health administrators come together and make evidence-based decisions that deliver quality health care within a costeffective health care system. 


\section{Lunch session}

Crisis in health care financing in North America: a medical physicist's perspective

\section{Ervin B Podgorsak ${ }^{1}$ \\ ${ }^{1}$ McGill University, Canada}

The United States and Canada are very similar in social and economic development, but differ significantly in their approach to health care financing. Canada is committed to universal, publicly funded health insurance and spends $10 \%$ of its GNP (\$3900 per capita) annually on health care; the US relies largely on employment-related private health insurance, limits government involvement in health care only to coverage for the elderly, disabled, and poor and, despite spending $16 \%$ of its GNP (\$7900 per capita) annually on health care, leaves about $15 \%$ of its citizens without health insurance. Both countries are experiencing problems with escalating health care costs and accessibility, albeit for different reasons.

The main issue in the US is how to use government intervention to make the current health care system universal and more costeffective, while in Canada the health care system suffers serious problems with access to physicians and high technology equipment. However, in comparison with the American system, the Canadian system nonetheless produces better results and, in addition, is socially and morally just, equitable, and significantly cheaper.

Australia's health care system is publicly run and similar to that of Canada. However, at $8.7 \%$ of GNP ( $\$ 3150$ per capita), it is less costly than the Canadian system and despite its lower cost, it outperforms both the Canadian and the American system on standard health care indicators, such as life expectancy at birth, infant mortality, and maternal mortality at birth.

The example of the US, Canada, and Australia shows that, while market forces produce the most efficient economic system, health care should not be treated like an ordinary market commodity. In comparison with private health care systems, the publicly administered systems produce better results and at a lower cost; however, there are also pronounced differences in performance and cost from one public system to another.

\section{Session 3A-Radiotherapy}

\section{Consideration of temporary intra-fraction prostate movement during external beam radiotherapy}

Tomas Kron ${ }^{1}$, Jessica Thomas $^{1}$, Chris Fox ${ }^{1}$, Alan Herschtal ${ }^{1}$, Rebecca Owen ${ }^{1}$, Richard Oates ${ }^{1}$, Paul Roxby ${ }^{1}$, Farshad Foroudi ${ }^{1}$

${ }^{1}$ Peter MacCallum Cancer Centre

Background and aim The introduction of daily image guidance has reduced interfraction variation in prostate location significantly. Therefore, intrafraction motion has become more important in determining the size of margins required for external beam radiotherapy. Simplified, intrafraction motion consists of two components, a slow continuous movement (e.g. due to bladder filling) and temporary excursions (e.g. passing of rectal gas). We aimed to model if more frequent imaging such as EPI for each treatment field can be used to obtain reliable information on prostate excursions.

Methods A spreadsheet was developed with parameters that describe treatment delivery (beam-on time, number of fields and fractions), the excursion (duration and frequency) and the maximal allowable dose deviation assuming each excursion will result in underdose of the target. Poisson statistics was used to describe the probability of an excursion occurring during beam delivery.

Different beam-on times and fraction numbers were used to mimic IMRT and hypofractionated treatments.

Results Using typical assumptions for beam delivery in conformal radiotherapy not more than one excursion per 3 min can be allowed before the dose to the patient is reduced by more than $5 \%$. The longer beam-on times for IMRT make the treatment less sensitive to brief excursions while hypofractionation increases the sensitivity slightly as the overall dose is reduced. Using an EPI in all fields of the first week to assess motion of fiducial markers gives a $75 \%$ probability of detecting this frequency of excursions.

Conclusion Utilising EPI during treatment delivery may be a viable option to assess intra-fraction excursions of the prostate during radiotherapy.

\section{In-vivo rectal wall dosimetry for radiotherapy}

\author{
LE Cartwright $^{1,2}$, N Suchowerska ${ }^{1,2}$, Y Yin $^{1}$, J Lambert $^{1}$, \\ M Jackson $^{2}$, DR McKenzie ${ }^{1}$ \\ ${ }^{1}$ School of Physics, The University of Sydney; ${ }^{2}$ Department \\ of Radiation Oncology, Royal Prince Alfred Hospital
}

Radiation therapy clinical practice has a long history of success in treating gynaecological and prostate cancers. The rectum is close to both of these treatment sites, making rectal damage a key limiting factor in pelvic radiation treatment. To spare the healthy rectum, clinicians constrain the prescribed radiation dose and treatment technique in pelvic radiotherapy.

Despite compelling evidence showing the benefits of increased radiation dose for prostate patients (Zelefsky 2000), current treatment practice is conservative because of the need to preserve the rectum. The uncertainty in the dose received by the rectum throughout the whole treatment is a barrier to dose escalation and therefore to improvement of patient outcomes. A method to determine the true rectal wall dose is a prerequisite to optimal radiation therapy treatment. The aim of this study is to develop a complete dosimetry system customised for measurement of dose to the rectal wall. This innovation will provide a real-time dose map for the rectum, enabling definitive assessment of the treatment and intervention when needed.

An array of 16 scintillation dosimeters, known as fibre optic dosimeters $\left(\mathrm{FOD}^{\mathrm{TM}}\right)$ in an insertable applicator has been designed and built. The dosimeters were calibrated simultaneously in a custom built circular jig before use. Each dosimeter is optically interfaced to a set of pixels on a CCD camera located outside the treatment bunker. Customised software accumulates dose into 16 channels for real time display while the treatment is delivered. The performance of the array was demonstrated by simulating brachytherapy treatments in a water phantom. The measured doses were compared to those predicted by the treatment plan and found to be in agreement to within the uncertainty of measurement. The array was also used to track the progression of the source as it moved along the catheter. The measured position was found to agree with the position reported by the HDR system to within the measurement uncertainty.

This work lays the foundation for rectal wall in vivo measured dose mapping in a form accessible to the clinician during and after treatment. The dose map calculated by the treatment planning system would be presented along with the FOD ${ }^{\mathrm{TM}}$ measurements superimposed as demonstrated in our phantom implementation. The in vivo measurements will inform the clinician at the completion of treatment as to whether the prescribed treatment has actually been delivered. The use of the rectal FOD ${ }^{\mathrm{TM}}$ array will provide an early warning of a potential radiation accident, provide real time monitoring of treatment delivery and provide a record of the actual radiation dose delivered, 
which can be used in a database to correlate rectal complications with the actual pattern of dose deposition.

Acknowledgments The authors acknowledge funding from the NHMRC in support of this research.

\section{A Silicon Strip Detector Dose Magnifying Glass for IMRT Dosimetry}

J.H.D. Wong ${ }^{1,5}$, M. Carolan ${ }^{1,2}$, M.L.F. Lerch ${ }^{1}$, M. Petasecca ${ }^{1}$, S. Khanna ${ }^{3}$, V.L. Perevertaylo ${ }^{4}$, P. Metcalfe ${ }^{1}$, \& A.B. Rosenfeld ${ }^{1}$

${ }^{1}$ Centre for Medical Radiation Physics, University of Wollongong, NSW 2522, Australia, ${ }^{2}$ Illawarra Cancer Care Centre, Wollongong Hospital, NSW 2500, Australia, ${ }^{3}$ St. George Cancer Care Centre, Kogarah, Sydney, NSW 2217, Australia, ${ }^{4}$ SPA BIT, Ukraine,

${ }^{5}$ Faculty of Medicine, University of Malaya, Kuala Lumpur 50603, Malaysia

Introduction Intensity modulated radiation therapy (IMRT) allows the delivery of escalated radiation dose to tumor while sparing adjacent critical organs. In doing so, IMRT plans tend to incorporate steep dose gradients. The CMRP DMG is a high spatial resolution $(200 \mathrm{~m})$ silicon strip detector suitable for measuring the high dose gradients in an IMRT delivery.

Methods A full characterization of the detector was performed, including dose rate effect, PDD, stem effect, dose linearity, doseper-pulse, uniformity, energy response, angular response, and penumbra measurements. We also present the application of the CMRP DMG in the dosimetric verification of a clinical IMRT plan.

Results The DMG showed dose rate dependence and a correction function was derived to correct for this effect. The DMG PDD matches well with the Farmer ion chamber with $0.8 \%$ difference at $20 \mathrm{~cm}$ depth. Stem effect was negligible and dose linearity was excellent for the dose range of 3-300 cGy. For a change of 390-fold of dose-perpulse, detector response changed by $23 \%$. Uniformity was $<0.2 \%$ after uniformity correction. The detector showed an over-response relative to tissue dose at lower photon energies. Penumbra measured at $\mathrm{d}_{\max }$ for $6 \mathrm{MV}$ were $2.77 \mathrm{~mm}$ (secondary collimators) and $3.52 \mathrm{~mm}$ (MLC). The average difference of point doses measured in an IMRT plan measured with DMG versus EBT film and doses predicted by treatment planning system was better than $1.1 \%$. We demonstrated the high temporal resolution capability of the readout system.

Conclusions The CMRP silicon strip detector dose magnifying glass with its high spatial and temporal resolution is a valuable tool for QA in IMRT and VMAT dose delivery.

\section{BrainLab ExacTrac patient alignment data review}

\section{Trevor Ackerly ${ }^{1}$, Craig Lancaster ${ }^{1}$, Kathleen Roxby ${ }^{1}$ \\ ${ }^{1}$ William Buckland Radiotherapy Centre, The Alfred}

Introduction The William Buckland Radiotherapy Centre installed the BrainLab ExacTrac frameless stereotactic system in March 2008, and has since treated over 230 patients. The accuracy of the system depends on operator reviewed automatic planar image fusion between in-room kilovoltage images and CT-derived DRRs.

Methods The 6D corrections from all patients were analysed. Standard clinical procedure involves imaging before and after $6 \mathrm{D}$ corrections at treatment couch angle. From each pair of successive 6D corrections that were separated by a rotation of the patient, the variation in the prediction of the isocentre was determined. Deviation from rotational invariance was used to infer a confidence limit on stereotactic localisation with respect to the isocentre.
Results From more than 5000 frameless stereotactic radiotherapy observations, the standard error of localisation relative to bony anatomy was determined to be $0.30 \mathrm{~mm}(0.293-0.305 \mathrm{~mm} 95 \%$ confidence interval) in the AP axis. In approximately half of the observation pairs the second data point indicated a subsequent $6 \mathrm{D}$ shift was required.

Conclusions We have augmented our initial phantom based quality assurance of the ExacTrac system by determining the accuracy of the autofusion corrected positioning in clinical practice is comparable to our phantom results. The observed rejection ratio shows that the selected clinical tolerance of $0.7 \mathrm{~mm}$ is near optimal and indicates that the procedure of obtaining the verification images must be retained to achieve this accuracy.

\section{Session 3B-Nuclear medicine and medical imaging}

\section{Photogrammetric tooth surface mapping—a new approach}

\section{Malcolm Grenness ${ }^{1}$ \\ ${ }^{1}$ University of Tasmania}

Recent advances in digital photogrammetry have enabled a new approach to close-range, high resolution mapping of small objects. Recent reports have described the use of consumer grade cameras and materials incorporating optical texture for the mapping of tooth surfaces with precision of around 13 microns. This paper describes a new compact, twin-lens camera system and a new type of optical array for calibration purposes. Improved array target measurement precision and automated measurement has increased the speed and precision of the calibration process. Subsequent imaging and modelling of tooth replicas incorporating optical texture takes around a minute with accuracy in the order of microns and precision in line with previous best results. Examples of dental tooth wear are described and discussed.

Modification of a PET respiratory gating system for amplitude gating

\author{
Robert Barnett ${ }^{1,2}$, Roger Fulton ${ }^{2,1}$ \\ ${ }^{1}$ Sydney West Area Health Service, ${ }^{2}$ Brain and Mind Research \\ Institute, University of Sydney
}

Respiratory gating for PET can be used in motion detection of tumour nodules. Amplitude gating has been suggested for image guided radiotherapy planning, specifically when respiratory gated beam delivery is applied.

Retrospective amplitude gating requires the synchronisation of the measured respiratory trace with the PET list mode stream. This has been achieved by matching time markers which are inserted into both the list mode stream and respiratory trace data set. The temporal accuracy of the time markers depends on the processing and recording of TTL pulses. A correction mechanism for the differing clock rate between scanner and the gating system has demonstrated a deviation of less than 25 milliseconds.

The vendor supplied respiratory gating system for the Siemens Biograph 16 generates TTL pulses at end inspiration in real time, however some cycles were not detected. The system was modified for retrospective gating so that the respiratory trace could be analysed after the scan was completed. Results indicate fewer cycles are rejected because the end inspiration peak detection on the complete trace data set is more robust. 
The amplitude gating scheme was tested on a quality control system which generates a TTL pulse in synchronisation with the sinusoidal movement of a phantom. Amplitude gating using both variably and equally spaced thresholds was compared to phase gating. The comparisons demonstrate the advantages of amplitude gating including less rejected data and less motion blur within frames.

Each gating scheme was compared using clinical PET studies of several oncology cases. The comparison showed the effect of the gating scheme on the detected movement of a single pulmonary nodule.

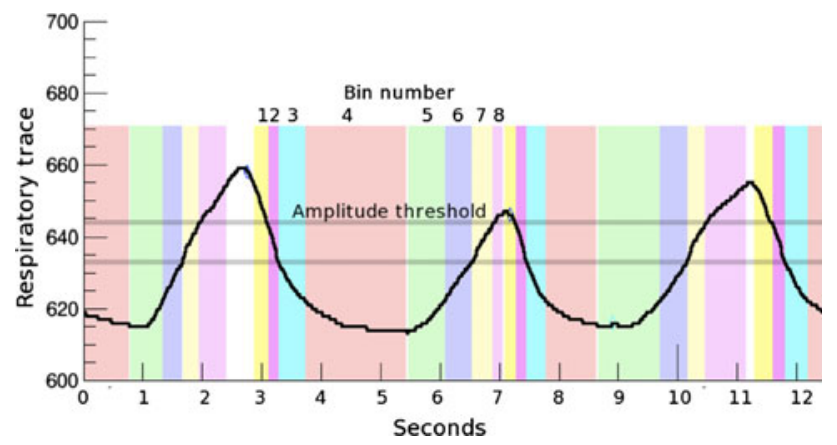

A theoretical comparison between a hybrid emission imager and a collimated anger camera

\section{Jeremy Brown ${ }^{1}$, Matthew Dimmock ${ }^{1}$, John Gillam ${ }^{1}$, Chris Hall ${ }^{1}$ \\ ${ }^{1}$ Monash Centre for Synchrotron Science, School of Physics, Monash University}

The Pixelated Emission Detector for RadiOisotopes (PEDRO) is a hybrid emission imager being developed and constructed at the Monash Centre of Synchrotron Science for small animal Single Photon Emission Imaging (SPEI). PEDRO combines both mechanical collimation (through pinhole apertures or septa) and electronic collimation (through the Compton effect) to decouple the general relationship in SPEI between resolution and sensitivity. A MonteCarlo comparison has been undertaken to compare the performance between PEDRO and a collimated Anger Camera. GEANT4 and GATE simulation packages have been used to conduct simulations in order to accurately compare these two systems. The present work compares the performance of the two imaging systems for $\mathrm{Tc}^{99 \mathrm{~m}}$ $(140 \mathrm{keV}), \mathrm{I}^{131}(364 \mathrm{keV})$ and positron emitters $(511 \mathrm{keV})$ for a number of different geometries.

\section{Development of a software program to assess levels of geometric distortion in MR images for deep brain stimulation procedures}

\section{Tom Greig ${ }^{1}$, Arul Bala ${ }^{2}$, Christopher Lind ${ }^{2}$, Roger Price ${ }^{1}$, Janette Atkinson ${ }^{1}$ \\ ${ }^{1}$ Department of Medical Technology and Physics, ${ }^{2}$ Department of Neurosurgery, Sir Charles Gairdner Hospital}

Deep brain stimulation has traditionally been performed under local anaesthetic with feedback from the patient being integral to the correct placement of the probe inside the brain. However, with the recent developments in medical imaging modalities, the accuracy in locating small targets within the body has improved significantly, which means that surgeons are now performing this surgery under general anaesthetic and are no longer relying on patient feedback for correct placement.
The aim of this project was to develop and test a software program (using MATLAB) that could assess the level of geometric distortion in MR images so that surgeons could have an indication of how reliable their target localisation would be using the BrainLab stereotactic software. To assess the level of geometric distortion, the Leksell ${ }^{\circledR}$ MR Phantom, which contains an axial grid and a coronal grid of regularly spaced rods, was imaged using a similar MR image protocol to the one that was to be used for the patients. These images were then used to compare the actual spacing of the adjacent rods in the axial and coronal slices with that of the measured spacing.

For the surgeons to place the probe in the desired location (posterior subthalamic region) in the patient's brain the MR images of the phantom must exhibit very little distortion and thus, the spacing between adjacent rods in the axial and coronal slices must be within $\pm 1 \mathrm{~mm}$ of the expected distance. Although the first test run of the program showed that it could quantify the discrepancy between the measured distance and actual distance in the $x$ and $y$ directions, it was clear that additional functions needed to be added. That is, post procedure imaging showed that the probe was placed just outside the posterior subthalamic region even though the maximum discrepancy was found to be $0.875 \pm 0.108 \mathrm{~mm}$. Although clinically this was not an issue, it was detrimental to the scientific integrity of the study being performed by Sir Charles Gairdner Hospital Neurosurgeons. From this result, it is believed that the discrepancies might be cumulative, that is, if adjacent rods are consistently distorted in one direction then these individual discrepancies will accumulate and the measured distance between the outer rods will be significantly different to the actual distance. Therefore the program will be revised to include further information regarding the direction of distortion.

\section{Session 3C-Sensor and signal processing}

\section{Modelling of the interaction between intra and extra ocular pressure using electrical circuit elements}

\section{S Mojtaba Golzan ${ }^{1}$, Albert Avolio ${ }^{1}$, Stuart Graham ${ }^{1}$ \\ ${ }^{1}$ Australian School of Advanced Medicine, Macquarie University}

Spontaneous pulsations of retinal vessels have been shown to be associated with changes in cerebrospinal fluid (CSF) pressure behind the eye. Limited measurements of pressure distribution have suggested that an equilibrium is present between pressure in the intra and extra ocular space. However, due to the inherent physical limitations in accessing the ocular space, the characterisation of the interaction of intra and extra ocular pressure still remains unclear. Thus, to investigate ocular and CSF pressure interaction we have developed a model based on measured pressure transfer functions using data from experimental animal studies and configured using electrical circuit elements. The model incorporates description of the extra ocular pressure by including CSF, translaminar and retrolaminar tissue and their relation with the intra ocular pressure including retinal vessel calibre and the systemic blood pressure affecting the intraocular contents directly. The model has been applied to extract an index between the retinal vessel and CSF pressure in which the pressure of the vessels could be estimated based on their diameter changes. We have measured these diameter changes non-invasively using the Dynamic Retinal Vessel Analyzer (Imedos) device. Simulation results show that for constant systemic blood pressure, a decrease in retinal vessel diameter is a consequence of increase in CSF pressure. This study shows that measurement of retinal vessel calibre in association with the electrical circuit model may allow a non-invasive approach to evaluate changes in CSF pressure. 
Central nervous system microstimulation-development of the auditory brainstem implant

Stefan Mauger ${ }^{1,2,3}$, Mohit Shivdasani ${ }^{1,2,3}$, Rebecca Argent ${ }^{2}$, Graeme Rathbone $^{2,3}$, Antonio Paolini ${ }^{1,2}$

${ }^{1}$ Department of Electronic Engineering, La Trobe University, ${ }^{2}$ Bionic Ear Institute, ${ }^{3}$ School of Psychological Sciences, La Trobe University

Auditory brainstem implants (ABIs) are a new neural implant aimed to restore hearing to the profoundly deaf. Currently there have been 800 ABI recipients worldwide who receive poor speech understanding outcomes compared to normal hearing or cochlear implants recipients. Poor performance is thought to be due to many contributing factors including electrode location, stimulation rate and the stimulation strategy. Much research and development is needed to improve ABI recipient speech understanding outcomes.

We therefore systematically investigated responses from single neurons of the auditory midbrain to microstimulation of many locations of the auditory brainstem in anesthetised hooded Wistar rats. Both acoustic and electrical single pulse, pulse train and implant like stimulation were used while recording extracellular and intracellular single neurons responses. Detailed analysis and comparison of acoustic and electrical results has revealed specific neural response characteristics.

Our results show that the auditory system has a specific temporal organisation, some of which is bypassed by electrical stimulation which can be compensated for by stimulation strategies. Our results also found that neural response properties vary with the electrical stimulation rate, and that there may be an optimal stimulation rate for the clinical implant. Inhibition was also found to be prevalent from stimulation of some brainstem locations, indicating both optimal and sub optimal implant locations.

Our animal results provide insight into suitable electrode locations, optimal stimulus rates and advantageous stimulation strategies which are hoped to aid in future ABI developments.

\section{Continuously variable realistic lung gas exchange simulation}

Rebecca Bailey ${ }^{1,2}$, Gavin Robinson ${ }^{1}$, Paul Junor ${ }^{2}$

${ }^{1}$ Alfred Hospital, ${ }^{2}$ La Trobe University

Realistic simulated breath-by-breath gas exchange helps determine the effectiveness of methods of monitoring patient gas uptakes. The simulator is tidally ventilated from a non-rebreathing anaesthetic machine. Our method is modifiable in real-time and demonstrates the effects of the nominated profile of gas uptakes $\left(\mathrm{O}_{2}\right.$, isoflurane and $\mathrm{CO}_{2}$ ). Exchange is simulated using parallel input streams of calculated steady flows into a compliant bellows. Concurrently a parallel output stream of the well-mixed bellows gas is suctioned.

A specified uptake profile corresponds to a parallel input profile and a single suction rate. Accuracy of simulation requires satisfactory measurement of gas exchange. Our simulator responds rapidly to step changes in nominated uptakes and external changes in inspired ventilation and composition.

Bellows exchange: Digital flow controllers (DFC) supply the prescribed inputs of gases which are combined in a manifold before insertion into the bellows while also suctioning well-mixed 'lung' gas. Suction flow control is provided by addition of air by another DFC into a constant suction flow. The DFCs allow step changes to gas flows and are 95\% complete within $1 \mathrm{~s}$.

Overall exchange: Digital flow meters measure fresh and exhaust gas flows of the anaesthetic machine. Rotameter-metered fresh gas flow and composition from the anaesthetic machine can readily be rendered constant and without drift. At the start of simulation fresh gas composition is measured for $5 \mathrm{~min}$. Then exhaust gas flow and composition are continuously measured and 'patient' uptake of each gas calculated.

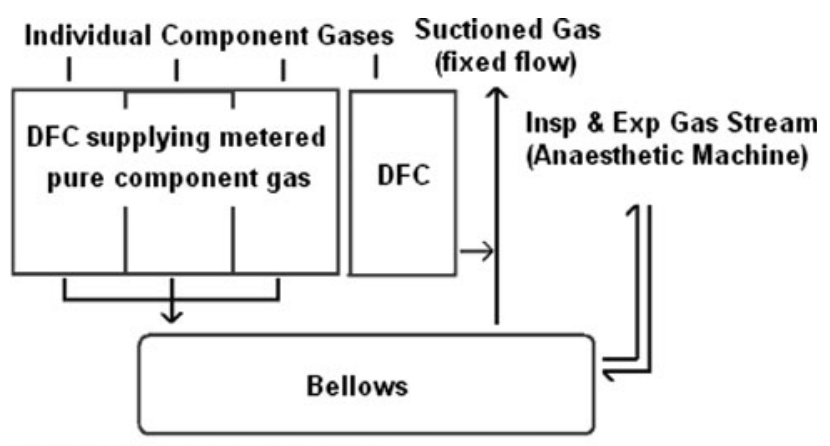

Lung Gas Exchange System

Low-cost electronic health diagnostic equipment for the developing world

Jono Nevile ${ }^{1,2}$, Paul Junor ${ }^{2,1}$, Robert Barnett ${ }^{1,2}$, Julius Yee ${ }^{2,1}$

${ }^{1}$ Alfred Hospital Biomedical Engineering, ${ }^{2}$ Latrobe University Biomedical Engineering

The developed world enjoys good access to electronic diagnostic equipment. The pricing of this equipment doesn't enable the developing world to participate in our modern health care revolution. Lives often appear to be worth less as the equipment that may help to save them is not affordable to these communities. The pricing of equipment for the developed world should support further development. Regulation across the world varies and often limits vendor's ability to provide low cost equipment. Parallel difficulties are seen in what is regarded as basic communication devices in the developed world-PCs. This has been challenged recently by the team at One Laptop Per Child (www.laptop.org). This initiative has opened the possibility for the biomedical engineering community to assist the developing world. Augmenting OLPC rollouts with low cost electronic health diagnostic equipment gives us an opportunity to contribute to our fellow humans. This equipment can be made low cost as it is mostly comprised of simple sensors and sensor systems attached to the laptops. An example of this in the developed world is PC based ECGs. The systems targeted in the first system are: SPO2, ECG and EtCO2. Future systems will include Blood pressure, simple EEG (similar to BIS and Entropy) and temperature. The system developed this year will be presented.

\section{Session 3D-Emergency and disaster medicine}

Development of emergency and disaster management education and research programs and health management programs

\section{GJ FitzGerald ${ }^{1}$}

\section{${ }^{1}$ School of Public Health, Queensland University of Technology}

Recent events have heightened awareness of disaster health issues and the need to prepare the health workforce to plan for and respond to major incidents. This has been reinforced at an international level by the World Association for Disaster and Emergency Medicine which has proposed an international educational framework (1). 
The aim of this paper is to outline recent attempts to develop a national educational framework for disaster health in Australia and to outline the development of educational and research programs in this field.

The framework was developed on the basis of literature and previous experience brought together through a series of workshops and then a modified Delphi technique to finalise the content at each level of the framework and to assign a value to the inclusion of that content at the various levels.

The framework identifies seven educational levels along with educational outcomes for each level. The framework also identifies the recommended contents at each level and assigns a rating of depth for each component. The framework is not intended as a detailed curriculum but rather a guide for educationalists to develop specific programs at each level. This educational framework will provide an infrastructure around which future educational programs in Disaster Health may be designed and delivered and thus permit improved articulation for students between the various levels and greater consistency between programs so that operational responders may be armed with a consistent language and operational approach to the management of major incidents.

The Queensland University of Technology has developed a suite of postgraduate programs that align with this framework and which represent an integrated and coordinated to the delivery of such programs for a range of purposes.

\section{Reference}

Archer F \& Seynaeve G: International Guidelines and Standards for Education and Training to Reduce the Consequences of Events that May Threaten the Health Status of a Community. Prehosp Disast Med 2007;22:120-130.

\section{New battlefield medicine technologies}

\author{
Marc Lakos ${ }^{1}$ \\ ${ }^{1} 1$ st Health Support Battalion
}

The application of new technologies in military health care has decreased the total mortality of soldiers during combat. The lethality of weapons, improvements in acquisition of military intelligence, innovations in command and control and the use of simulation have helped to propel the development and implementation of new technologies.

\section{Australian Defence Force deployable health care}

\section{Tracey Smart ${ }^{1}$}

${ }^{1}$ Royal Australian Air Force

Air Commodore Smart is the senior RAAF medical officer. She will discuss the role of Australian Defence Force (ADF) deployable health care-what it comprises and what it can do, along with some of its challenges. Future health capabilities will also be discussed.

\section{The needs of a mobile first responder-an ambulance perspective}

\section{Howard Wren ${ }^{1}$ \\ ${ }^{1}$ ACT Ambulance Service}

In a relatively short time, technology that was once found only in critical care areas of major hospitals has moved into the pre-hospital patient care environment.
The movement of this technology has been accompanied by many challenges as it is integrated into day to day ambulance practice. Many of these challenges are best met with improved training or modified work practices-however, some lend themselves to a technical solution.

This short presentation will attempt to highlight some of the areas where a technical solution may be possible. It will also address some of the wants as well as the needs of ambulance personnel.

\section{Session 4A-IMRT dosimetry workshop}

\section{Film dosimetry}

\section{Martin Butson ${ }^{1}$ \\ ${ }^{1}$ Illawarra Cancer Care Centre}

Abstract not available at time of printing.

\section{Electronic portal imaging devices as dosimeters for IMRT}

\section{Peter Greer $^{1,2}$}

${ }^{1}$ Calvary Mater Newcastle Hospital, ${ }^{2}$ University of Newcastle

Electronic portal imaging devices (EPIDs) are flat panel amorphous silicon (a-Si) based imagers used in radiation oncology to image patient anatomy before treatment using the treatment beamline. The basic components of the imager are a copper plate for radiation interaction, a scintillating phosphor and an a-Si photodiode array. The non-water equivalence of the materials, energy dependence due to high atomic number composition, optical glare and sensitivity to backscatter make dosimetry with these devices challenging. For planar dosimetry various physical models have been developed to either predict the response of the imager to therapeutic IMRT beams or to convert measured images to water-equivalent measurements. A more recent approach has been to modify the imager design by blocking the optical signal from the phosphor when used for dosimetry. The EPID then acts as a direct-detection device where energy is deposited directly in the photodiode. Models have also been developed to predict the dose deposited within the patient based on EPID measurements. Recently the use of time-resolved EPID dosimetry to obtain dose as a function of gantry angle during volumetric modulated arc therapy has been investigated. This presentation will discuss the dosimetric properties of EPIDs relevant to IMRT, the models and methods used for EPID dosimetry, commercial products available, time-resolved dosimetry with EPIDs and some possible future directions.

\section{Dosimeters in IMRT review}

\section{Brendan Hill ${ }^{1}$, Philip Vial ${ }^{2}$}

${ }^{1}$ Premion, ${ }^{2}$ Liverpool and Macarthur Cancer Therapy Centres

Abstract not available at time of printing.

\section{Session 4B-Monte Carlo}

\section{Monte Carlo model of a X-ray beam for breast radiotherapy dose calculations}

Jung-Ha Kim ${ }^{1}$, Robin Hill ${ }^{1,2}$, Zdenka Kuncic ${ }^{1}$

${ }^{1}$ Institute of Medical Physics, School of Physics, The University of Sydney, ${ }^{2}$ Department of Radiation Oncology, Royal Prince Alfred Hospital 
Introduction The accuracy of the treatment planning system in predicting doses for breast radiotherapy patients is compromised due to limitations in the determination of skin doses, doses in irregular geometries and also in tissue inhomogeneities. This work presents the development of a Monte Carlo model of a linear accelerator for dosimetry calculations of breast radiotherapy patients.

Methods Monte Carlo calculations were performed using the EGSnrc Monte Carlo code (V4 r225) and the BEAMnrc user code. The specifications of the linear accelerator were provided by the manufacturer. Optimisation of the incident X-ray beam was achieved by an iterative adjustment of the energy, spatial distribution and radial spread of the incident electron beam striking the target and optimisation of the flattening filter [1]. Energy cutoff parameters were PCUT $=0.01 \mathrm{MeV}$ and ECUT $=0.700 \mathrm{MeV}$. Directional bremsstrahlung splitting was switched on for all BEAMnrc calculations to ensure calculation efficiency.

The measurement data set consisted of depth dose and beam profiles from the $6 \mathrm{MV}$ X-ray beam generated by a Varian Clinac 21iXs (Varian Medical Systems, Palo Alto, USA) linear accelerator in Royal Prince Alfred Hospital. This data was for a range of field sizes ranging from $5 \times 5$ to $30 \times 30 \mathrm{~cm}^{2}$.

Results and discussion The energy of the incident electron beam was varied by up to $1.0 \mathrm{MeV}$ from the nominal beam energy of $6.0 \mathrm{MeV}$. The closest agreement for depth doses, to within $2 \%$, occurred with an incident electron energy of $6.5 \mathrm{MeV}$. Optimisation of the calculated beam profiles was more difficult for larger field sizes (at least $20 \times 20$ ), with the optimal electron radial spread requiring significant comparisons until the optimal value of beam radius was found.

Conclusions We have developed a Monte Carlo model of a $6 \mathrm{MV}$ $\mathrm{X}$-ray beam and further development will be undertaken to include MLCs in the X-ray beams.

Acknowledgments The authors acknowledge the financial support of the National Breast Cancer Foundation.

\section{Monte Carlo dosimetry for stereotactic radiosurgery}

Tanya Kairn ${ }^{1}$, John Kenny ${ }^{2}$, Jamie Trapp ${ }^{1}$

${ }^{1}$ School of Physical and Chemical Sciences, Queensland University of Technology, ${ }^{2}$ Premion, The Wesley Hospital

Stereotactic radiosurgery (SRS) treatments for brain cancers require small and precisely shaped photon beams. These beams can be generated by fitting a linear accelerator with a micro-multileaf collimator (mMLC) such as the BrainLAB m3, which offers greater flexibility for field shaping than standard SRS cone collimators. The use of such a novel technology to treat such a highly radiosensitive organ demands rigorous prior analysis and verification. However, the accuracy of treatment planning dosimetry is difficult to verify by measurement alone due to the physical limitations of the measuring devices available. There is therefore an obvious advantage to being able to simulate mMLC treatments a using well-known and clinically verified Monte Carlo code, such as BEAMnrc.

Due to the tri-faceted shape of the BrainLAB m3 mMLC's leaf ends, however, it has been assumed that the component modules in the standard BEAMnrc distribution are insufficient to model this device. This study contends, however, that geometrically and dosimetrically accurate Monte Carlo simulations of radiotherapy beams collimated by the BrainLAB $\mathrm{m} 3$ can be completed using BEAMnrc's standard component modules.

This contention is tested by simulating dose distributions in water and comparing these with clinical film and ion chamber measurements and thereby:
- verifying the geometric accuracy of the simulation parameters for modelling the mMLC leaf sides, through comparison of real and virtual film images of closed leaves (Fig. 1a) and individually closed leaves (Fig. 1b);

- verifying the geometric accuracy of the simulation parameters for modelling the mMLC leaf ends, through comparison of real and virtual film images of complex fields (Fig. 1c-f);

- verifying the (relative) dosimetric accuracy of the mMLC (and linac) model, through comparison of real and virtual water tank depth-dose and lateral profiles;

- verifying the accuracy of the mMLC (and linac) model (especially the definitions of the materials from which it is made), through comparison of scatter factors calculated from real and virtual water tank data.
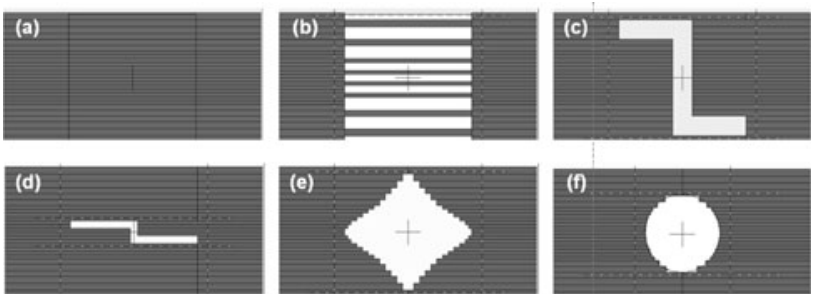

Fig. 1

Importantly, the model mMLC produced through this work is based on physical measurement and published data, rather than confidential information obtained from the manufacturer. This fact, combined with the utilisation of only standard BEAMnrc component modules, means that the major outcome of this study is the production of a model mMLC that can be shared and used by medical physicists nationally and internationally.

Acknowledgments This work is funded by the Wesley Research Institute. Computational resources and services used in this work were provided by the HPC and Research Support Group, Queensland University of Technology, Brisbane, Australia.

\section{Monte Carlo modelling and experimental characterisation of the MOSkin radiation dosimeter at clinical kilo voltage energies}

Cheryl Lian ${ }^{1}$, Mohd Amir Radhi Othman ${ }^{1}$, Dean Cutajar ${ }^{1}$, Martin Butson ${ }^{2}$, Susanna Guatelli ${ }^{1}$, Anatoly Rosenfeld ${ }^{1}$

${ }^{1}$ Wollongong University, ${ }^{2}$ Illawarra Cancer Care Centre

Introduction The widespread use of modern helical CT scanners poses new challenges in radiological protection and dose monitoring. An ideal radiation dosimeter for diagnostic radiology should be tissue equivalent and have a flat energy response across a broad range of energies. Development of a reliable real-time dosimeter for skin dosimetry for kilovoltage energy photons at relatively low doses is an issue due to the requirement for tissue equivalence. The new MOSkin detector is a strong candidate for this application.

Objectives The aim of this study was to examine how the dose response of the MOSkin varies with energy, for irradiations on phantom surface and at various depths in phantom.

Materials and method A Monte Carlo model of the MOSkin dosimeter was constructed using the GEANT4 Toolkit and its dose response simulated for monoenergetic photon beams from 15 to $300 \mathrm{keV}$ and spectral photon beams in the range $50-250 \mathrm{kVp}$. The energy response factors obtained by the Monte Carlo simulations were subsequently verified by MOSkin measurements on the Gulmay D3300 orthovoltage machine. 
Results A close match was obtained between experimentally determined energy response factors and Monte Carlo derived energy response factors for the MOSkin radiation dosimeter.

Conclusion GEANT4 is a suitable tool for simulating dosimeter energy response. Through the judicious selection of appropriate energy filters, it is now possible to design appropriate packaging to further optimise the energy response of the MOSkin dosimeter.

\section{Development of a Monte Carlo model for CVD diamond detectors in radiotherapy}

Florentina Baluti ${ }^{1}$, Juergen Meyer ${ }^{2}$, Hossain Deloar ${ }^{1}$, Stuart Lansley ${ }^{2}$, Greg Betzel ${ }^{2}$

${ }^{1}$ Radiation Oncology, Christchurch Hospital, New Zealand, ${ }^{2}$ Department of Physics and Astronomy, University of Canterbury, New Zealand

Introduction Two methods have been developed for estimating dosimetric errors created by inaccuracies in MLC leaf positions for the sliding window IMRT

Methods In the first pre-treatment method, a two dimensional map is generated with values of expected dosimetric error for a given assumption of an average leaf gap width uncertainty. Estimation of dosimetric errors is based on a correlation of dosimetric error with a gap width and a width uncertainty of this gap. The second posttreatment method uses dynamic log files generated by the MLC controller after executing the IMRT treatment plan. Those files are first converted into MLC files by in-house developed software. Then using a treatment planning system (TPS-Eclipse ${ }^{\circledR}$ ), original MLC files of an IMRT treatment plan can be substituted with converted dynamic $\log$ files and the dose delivered to the patient can be recalculated.

Results In the pre-treatment method, it was found that significant dosimetric errors occurred at sharp changes of gradients in dose profile. In the post-treatment method, a mean dose delivered to the CTV had differed on average by $-1.5 \%$. This relatively large discrepancy was further investigated. Importing MLC files from the TPS and exporting them back unchanged caused the TPS to miscalculate doses delivered to the CTV on average by $-1.6 \%$. After correction for this error, the post-treatment method shows average dose difference of $0.1 \%$.

Discussion and conclusion Two MLC error analysis methods show relatively quick and easy techniques of estimating dosimetric errors caused by inaccuracies of MLC leaf positions.

\section{Session $4 \mathrm{C}-$ Technology in the operating room}

\section{Medical equipment in operating theatres}

\section{Mike $\mathrm{He}^{1}$ \\ ${ }^{1}$ Calvary Hospital}

With the advances of medical technologies, more and more medical equipment are used in operating theatres. Good, reliable and innovative products improve surgical outcomes and enhance the quality of patients' care. To understand the applications and advantages/disadvantages of the surgical equipment are so important for both surgeons and medical technologists. This presentation here is to introduce some of the equipment used in operating theatres and to discuss the applications of these.
Using heart rate variability to monitor fentanyl-induced changes in ANS preceding respiratory depression

Anne-Louise Smith ${ }^{1,2,3}$, Harry Owen ${ }^{2}$, Karen Reynolds ${ }^{3}$

${ }^{1}$ Flinders Biomedical Engineering, Flinders Medical Centre, ${ }^{2}$ School of Medicine, Flinders University, ${ }^{3}$ School of Informatics and Engineering, Flinders University

Opioids have an occasional but high risk side effect of respiratory depression. Respiratory depression covers all respiratory events from a decrease in respiratory rate $<10$ breaths/min or $\mathrm{SpO} 2<90$, to central or obstructive apnoea with many brief and insignificant episodes as the opioid effect on respiration waxes and wanes. The detection of critical respiratory depression is done after the event. Earlier detection would be beneficial in preventing increased morbidity and mortality of $0.01 \%$ patients receiving analgesic opioids.

Fentanyl induced changes in the autonomic nervous systems (ANS) have been detected by heart rate variability (HRV). The major effect of opioids is directly on the central nervous system with decreases in sympathetic activity, reduction in parasympathetic activity and vagal tone. Sleep apnoea is also known to produce changes in the HRV.

It is unknown (a) if changes to the ANS precede critical respiratory depression, and (b) if these can be detected with HRV.

HRV indices are usually used over $5 \mathrm{~min}, 15 \mathrm{~min}$ and $24 \mathrm{~h}$ periods. This study requires a selection of HRV indices that work on nonstationary signals, over sub-minute periods.

Methods This pilot observational study investigated the use of HRV indices to detect changes in the ANS preceding respiratory depression in patients pre-operatively administered with fentanyl. HRV indices were selected for (a) sensitivity to respiratory modulation and vagal tone and (b) ability to register sub-minute (ultra short term) dynamics. Results Preceding respiratory depression, few changes to the ANS could be detected by sub-minute HRV analysis.

Discussion Fentanyl causes respiratory depression through modulation of the ANS respiratory centres however very sub-minute measures of HRV are not sensitive enough to these modulations to detect respiratory depression.

Anaesthesia and consciousness depth monitoring correlates and design

\section{Burton ${ }^{1,3}$, PS Myles ${ }^{2}$, I Brown ${ }^{1}, \mathrm{M} \mathrm{Xu}^{3}$, E Zilberg ${ }^{3}$ \\ ${ }^{1}$ Department of Electrical and Computer Systems Engineering, Biomedical Engineering, Monash University, ${ }^{2}$ Department of Anaesthesia, Alfred Hospital, ${ }^{3}$ Compumedics Ltd}

Introduction The feasibility and performance outcomes of an experimental anaesthesia and consciousness depth (A\&CD) hybrid (AEP and EEG) monitoring system were established. AEP, EEG, $\mathrm{BIS}^{\mathrm{TM}}$ index, and clinical consciousness observations were simultaneously recorded from 20 anaesthetised surgical patients. Consciousness prediction accuracy, online event detection sensitivity, and responsiveness to consciousness transitions were evaluated.

Results Significant $(P<0.05)$ values were derived (pat. $n=16)$ for latency intervals for the differential-amplitude (AEPDA) index (i) method, and for most of the power-amplitude (AEPPD) latencyintervals. Overall the $P$-values for AEPDA were much lower than for the AEPPD method. The consciousness prediction performance of BIS $^{\mathrm{TM}}$ and 64 AEP analysis methods were examined, including new non-linear dynamic (entropy), slow moving time average (MTA; 256-sweep; $38 \mathrm{~s}$ response), fast MTA (autoregression-AEP (arx) 15-sweep; 2.2. s response) and latency-interval dependent values. The best AEP fast MTA values (pat. $n=4$ ) were found to be 
EntropyAEPiDA[arx15;0-140 ms] with estimated area under the receiver operating characteristic curve (AROC) outcomes of 0.9778 . The best AEP slow MTA outcomes (pat. $n=4$ ) were found to be AEPiDAS[ave256;0-140 ms], AEPiDAS[ave256;80-140 ms], followed by EntropyAEPiDA[ave256;80-140 ms] values, with AROC outcomes of $0.9792,0.9789$, and 0.9784 , respectively. BIS ${ }^{\mathrm{TM}}$ index demonstrated the best consciousness prediction AROC of 0.9979 . BIS $^{\mathrm{TM}}$ response to consciousness transition was found to be $60 \mathrm{~s}$ slower than the fast MTA and 38 s slower than slow MTA.

Conclusion While the practical constraints of AEP-based anaesthesia monitoring cannot be overlooked, individual AEP latency-intervals, particularly those related to new entropy and PAMR measures, together with differential analysis (AEPiDAS), were shown to be promising predictors of consciousness.

\section{Session 4D—Biomedical engineering}

\section{Device requirements of intermittent photic stimulation for photosensitive epileptic patients}

C Glanville ${ }^{1}$, P Junor ${ }^{1}$

${ }^{1}$ Department of Electronics Engineering, La Trobe University

Intermittent photic stimulation involves using light of a given frequency, intensity and wavelength, to assess individual patients' susceptibilities to light stimulus. This assists diagnosis of epilepsy and aids patient management strategies in avoiding stimulating seizures by determining the types of stimulation (i.e. wavelength, intensity and frequency) to which the patient is most sensitive.

This paper looks into those characteristics which are optimal for eliciting EEG responses, and we investigated photic stimulation requirements from an engineering perspective. Survey of the literature determined that neither the biggest nor brightest light is necessarily the most effective: rather a more considered approach would improve its success at eliciting responses.

The study was prompted by commercial interest, where performance is the major promoting factor in such devices. However power consumption is an important consideration in real devices, motivating research into the minimum practical flash/on periods. A time-constant approach was taken to find the minimum on-time required to produce adequate photocell receptor potential changes. We determined that on-time can be significantly reduced (to a limit determine by the characteristics of the photocells) without affecting performance, thus enabling reduced power consumption.

Other factors found to be relevant include the wavelength of stimulation and the field of view coverage, which, in combination with the signal processing performed by the cells within the eye, play a major role in the performance of strobe devices. These findings now inform the possible modification of photic stimulation devices.

\section{Athlete video tracking system}

Andrew Walsh ${ }^{1}$, Bruce Satchwell ${ }^{2}$, Kim Barnett ${ }^{2}$, Justin Grantham ${ }^{3}$

${ }^{1}$ Macquarie University, ${ }^{2}$ Alive Technologies Pty Ltd, ${ }^{3}$ ASPETAR, Qatar Orthopaedic and Sports Medicine Hospital, Qatar

Athlete positional tracking is useful as a coaching aid but also can be used to estimate subject distance/velocity/acceleration profiles and energy expenditure. Such systems generally fall into two techniques, GPS tracking or video (camera) based. The former require the athletes carry GPS trackers with them during the event and do not work indoors or in stadiums surrounded by high spectator stands. GPS positional accuracy may also be inadequate for some sports (e.g. basketball, tennis).

Camera-based systems have potentially better accuracy and may also work indoors. However, development times tend to be long due to the complex nature of the task. RoboRealm ${ }^{\mathrm{TM}}$ software was used to develop a video processing application that tracks athletes and maps their motion onto a two dimensional map of the field. MATLAB ${ }^{\mathrm{TM}}$ was used to calibrate a non-linear two dimensional transform to change the coordinates from the video pixels $(\mathrm{xv}, \mathrm{yv})$ to rectangular coordinates $(\mathrm{xm}, \mathrm{ym})$ and a JAVA program was written to further display the player movements in real time on a 2D map. With further development, such a system could be used as a coaching aid in the various football codes or athletics. RoboRealm ${ }^{\mathrm{TM}}$ facilitates rapid prototyping of such visual processing algorithms.

Flow metabolism coupling as an indicator of depth of anaesthesia

Thushara Perera ${ }^{1}$, Phil Lewis ${ }^{2}$, Paul Junor ${ }^{1}$

${ }^{1}$ Department of Electronic Engineering, La Trobe University, ${ }^{2}$ Department of Neurosurgery, The Alfred Hospital

The hemodynamic state of the brain (as with many other organs) is, under normal circumstances, directly related to activity. To match metabolic requirements to energy delivery, local brain tissue perfusion is carefully regulated by vasodilation/constriction mechanisms. This physiological action gives rise to flow-metabolism coupling (FMC) and to the possibility of depth-of-anaesthesia monitoring in a healthy brain. FMC can be used as an indicator of brain activity, from which the level of consciousness may be inferred. Our initial goal is to develop a method of monitoring FMC and subsequently correlate it with depth of anaesthesia.

Functional near-infrared (fNIR) neuroimaging can be used to accurately monitor focal cortical tissue blood-volume and oxygen saturation. This appears to be a suitable FMC monitoring technique because fNIR devices can be produced at low-cost and are noninvasive. The problem of motion artefact can be reduced by signal gating and secure probe attachment, but inter-patient variations remain a difficult issue.

To improve the specificity of the monitoring system and obviate the need to account for extracranial contamination, a visual stimulus (flashing light) will be presented to the patient. Theoretically this will enable the measurement of a visually-evoked FMC response localised near the occipital lobe. During anaesthesia, the stimulus must be of significant intensity to penetrate closed eye lids.

Intellectual property management as a part of technology management for medical device technologies

Maryam Khajeh ${ }^{1}$, Alistair Smith ${ }^{1}$

${ }^{1}$ Davies Collison Cave

A good intellectual property (IP) management plan can provide significant economic and commercial benefits, particularly when implemented in parallel with biomedical device research and development. This discussion aims to highlight some of the major steps involved in developing and maintaining an effective IP management plan for biomedical devices, and how this can assist in realising commercial outcomes.

The IP management plan steps can include performing literature and patent searching, both at the onset of a research proposal and continuously throughout research and development, to thereby identify areas in which product development is both required and commercially viable. 
During the research and development process, potential types of IP which reside in a developed device should be identified, with steps being taken to protect the IP taking into account device development, clinical trials, jurisdictional restrictions, funding, and publication requirements. Throughout this process, documentation should be established in order to provide evidence regarding product development and inventor contributions.

The process should also include consideration of the intended business plan, with a view to leveraging the IP to achieve a successful commercial outcome.

\section{Session 5A-Imaging in radiotherapy}

\section{Three-dimensional analysis with daily stereoscopic X-ray imaging of intrafraction patient motion in head and neck treatments using five points fixation masks}

\author{
N Senthilkumar ${ }^{1}$, Suresh Rao ${ }^{1}$, Lisa Jose ${ }^{1}$ \\ ${ }^{1}$ Department of Oncology, Father Muller Medical College, India
}

Objective Image-guided radiation therapy (IGRT) plays an important role in the delivery of intensity-modulated radiotherapy (IMRT) and linear accelerator (LINAC)-based stereotactic radiosurgery because of the requirement by those techniques for precise localisation of the target. Our aim in this study was to determine the intrafraction motion of patients immobilised with a five-point thermoplastic mask for head and neck treatments. For this study seven patients were consecutively assigned to use a five-point thermoplastic mask to determine the intrafraction motion.

Materials and methods The Novalis IGRT system consists primarily of two floor-mounted kilovoltage X-ray tubes that project obliquely from lateral to medial, posterior to anterior, and superior to inferior onto two corresponding flat-panel detectors mounted on the ceiling and an infrared external marker monitoring subsystem. The objectives of the infrared subsystem are twofold to perform the initial patient setup according to external skin markers, and to control the patient and couch positions with superior accuracy once position deviation is determined by image fusion. The patients included in this study have been treated with DMLC IMRT. Patient positioning is based on the IR reflecting marker detection by ExacTrac BrainLAB that is capable of real-time monitoring and adjustment of the patient setup. The IR markers, composed of a socket and a sphere that fits into the socket, are placed on marked spots on the patient's skin or on the immobilisation device. After initial patient setup with the infrared subsystem, two X-ray images from the pair of flat-panel detectors are acquired and fused with the 3D CT simulation images. The auto-fusion software provides fusion algorithms gives three degrees of freedom called 3D fusion. Specifically, the software compares the two X-ray images with corresponding DRRs calculated from the CT images with various translational shifts and finds the pair of DRRs with maximal similarity to the X-ray images to be the best match.

Results and discussion The number of treatment fractions per patients treatment course ranged from 21 to 33 fractions (mean 26.8 fractions) and total amount of 188 treatment fractions could be used for the evaluation of the intrafraction patient motion. The intrafraction motions were evaluated the lateral, longitudinal, vertical shifts $(\mathrm{mm})$ mean (SD; range) were First initial setup correction $2.7(1.43 ; 4.88)$, $2.43(0.44 ; 5.5), 3.4(2.05 ; 5.16)$, before X-ray correction $1.14(0.8$; $2.46), 1.277(0.3 ; 2.49), 1.388(0.6 ; 1.86)$ and after X-ray correction shifts were 0.319 (0.177; 0.5), $0.398(0.28 ; 0.537), 0.476(0.2 ; 0.83)$. Conclusion From the values obtained it is concluded that we can achieve less than $1 \mathrm{~mm}$ in the treatment delivery in IMRT with the help Exact trac-IGRT system.
Simple and efficient pre-treatment IMRT verification using EPIDs

Philip Vial $^{1,2}$, Shrikant Deshpande ${ }^{1}$, Sankar Arumugam ${ }^{1}$, Gary Goozee ${ }^{1}$, Lois Holloway ${ }^{1,2,3}$

${ }^{1}$ Department of Medical Physics, Liverpool And Macarthur Cancer Therapy Centres, ${ }^{2}$ Institute of Medical Physics, School of Physics, University of Sydney, ${ }^{3}$ Centre for Medical Radiation Physics, University of Wollongong

Purpose To investigate an efficient method of verifying intensity modulated radiation therapy (IMRT) treatments using electronic portal imaging devices (EPID).

Methods and materials Normalised profiles of open beam EPID images and reference dose in water were compared for different setup parameters to determine the closest match. Gain corrected EPID data was collected for Elekta and Siemens linacs with 0, 5, and $10 \mathrm{~cm}$ of solid water build up added directly on top of the EPID. Reference dose in water profiles were collected at $1.5,5$, and $10 \mathrm{~cm}$ depths. Offaxis ratio and penumbra width was compared. The most accurate and efficient configuration was trialled for clinical IMRT quality assurance (QA).

Results and discussion The best results were obtained using EPID images acquired without additional build-up and dose in water data at $10 \mathrm{~cm}$ depth. Using this configuration the agreement in off-axis ratios was within 1 and $2 \%$ for the Elekta and Siemens EPIDs, respectively. The agreement in penumbra width (80-20 distance) was within $1.5 \mathrm{~mm}$ for both EPIDs. This was also the most efficient method to set-up. Having no build-up permits the use of treatment gantry angles. In preliminary IMRT studies $94 \%$ of pixels passed a $3 \% / 3 \mathrm{~mm}$ gamma criteria. Automated analysis methods are being investigated to further reduce QA time. Time studies are required to quantify efficiency gains. This method could be used for any combination of vendor equipment.

Conclusion A simple and efficient method for pre-treatment IMRT verification using EPIDs was determined.

\section{Amorphous silicon EPID application for routine quality} control and IMRT verification

\section{Tania Groudeva ${ }^{1}$, Keith Croft $^{1}$}

${ }^{1}$ Department of Radiation Oncology, Palmerston North Hospital, New Zealand

Introduction Dose-response characteristics of an amorphous silicon electronic portal imaging device (EPID) were investigated. Its suitability as a check device for monitoring LINAC beam parameters as well as for IMRT verification was evaluated.

Methods All measurements were done using OPTIVUE EPID mounted on Oncor medical accelerator (Siemens Medical Solutions Inc.) with $6 \mathrm{MV}$ photon energy and source to imager distance of $115 \mathrm{~cm}$.

A method for IMRT verification using EPID was created by designing a phantom in the $\mathrm{XiO}$ planning system containing objects with different electron density and establishing a proper EPID calibration factor. The purpose of it was to model the EPID response given after applying uniformity, dead pixel and offset corrections. In order to confirm the ability of our IMRT verification procedure to detect accurately positional and dosimetrical differences certain alterations of an original IMRT plan were introduced. Then the gamma-function of the original and modified $\mathrm{XiO}$ treatment plan was compared with the gamma function of the original $\mathrm{XiO}$ and delivered to EPID modified plan. 
Results Good dose-response linearity was found. LINAC parameters as dose output, filed size and shape measured with EPID agreed very well with these received using our current measuring device. A pattern for testing MLC leaves position and dose reproducibility was created. Relative changes in these parameters are monitored using gamma-function analysis.

The accuracy and sensitivity of the IMRT verification method was confirmed to be very good.

Discussion and conclusions The EPID geometrical stability and reproducibility of the results show that it can be very useful tool for routine quality control, including measuring of dose output, field size and shape, virtual wedge profiles and MLC leaves position check. The results show that the EPID IMRT verification procedure we developed can be confidently used for verifying the accuracy of IMRT delivery.

Cone beam CT as a tool to verify the internal target volume for lung cancer radiotherapy

Tomas Kron ${ }^{1,2}$, Natalie Clements ${ }^{1}$, Leon Dunn ${ }^{2}$, Julie Miller ${ }^{1}$, Aldo Rolfo ${ }^{1}$, David Ball ${ }^{1}$

${ }^{1}$ Peter MacCallum Cancer Centre, ${ }^{2}$ RMIT University, Melbourne

Background and aim Daily image guidance has greatly improved our ability to determine target position for external beam radiotherapy. However, in the case of moving targets it is often necessary to verify also the extent of motion of the tumour, usually referred to as internal target volume (ITV). It was the premise of the present work that slow imaging such as Cone Beam CT (CBCT) should be able to acquire aspects of the target in all locations during the breathing cycle thereby providing an image of the ITV, albeit blurred.

Methods Different targets (graphite in lung equivalent material, air cavities in Perspex) were fitted in a moving phantom (Quasar, Modus Medical) and imaged using Varian CBCT on a Trilogy linear accelerator (half fan, $60 \mathrm{~s}$ rotation, 660 projections, $125 \mathrm{kV}$, $40 \mathrm{~mA}$ ). Different sinusoidal moving patterns were studied and the extent of the visible target assessed in coronal and sagittal reconstructions.

Results Compared to a stationary object, the track of a moving target can be clearly seen in CBCT. For high contrast objects of sizes between 5 and $20 \mathrm{~mm}$ with movement between 20 and $40 \mathrm{~mm}$ the extent of motion was on average $3 \mathrm{~mm}$ shorter than the actual motion and could be clearly determined up to motion periods less than $1 / 8$ th of the CBCT acquisition time ( $8 \mathrm{~s}$ period). For longer periods and objects of less contrast the determination of the ITV becomes less accurate.

Conclusion CBCT is capable of depicting the ITV of high contrast objects for typical patterns of patient breathing in radiotherapy.

\section{Session 5B-Medical imaging}

\section{DRL survey}

Abstract not available at time of printing.

\section{Topic group: radiology special interest discussion}

Abstract not available at time of printing.

\section{Session 5C-Asset planning and management}

\section{Asset Management Program 2009—asset management risk} assessment matrix

\section{Adrian Scott ${ }^{1}$, Martin Van Lith ${ }^{1}$, Melanie Brown ${ }^{1}$ \\ ${ }^{1}$ ACT Health}

Background A key component the Strategic Asset Management planning process is the risk assessment of assetable medical and nonmedical equipment.

The format previously used by ACT Health for risk assessment of assetable equipment no longer complied with the current standard (AS/NZS 4360:2004), which prompted the development of the new Risk Assessment Matrix.

The new format comprises the main elements of Consequence, Impact, Likelihood and Condition and presents these in a streamlined matrix which also includes a Recommendation.

This new format complies with the current standard and meets ACT Health's requirements from an Accreditation perspective.

Aims The purpose of risk assessing assetable equipment is to provide a guideline for when equipment should be replaced.

Methods

- A trial of the draft matrix was undertaken which found that the new matrix is easy for staff to understand and use and also accurately reflects what staff feel to be the risk rating.

- Received Portfolio Executive endorsement.

Outcomes The new matrix was used successfully across all areas of ACT Health as a key component of the Stock Take 08/09 project. This has ensured that ACT Health assetable equipment has now been appropriately risk assessed. It has proven to be a highly successful communicative tool and provided Asset Management with accurate information regarding the current condition of equipment with in ACT Health. It has also provided a solid foundation for Replacement Request submissions to the P\&E Committee and allowed a system for effective prioritisation of requests.

\section{Asset management workshop}

Melanie Brown ${ }^{1}$, Phil Thorburn ${ }^{2}$, Ken Wilson ${ }^{3}$, Willy Vandenberg ${ }^{4}$, Helen Riddell ${ }^{5}$

${ }^{1}$ ACT Health, ${ }^{2}$ Royal Adelaide Hospital, ${ }^{3}$ BEHTS, ${ }^{4}$ NSW Department of Commerce, ${ }^{5}$ DHS Victoria

The asset management workshop has been introduced to the conference to provide an up date on how states and territories plan for effective management and replacement of medical equipment.

Whilst most biomedical engineers are involved with local asset management, this workshop will focus on the 'bigger picture'. Presentations will be given by key government personnel from participating states on how medical equipment can be managed across the state or territory.

The workshop will provide up to date information on:

- asset management techniques.

- promoting asset management in hospitals.

- becoming pro-active in asset management programs.

- sharing experiences and ideas.

- getting the right information to government planning staff.

Supporting state and territory planning means better equipment outcomes for your hospital. 


\section{Session 5D—Neonatal and paediatric medicine}

\section{Neonates and paediatric medicine}

\section{Zsuzsoka Kecskes $^{1}$}

${ }^{1}$ The Canberra Hospital

Neonates and paediatric medicine differs from adult. Tight tolerances and special care is the key to improve patient's survival rate. Medical technology plays a crucial role in neonate medicine to achieve these goals.

This presentation will elaborate/discuss the following:

- What is current practice in neonates?

- How the current technology assists health care workers to care remotely?

- The current status of technology used in neonate medicine.

- The future technology needs in this area of medicine.

\section{Innovations to a unique, free-field, sound effects audiometer}

\section{Thushara Perera ${ }^{1,2}$, Peter Taunton ${ }^{2}$, Christine Poulis ${ }^{3}$}

${ }^{1}$ Department of Electronic Engineering, La Trobe University, ${ }^{2}$ Department of Biomedical Engineering, The Royal Children's Hospital, ${ }^{3}$ Department of Audiology, Royal Children's Hospital

StimLab, a distinctive free-field audiometer, was designed and developed at the Royal Children's Hospital in the late 1980s. ${ }^{1}$ Over the two decades of operation it has been effective in paediatric hearing loss examinations. Whereas other audiometers relied solely on pure or warble tones, StimLab used common sound-effects as stimuli. This design feature remains unique to-date.

The existing StimLab hardware is ageing and difficult to support. It uses a $30 \mathrm{MHz}$ computer running MS-DOS, three external digital signal processors, an audio amplifier, two speakers and two visual reenforcement puppets. The hardware is beginning to fail, therefore a complete redevelopment was deemed necessary.

The new version utilises a $1.8 \mathrm{GHz}$ notebook computer capable of software-based signal processing. The key goals of initial development were to: (1) integrate all functionality into a standard PC, (2) implement real-time audio filters and (3) automate the exhaustive calibration process.

Dependency on external signal processors was eliminated by interfacing to the PC sound card using a third-party software library. ${ }^{2}$ Infinite-impulse response filters were designed in Matlab ${ }^{\mathrm{TM}}$, then transcoded into $\mathrm{C}++$ for real-time audio filtering. Finally, an automatic algorithm was developed to determine the system calibration curves using an external sound level meter. The advantages of StimLab over conventional free-field audiometry will be investigated after thorough testing of its extended functionality.

\section{References}

1. R. Newman, 'StimLab User Manual,' Royal Children's Hospital, Melbourne, Australia, Tech. Rep, 1992.

2. Un4Seen Developments, BASS: audio library. [C++ API]. Available: http://www.un4seen.com/ [Accessed: Mar. 24, 2008].

\section{Pulse oximeter sensor accuracy and patient safety}

Geoff Mathews ${ }^{1,2}$, Veronica Hickson ${ }^{1,2,3}$

${ }^{1}$ The Electrode Company Ltd, ${ }^{2}$ Endovations, ${ }^{3}$ Gwent Health NHS Trust, Wales
Pulse oximetry is influential in the management of oxygen therapy. There is a problem with pulse oximeter accuracy due to variations in sensor accuracy.

The SATs value displayed on the monitor is dependent on the oxygenation state of the haemoglobin and the wavelengths of the light emitted by the sensor. It is assumed that wavelengths are fixed and the only variable is the haemoglobin oxygenation. This is not always true, and variations in wavelengths result in erroneous SATs readings. Any error associated with the sensor gets greater at lower SATs.

High reading sensors can lead to oxygen therapy being deferred or not given at all with the obvious impact on morbidity and mortality. Low reading sensors can lead to excessive oxygen therapy - a causative factor in ROP, and a problem where a depressed respiratory drive leads to $\mathrm{CO}_{2}$ retention and metabolic disturbances. Low reading sensors can also contribute to unnecessary and potentially harmful interventions such as intubation.

Sensor accuracy can be calculated from knowledge of a sensor's spectral properties. Sensors can be inaccurate from new or as a result of ageing. A survey of 1500 sensors revealed that $30 \%$ were unacceptable.

There are sensors in use where if clinical decisions are made on the data produced then the patient could get the wrong treatment. Patient safety is improved by removing unacceptable sensors from use. Increasing user confidence in pulse oximeter equipment leads to better management of oxygen therapy, following of guidelines and improved patient outcomes.

\section{Session 6A-Management of technology in radiotherapy}

\section{Paperless medical physics QA in radiation therapy}

Jianjie Luo ${ }^{1}$, Sean White $^{1}$, Shan Yau ${ }^{1}$

${ }^{1}$ Nepean Cancer Care Centre, Nepean Hospital

Introduction Physics QA is an integral part of a medical physicists role in cancer care. Management of physics QA documents is time consuming. Storage space, archive administration (the search for old results) and paper consumption are just some of the difficulties faced by physicists. Plotting trends and drawing meaningful conclusions from these results can be challenging using traditional QA methods. Remote checking of QA within a hospital network can also be problematic. The aim of this project is introduce a paperless QA system that will provide solutions to many of these issues.

Method The introduction of a paperless QA system required hardware and software to be installed at our centre. A computer acting as a server was set up in the existing hospital computer network environment. This server acts as a database for all QA results. Microsoft Access and IIS (Internet Server) were installed on this server. ASP (Active Service Page) with VBScript and JavaScript technique were used as foreground applications to provide access to the database through the network. An SQL search engine was employed on the server to assist in the sorting and graphing of required results. International, national and local protocols, e.g. IEC, ACPSEM, AAPM and NCCC were used to define the tolerance levels for QA. In vivo flash charts were applied to graph trends according to user requirements. All the client computers through the network can visit this password-protected database using Internet Explorer. 


\section{Results and conclusion}

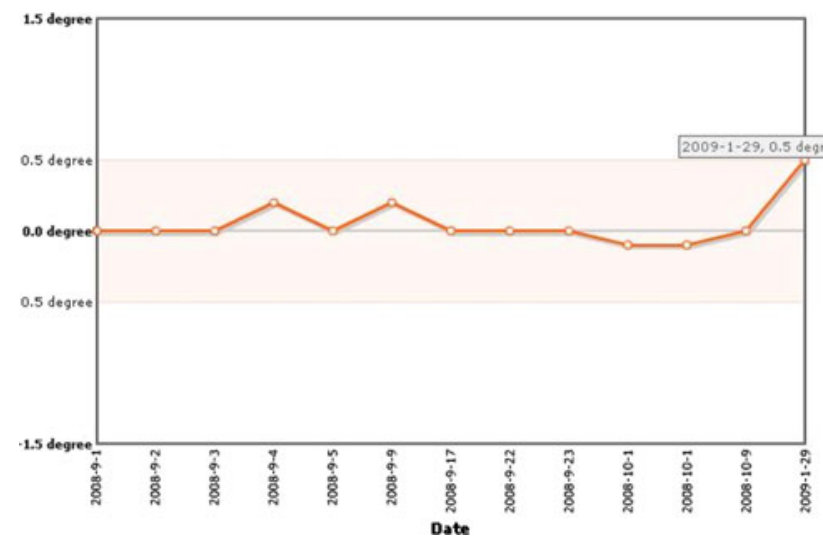

The implementation of a web based QA system at NCCC has resulted in a robust and easily managed QA program. The use of a database and trend analysis tools provides the user with the ability to draw conclusions and act on results in a more timely fashion.

Web based physics QA database is more powerful tool than paper documents and save more time, space and resource.

\section{Implementation of feedback guided voluntary breath hold gating for CBCT based stereotactic body radiotherapy}

Yong Peng ${ }^{1}$, Sastry Vedam ${ }^{1}$, Song Gao ${ }^{1}$, Jerimy Polf ${ }^{1}$, Ramaswamy Sadagopan ${ }^{1}$, Martin Bues ${ }^{1}$, Peter Balter ${ }^{1}$

${ }^{1}$ Department of Radiation Physics, University of Texas M.D. Anderson Cancer Center, USA

Purpose To implement feedback guided voluntary breathhold for CBCT based stereotactic body radiotherapy.

Methods and materials Eight patients with early stage lung tumours eligible for stereotactic body radiotherapy and with respiratory induced tumour motion of $>1 \mathrm{~cm}$ (determined from 4D CT) were selected for feedback guided breath hold implementation. Visual feedback was provided to assist patients in maintaining consistent breath hold levels. Multiple fast spiral CT scans were acquired, both within and between successive breath holds. A single breath hold CT was chosen as the reference. Gross tumour volume (GTV) was delineated to encompass the suspected tumour volume on all breath hold CT acquisitions. Standard margins for treatment planning were $0.8 \mathrm{~cm}(\mathrm{CTV})$ and $0.3 \mathrm{~cm}(\mathrm{PTV})$. Prior to each treatment delivery, a CBCT was acquired under a series of breathholds. Changes in volume, shape and size from reference were determined using 3D-3D matching tools. Shifts made based on 3D-3D matching were then verified through 2D-2D matching of acquired orthogonal MV breath hold images.

Results Overall median breath hold duration was $20 \mathrm{~s}$ ( $\max 52 \mathrm{~s}$; min $3 \mathrm{~s})$. CBCTs required 2-3 breathholds and were noted to be of superior quality to those obtained during normal respiration. Intra and inter breath hold reproducibility (RMS) of GTV centroid position at the time of repeat simulation was 1.2, 2.1 and $1.0 \mathrm{~mm}$ (AP, SI, LR). Inter fraction breath hold reproducibility of GTV position with respect to bony anatomy was $0.5,1.7$ and $0.4 \mathrm{~mm}$ (AP, SI, LRsystematic) and 1.3, 1.9 and $1.1 \mathrm{~mm}$ (AP, SI, LR-random).

Conclusions Respiratory feedback and image guided breath hold gating can improve the accuracy/precision of stereotactic body radiotherapy, especially for mobile tumours.
Physics and the paperless radiotherapy department

Rebecca Murry ${ }^{1}$, Mark Middleton ${ }^{1}$, Brendan Healy ${ }^{1}$

${ }^{1}$ Radiation Oncology Queensland

Radiotherapy departments are rapidly moving towards becoming 'paperless' but what does this mean for medical physicists? Physicists perform many tasks-routine quality assurance (QA) of equipment and planning systems, radiation safety, plan checking, patient specific measurements and more. The effective management of these tasks, particularly in a large department or across multiple centres, can require painstaking work to organise schedules, assign tasks and record data. At our facility, the Varian ARIA $^{\text {TM }}$ Patient Information System is used by radiation oncologists, therapists, nurses and administration to manage all aspects of a patients care.

A number of 'patients' (linac1, planning system etc) have been created to represent particular areas of routine physics work. All QA tasks associated with these patients have been scheduled to the nominated staff member to be performed at required intervals and appear in a 'Task Pad' in the order they are due to be done. Clinical tasks such as patient specific measurements and intensitymodulated radiotherapy QA are also tasked to Physics. Results from these clinical tasks are then stored within the patients electronic medical record along with all other information pertaining to their treatment.

We consider that use of ARIA ${ }^{\mathrm{TM}}$ to manage our day-to-day tasks has offered us improved data integrity and security. We are able to function within this paperless environment rather than outside of it. There is still room for improvement and we will continue to strive towards achieving an integrated physics tool able to handle all aspects of our role in the modern RT department.

Pre- and post- treatment MLC error analysis based on MLC and dynamic log files

\author{
Jacek Chojnowski $^{1,2}$, Romuald Gajewski ${ }^{1,2}$ \\ ${ }^{1}$ Westmead Cancer Care Centre, ${ }^{2}$ Institute of Medical Physics, \\ University of Sydney
}

Introduction Two methods have been developed for estimating dosimetric errors created by inaccuracies in MLC leaf positions for the sliding window IMRT.

Methods In the first pre-treatment method, a two dimensional map is generated with values of expected dosimetric error for a given assumption of an average leaf gap width uncertainty. Estimation of dosimetric errors is based on a correlation of dosimetric error with a gap width and a width uncertainty of this gap. The second posttreatment method uses dynamic log files generated by the MLC controller after executing the IMRT treatment plan. Those files are first converted into MLC files by in-house developed software. Then using a treatment planning system (TPS-Eclipse ${ }^{\circledR}$ ), original MLC files of an IMRT treatment plan can be substituted with converted dynamic $\log$ files and the dose delivered to the patient can be recalculated.

Results In the pre-treatment method, it was found that significant dosimetric errors occurred at sharp changes of gradients in dose profile. In the post-treatment method, a mean dose delivered to the CTV had differed on average by $-1.5 \%$. This relatively large discrepancy was further investigated. Importing MLC files from the TPS and exporting them back unchanged caused the TPS to miscalculate doses delivered to the CTV on average by $-1.6 \%$. After correction 
for this error, the post-treatment method shows average dose difference of $0.1 \%$.

Discussion and conclusion Two MLC error analysis methods show relatively quick and easy techniques of estimating dosimetric errors caused by inaccuracies of MLC leaf positions.

\section{Session 6B-Treatment planning}

\section{Composite dose distribution verification support for accuracy of dynamical Arc-IMRT treatment delivery}

\author{
Yang Wang ${ }^{1,2}$, Daniel Santos ${ }^{1,3}$, Yunfei $\mathrm{Hu}^{2}$ \\ ${ }^{1}$ Radiation Oncology Associates, ${ }^{2}$ University of Wollongong, \\ ${ }^{3}$ University of Sydney
}

Introduction In past most common IMRT plan the accuracy of dose delivery checked by ionization measurement or in-vivo dosimetry measurement, but the dose distribution commonly managed using 2D-Array checks, however a 2D array either ion-chamber or diode, the technique is limited to use for fixed field IMRT. First because the beam entry angle, the IMRT treatment plan need to be modified each field entry angle perpendicular to the array detectors. For second reason that the beam entry angle dependency made 2D array unable to provide composite dose distribution for whole IMRT treatment delivery. A 2D array provides IMRT QA checks the collimation accuracy only, a significant proportion of quality issues of IMRT treatment delivery such as beam delivery influenced by gantry angles, couch structure absorption cannot included, and 2D array is almost not be possible to be used for dynamic Arc-IMRT check. In this study a result of composite delivered dose distribution verification via Gaf-Chromic film image comparing analysis with planning exported dose map to verify the IMRT delivery quality/ accuracy via isodose curve comparison, point dose inspection and gamma value calculation.

Method and material A determined dose map for the cut layer exported from planning system in ASCII format and loaded into self-developed analysis system. The dose value for each position converted into equivalent darkness intensity and reconstructed as a film equivalent image we call it 'Virtual Film'. EBT film used to set in special designed cylindrical shape phantom to lay the film horizontally at treatment isocentre but avoid majority up to $99 \%$ of lateral beam entry to the film. The with a IMRT one fraction treatment deliver to the phantom, then film scanned and load into the analysis system with size registration process. The scanning signal was calibrated with linearity response to the IMRT fraction dose range. Lateral beam entry is also analysed, and the significances of influence to the dosimetry result has discussed.

Result and discussion The dosimetry analysed and overlay process between dose map and film imaging provides reliable comparison result adequately showing the analysis result, compare with in-vivo dosimetry, this dose map comparison shows more clear the dose distribution matching and discrepancy over the PTV region. Composite IMRT dose distribution analysed by a combined function component include point dose and profile curves inspection, isodose curve overlay comparing and Gamma Value calculation. This verification with cases included both ordinary fixed field IMRT and Varian RapidArc IMRT cases. The analysis results covered all clinical QA issues concerned such as for gantry angles and couch bar transmission influences. The verification provides a good commissioned IMRT clinical data test and the analysis reliability and accuracy can reach to $\pm 2 \mathrm{~mm}$ and $\pm 3 \%$ from Gamma value calculation.

Conclusion IMRT composite dose distribution verification leaded by comparing planning exported dose map with real treatment delivery exposed Gaf-Chromic film to provide full IMRT required comparison analysis result, the verification can be used for both fixed field and dynamic arc-IMRT. The verification technique developed by this study is very clinical use friendly, easy with the result shows good reliability and efficiency with QA time saving.

\section{Concept for adaptive therapy replanning}

\author{
Michael Jameson ${ }^{1,2}$, Lois Holloway ${ }^{1,2,3}$, Phil Vial $^{2,3}$, Peter Metcalfe $^{1}$ \\ ${ }^{1}$ Centre for Medical Radiation Physics, University of Wollongong, \\ ${ }^{2}$ Department of Radiation Oncology, Liverpool and Macarthur Cancer \\ Therapy Centres, ${ }^{3}$ Institute of Medical Physics, School of Physics, \\ University of Sydney
}

Adaptive radiotherapy is proposed to account for tumour changes in size, shape and location throughout a course of radiotherapy. This study proposes a method of determining if re-planning is necessary from comparison of contours. It correlates contour variation and radiobiology metrics as a surrogate of treatment plan outcome.

As a concept investigation this work uses pre-existing data from a contouring study. The variation from observer to observer on the same image data set was used to model the variation in tumour size/ location. Nine contours of gross tumour volume (GTV) were generated for five lung patients, a plan was generated for each contour. The contours were compared in volume, centre of volume and dimension metrics. The tumour control probability (TCP) was calculated for each treatment plan. Changes in TCP for each treatment plan were then correlated with contouring metrics.

It was found that the modelled variations had a measurable effect on TCP. Volume differences in GTV delineation ranged from 0.1 to $90.1 \mathrm{~cm}^{3}$. The average difference in GTV location was $0.43 \pm$ $0.22 \mathrm{~cm}$. The average difference in TCP was $2.14 \pm 2.13 \%$. The $R^{2}$ coefficient of regression for differences in GTV volume and TCP for patient two was 0.0018 while the $R^{2}$ for the same patient for differences in GTV location and TCP was 0.9034 .

There is still no consensus on the point at which a patient should be re-simulated and re-planned. This method provides a simple approach to making a quantifiable decision about a complex problem.

\section{Clinical significance of source size overestimation caused by the detector volume effect in a radiotherapy treatment planning system in 3DCRT and IMRT plans}

\section{Johnson Yuen ${ }^{1,2}$, Peter Metcalfe ${ }^{2}$, Nick Hardcastle ${ }^{2}$ \\ ${ }^{1}$ St George Hospital, ${ }^{2}$ University of Wollongong}

Introduction The 80-20 penumbra broadening due to ionisation chamber detector volume effect $[1,2]$ is between $1-2 \mathrm{~mm}$ for in megavoltage photon beams. The source size parameter in the Pinnacle $(8.0 \mathrm{~m})$ RTPS model increases with increasing penumbra width. While the detector size effect has been studied for 3DCRT [3], our study investigates the effect of the correctly selected source model parameter derived from zero volume detector data on IMRT and 3DCRT plans.

Methods The profile data was modelled with Pinnacle version 8.0. The sensitivity of the source size parameter in Pinnacle against the penumbra was characterised, and the optimum source size for each set of data was determined. 3DCRT plans, as well as inverse IMRT converted to forward-planned IMRT plans, were evaluated.

Results The sensitivity of the source size parameter in Pinnacle to the penumbra was dependent on field size and depth, and was found to fit well in a polynomial function. The sensitivity of the source size parameter to penumbra was higher at small field sizes, and for $1 \mathrm{~mm}$ 
of penumbra broadening the increase in optimum source size varied from 0.5 to $1.5 \mathrm{~mm}$.

Discussion and conclusions The effects of penumbra broadening due to detector volume on the RTPS model and clinically relevant plans were characterised. The potential benefits of obtaining datasets with minimal penumbral broadening are outlined in this study.

\section{References}

1. Laub WU, Wong T. The volume effect of detectors in the dosimetry of small fields used in IMRT. Med. Phys. 2003; 30:341-347.

2. Pappas E, Maris TG, Papadakis A, et al. Experimental determination of the effect of detector size on profile measurements in narrow photon beams. Medical Physics 2006; 33:3700-3710.

3. Garcia-Vicente F., M. J. Bejar, et al. (2004). 'Clinical impact of the detector size effect in 3D-CRT.' Radiotherapy and Oncology $74: 315-322$.

\section{An empirically motivated radiotherapy margin recipe}

Alan Herschtal ${ }^{1}$, Jessica Thomas ${ }^{1}$, Farshad Foroudi ${ }^{1}$, Tomas Kron ${ }^{1,2}$, Chris Fox ${ }^{1}$

\section{${ }^{1}$ Peter MacCallum Cancer Centre, ${ }^{2}$ RMIT University, Melbourne}

Conventional analytic approaches to margin calculation for conformal or intensity modulated radiation therapy make various assumptions about the distribution of the displacement of the target volume across treatment fractions of the radiotherapy regimen. Three important assumptions generally made and found not to hold empirically are as follows.

- Hyperfractionation - that for margin calculation purposes, the treatment regimen can be treated as consisting of an infinite number of infinitely small treatment fractions. Using software simulation studies we show that a typical 30 fraction treatment regimen results in treatment dosimetry significantly different from that of a hypothetical treatment regimen with a far larger number of extremely small fractions.

- Uniform patient displacement variation-that the variance of the random component of the patient inter-fraction displacement is equal for all patients (i.e. that the underlying treatment error is the same for all patients). Analysis of a dataset of displacement data for 150 prostate patients treated with prostate radiotherapy using online imaging, shows that some patients display greater variation in displacement than others on the treatment couch.

- Directional independence of patient displacement-that patient offsets in all three axial dimensions (AP, SI, and LR) are pairwise independent. Analysis of the same data as above shows that for prostate RT there is a strong correlation between patient displacement in the AP and SI directions.

This work develops a margin recipe that takes into consideration the breaches of these three key assumptions, and shows that such a recipe results in a $97 \%$ reduction in margin inaccuracy over recipes that don't.

\section{Session 6C-Biomedical technologies}

\section{High-level time synchronisation in epoch based, long-term} wireless on-body sensor networks

\section{Joshua Khoo ${ }^{1}$, Ma $\mathrm{Ke}^{1}$, Ian Brown ${ }^{2}$ \\ ${ }^{1}$ Department of Electrical and Computer Systems Engineering, Monash University, ${ }^{2}$ Monash Biomedical Centre of Engineering}

This work concerns a high level approach in time synchronisation among independent wireless (Bluetooth) sensor nodes based on the
Post Facto Time Synchronization design scheme. It can be shown that this novel high-level approach of synchronisation provides both suitable accuracy and robustness in a fully wireless, intelligent physiological monitoring system implementation.

Brief methodology In the current implementation of our intelligent physiological monitoring system, long-term monitoring capabilities are achieved with epoch transmissions that allow the MCU and radio link to sleep in between epochs while a low power RTC keeps track of time elapsed. Since the sensor node is sleeping, training of the undisciplined clock is non trivial. Our high-level approach is briefed below:

- When the sensor node is turned on, data is time stamped and stored in flash memory in reference to the local sensor's RTC.

- Once a predefined initial epoch has elapsed, the sensor attempts a data connection and SNTP is used to acquire the global UTC to discipline the local RTC and time stamps are corrected on the fly prior to transmission. A flag is then set in the sensor node.

- Other sensor nodes that have completed their epoch will attempt the same data connection. To significantly reduce the data terminal's reliance and usage, the time will be propagated from the sensor that has the flag set. After propagation, the initial sensor is placed to sleep and this role is repeated until time has converged (within a predefined threshold of accuracy) through the network.

To improve the accuracy of the sender-receiver propagation of time, two a priori consecutive time references of different packet lengths are generated to estimate the processing and transmission time delays.

Conclusion This method has been implemented and shown to be highly energy efficient in our monitoring system achieving a convergence of $\sim 80 \mathrm{~s}$ after 3 epochs whilst minimising unnecessary radio use.

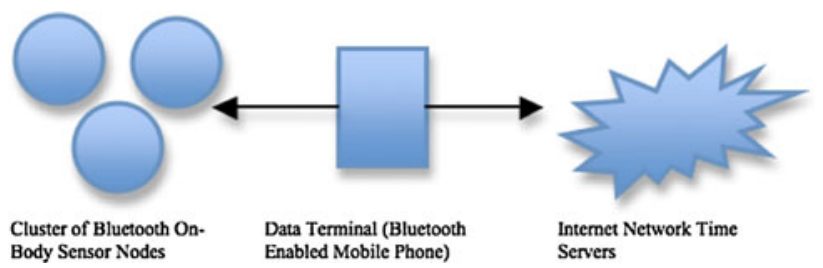

Fig. 1 Overview of the network topology

Wireless orthopaedic pin for bone healing and growth: antenna development

\section{Parisa Zakavi $^{1}$, Nemai Chandra Karmakar ${ }^{1}$, Ian Griggs ${ }^{2}$ \\ ${ }^{1}$ Monash University, Department of Electrical and Computer Systems} Engineering, ${ }^{2}$ Echidna Pins

A wireless battery operated automated orthopaedic pin to replace a manually manoeuvred bone fixation pin is proposed. The electronic pin (Fig. 1a) comprises of a body of revolution slotted waveguide antenna, a rectifying circuit, a charge storage circuit, a microcontroller and a stepper motor to elongate the pin by pushing the moveable half (Fig. 1b). The slot antenna receives microwave power from a transmitter located outside the leg. This work presents the development of the conformal antenna.

The antenna is designed to operate at approximately $21 \mathrm{GHz}$ for all pin lengths (Fig. 2a). The gain $(8.42 \mathrm{dBi})$ and radiation pattern stay relatively constant as the pin's length is varied (Fig. 2b). It was found that by placing a small Perspex plate inside the pin, the antenna becomes circularly polarised (CP) and can operate satisfactorily for any extension of the pin due to improved return loss. Consequently, the $\mathrm{CP}$ antenna can be positioned in any direction relative to the transmitting antenna without causing deterioration in the signal power. This simplifies the task of the nurse or doctor who operates the wireless battery system dramatically. 


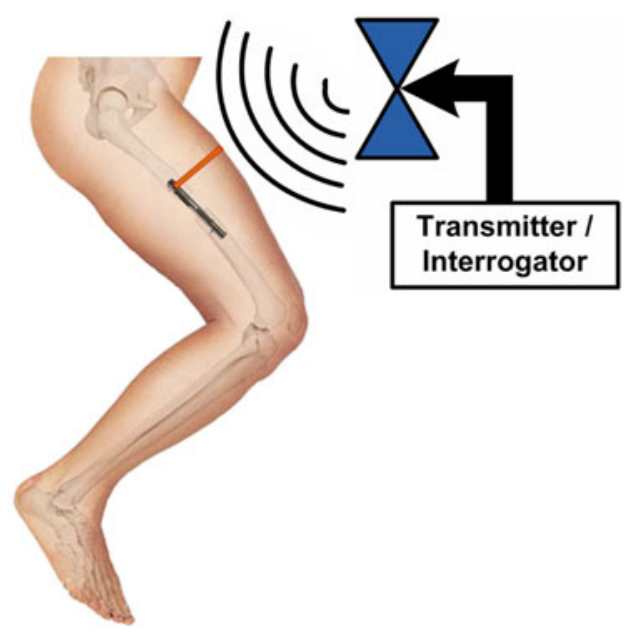

(a)

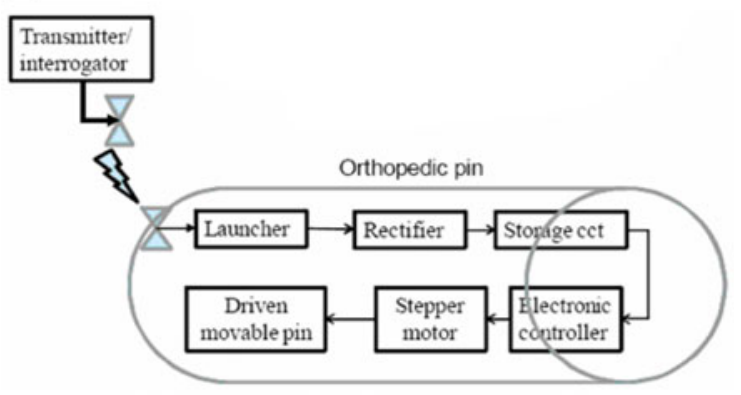

(b)

Fig. 1 a Bone fixation pin implanted in the femur. b Proposed wireless battery system
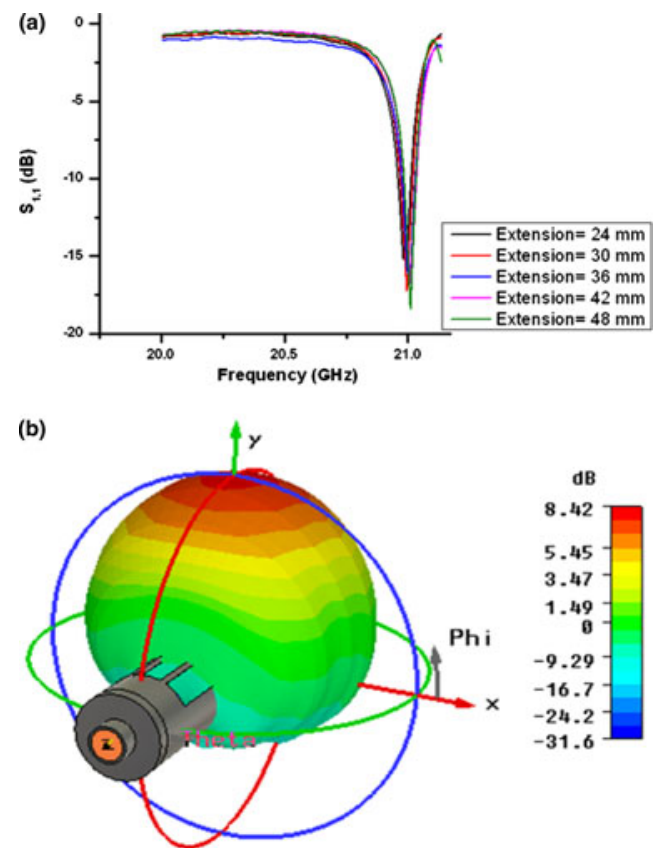

Fig. 2 a Return loss curves for selected pin lengths. b Simulated 3D far field radiation pattern
The proposed wireless pin eliminates the need for daily manipulations and operations on the human body to change the length of the pin. It makes the treatment significantly less painful for the patient and reduces the risk of infection. The full system integration is under way and will be presented at the conference.

Wireless passive radio frequency based monitoring system for sleep apnoea patient (especially paediatric patients)

Nemai Karmakar ${ }^{1}$, Yang Yang $^{1}$, Ian Brown ${ }^{1}$

${ }^{1}$ Department of Electrical and Computer System Engineering, Monash University

An innovative architecture that applies wireless technology for monitoring sleep apnoea patients is proposed. In order to overcome the limitations encountered by current sleep monitoring products, a tiny wireless medical monitoring unit is introduced to capture and convey physiological signal parameters from a patient to a base station. As is shown in Fig. 1, the proposed system consists of two building blocks: a wireless passive radio frequency transponder based on-skin monitoring probe (see Fig. 2) and a remote base station. The on-skin monitoring probe consists of a patch antenna, a voltage doubler, a signal modulator, a microcontroller and a transducer. The integrated wireless probe is light weight, low profile, hence reduces discomfort of monitoring patients. The remote base station supplies the wireless monitoring probe with enough radiated power via microwave transmitter. Working at $5.4 \mathrm{GHz}$ ISM band, microstrip patch antenna is only $5 \mathrm{~mm}$ in diameter on an antenna substrate of relative permittivity 6 . The antenna captures microwave power from the base station and converts it to $3.5 \mathrm{~V}$ DC voltage with a rectifier and a voltage doubler circuit to energise the microcontroller and the transmitter. A novel antenna model has been developed and the wireless technology is applied into the monitoring system. The realisation of the highly efficient rectenna will bring a revolution in replacing the traditional method of using on board battery. Patient can sleep at home and the physiological data can be transferred wirelessly to doctors via internet or Wi-Fi. Thus the data represent true sleeping patterns contrary to the data captured in a monitored sleeping lab. The results of the research can be widely used in other biomedical and diagnostics services both in hospitals and homes.

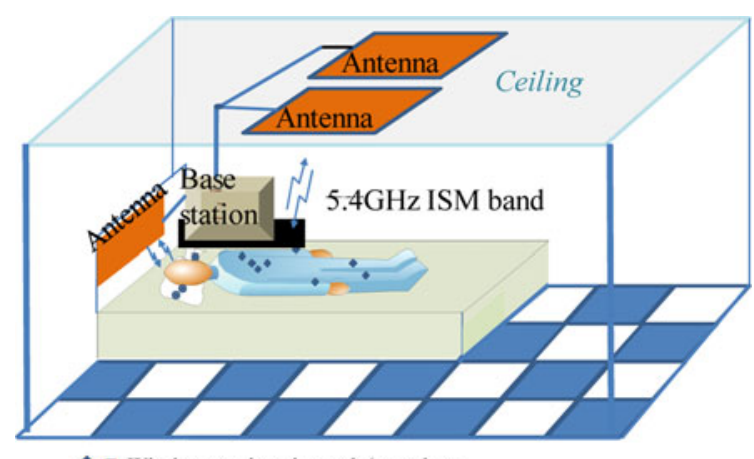

๑ Wireless passive electrode/transducer

Fig. 1 Proposed wireless RF monitoring system 


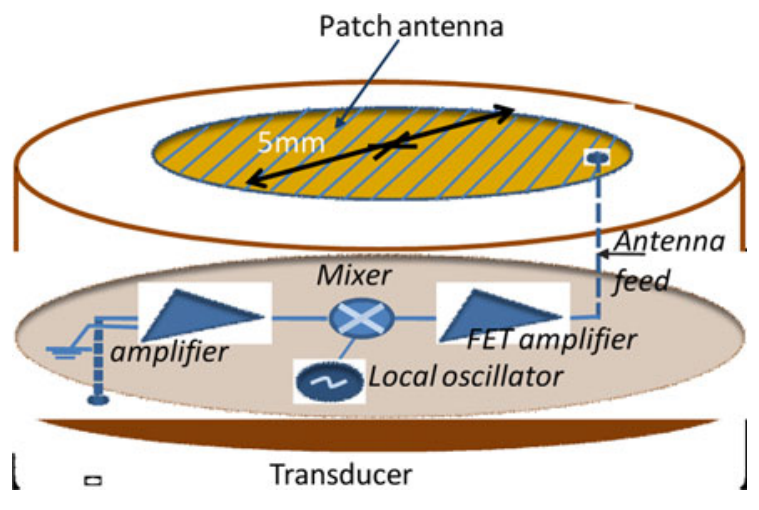

Fig. 2 Proposed wireless RF electrode/transducer

Innovate or invalidate? A review of patent filing trends in medical engineering

\author{
Alyssa Grabb ${ }^{1}$, Kathy Baker ${ }^{1}$ \\ ${ }^{1}$ Phillips Ormonde Fitzpatrick Patent and Trade Mark Attorneys
}

Patent filing data is an indicator of inventive activity. In many instances, trends in patent filing activity can be used as an indicator of investment in research and the rate of technology development. Monitoring filing trends across countries can highlight geographical regions experiencing growth and benefiting from investment in technology development, and also regions experiencing a contraction of R\&D activities. This talk will examine trends observable within patent filing data relevant to the Medical Engineering sector across a range of countries, and what this means for technology development in the sector both in Australia and abroad.

\section{Session 6D-Neonatal and paediatric medicine}

\section{Neonatal and paediatric emergency transport systems}

\section{James McCauley $^{1}$ \\ ${ }^{1}$ Biomedical Engineering, Children's Hospital at Westmead}

Since the late 1970s our department has worked with clinical staff to improve the safety and efficacy of neonatal and paediatric emergency transport by designing and producing retrieval systems for use in road vehicles and fixed or rotary wing aircraft.

Originally, these systems involved fixing gas cylinders, power supplies and various medical devices, such as transport incubators and ventilators, to the existing stretchers used in ambulances, aircraft and helicopters. Over time, these systems have evolved into three distinct sub-types being Type 1 for newborn or young infants less than $6 \mathrm{~kg}$, Type 2 for infant or young children $5-35 \mathrm{~kg}$ and Type 3 for older children $>30 \mathrm{~kg}$.

In parallel, the development of fixation, loading and ground handling systems has progressed to the point whereby the different systems are now interoperable between every road vehicle and aircraft we use. If needed, the systems can all be manually carried for deployment in remote and locations.

While the systems are designed to utilise various external power and gas sources, they can operate in stand alone mode alone for extended periods when required. Our newer power supplies are microprocessor controlled and can load shed between various internal components to extend battery life.

Such systems need to be ergonomically astute, clinically functional and incredibly reliable, as they must function in extreme conditions over long periods. At the same time they aim to meet aviation, road transport and therapeutic goods requirements. These separate regulatory frameworks often change, presenting an ongoing challenge.

\section{Paediatric versus adult technology-a BME perspective}

\section{Adrian Richards ${ }^{1}$ \\ ${ }^{1}$ Biomedical Engineering, The Women's and Children's Hospital}

The physiology and anatomy of neo-natal and paediatric patients can be very different to that of the adult population. This has a marked impact on the practice of medicine, giving rise to specialist facilities to deliver care. From the perspective of a biomedical or clinical engineer the range of specific issues faced in one of these paediatric institutions is broad and, at times, challenging. In general categories these include:

- The choices of technology are often quite limited. The larger medical technology market is specialised enough, the paediatric segment is a very small niche within it. Quite often technologies suitable for adult use simply do not transfer across.

- The range of conditions with which paediatric patients present is very different. There is a larger proportion of congenital problems that alters the technology mix substantially from an equivalent acuity adult environment.

- The care of children is highly emotive and often sees broad exposure in the public arena, for better or worse. Executive are highly media-aware and funding mechanisms rely very heavily on the charitable sector. This in itself presents its own set of technology management challenges.

- Paediatric diagnostic facilities see relatively low throughput, often just a fraction of an adult equivalent. The necessity to use sedation and anaesthesia for what may be a relatively quick and simple test on an adult is common.

- Record keeping requirements are demanding, with terms from 25 years to indefinite being prescribed. This is neither simple nor straightforward.

These issues will be discussed in some detail, with examples of how they manifest themselves and the impact that they may have on the practice of clinical engineering within the facility.

\section{Lunch session}

Optimisation of workflow/process via asset and patient tracking

\section{David Venning ${ }^{1}$}

\section{${ }^{1}$ GE Healthcare}

Along with the increase in wireless technology options for RFID in general, there are increasing use cases for applying this technology to solve problems in continually changing environments, streamline processes and automate some aspects of workflow.

The focus on RFID and in particular real time locating systems (RTLS) in health care is gaining momentum. There are many forms of RFID hardware technologies that enable RTLS systems such as: Wi-Fi, Zigbee, Passive RF, UHF, Ultra Wideband, Infrared, Ultrasound, RuBee and the list goes on. 
A quick review of basic components and terminology for RFID hardware and RTLS will be discussed.

What are the desirable characteristics of RFID hardware for the health care environment? What lessons from the past decade have been learnt in applying these technologies to health care in particular?

How do we use this technology to take on some of the day to day workflow burden of staff and at the same time create a safer work environment, improving the quality of health care to our patients.

Do we understand the current workflow practices and processes involved in delivering patient care when we look at applying this technology?

Let's have a look at some common use cases for RTLS in various hospital departments and across the hospital enterprise.

Once we have a system in place, what can be done to make better use of this system and achieve a greater return on our investment.

\section{Session 7-Plenary session}

\section{Evolving trends in academic and clinical education of medical physicists}

\section{Ervin B Podgorsak ${ }^{1}$ \\ ${ }^{1}$ McGill University, Canada}

During the past decade medical physics has become a full-fledged discipline recognised as a specialty of physics at the level of established traditional physics specialities. With the ever-increasing sophistication of equipment and techniques used in medicine, especially in imaging and radiotherapy, the need for medical physics services is growing rapidly making great demands on education of young professionals entering the field of medical physics. Accreditation of academic and clinical medical physics educational programs and certification of medical physicists are becoming important components of contemporary medical physics practice. In response to more stringent requirements for entering the medical physics profession, academic and clinical educational programs are evolving continuously to provide young professionals with academic and clinical knowledge as well as skills that will enable them to pass the rigorous certification examination and allow them to work competently as professionals in a clinical environment.

There are three basic requirements for successful academic and clinical teaching of medical physics: (1) Student must have a solid background in undergraduate physics and mathematics; (2) Teacher must be a skilled medical physicist and must know what to teach; and (3) Teacher must practice effective teaching. There are no shortcuts to education and the three requirements for medical physics teaching seem clear. Yet, fulfilling them is not a trivial task. Potential students must understand that the term 'medical physicist' implies a professional who works in a clinical environment but is a physicist first and foremost, and potential teachers must understand that effective teaching requires much knowledge of the subject, clinical experience as well as enormous dedication, skill and effort.

\section{Quality and safety in health care}

\section{Chris Baggoley ${ }^{1}$ \\ ${ }^{1}$ Australian Commission on Safety and Quality in Health Care}

There is a paradox in health care. There is no doubt that health care is improving across a large number of fronts and that Australia is a world leader in many areas. Cancer survival rates, recovery from major trauma, response to heart attacks, joint replacement therapies are just a handful of examples where clear advances have been made in recent decades.

Yet there is also evidence that patients can be harmed in the process. They can develop hospital associated infections, experience medication errors, suffer injury as the result of a fall in hospital, can have their clinical deterioration go unrecognised. They can receive care that varies from that which is recommended by best available evidence.

This presentation will consider this paradox and will detail the actions of the Australian Commission on Safety and Quality in Health Care as it responds to the challenge of achieving a vision of safe and high quality care for Australia.

\section{Session 9-Plenary session}

\section{ECRI Institute's assessments of health care technology}

\section{James P Keller, $\mathbf{J r}^{1}$}

${ }^{1}$ Health Technology Evaluation and Safety, ECRI Institute

For over 40 years, ECRI Institute has been dedicated to bringing the discipline of applied scientific research to discover which medical procedures, devices, drugs, and processes are best, all in the interest of helping health care organisations improve patient care. Much of that work has revolved around ECRI Institute's laboratory- and evidence-based assessments of health care technology. The laboratory-based assessments are published as comparative evaluations in the highly respected Health Devices journal. ECRI Institute's evidence-based technology assessments are published on the Web site for its Healthcare Technology Assessment Information Service (HTAIS) and in customised reports for health care providers, insurance companies, and government agencies like the US Centers for Medicare and Medicaid Services.

This presentation will review the processes and methodologies used to conduct ECRI Institute's health care technology comparative evaluations and evidence-based assessments. This will include a discussion of typical findings from ECRI Institute's research, commentary on the challenges associated with this work, and a review of where its technology assessment services will likely be heading in the future. Sample topics will include infusion pumps, imaging systems, computer-based medical technology, intravascular stents, and radiation therapy. The presentation will wrap up with a discussion of how ECRI Institute's technology assessment experience can be applied in the typical health care institution's technology planning, procurement, and forecasting initiatives.

\section{Health technology and horizon scanning}

\section{Brian Richards ${ }^{1}$ \\ ${ }^{1}$ Health Technology and Medical Services Group, Department of Health and Ageing}

This presentation provides an overview of horizon scanning, within the international and Australian context, and where it fits into the broader health technology assessment process. The presentation outlines the approach to horizon scanning taken by the Health Policy Advisory Committee on Technology (HealthPACT) in Australia and briefly looks at the implication of new technologies (including radiation oncology equipment) on workforce as well future expansion in treatment capacity. 


\section{Session 10A-Regional medical physics}

\section{Appropriate technology for developing countries}

\section{Barry Allen ${ }^{1}$}

${ }^{1}$ University of New South Wales

The Health Technology and Training Task Group (HTTTG) was set up by the International Union of Physics and Engineers in Medicine (IUPESM) at the 2006 world congress in Seoul, with the objective of supporting the development of appropriate technology in rural communities in developing countries. Our recent investigation in 2007 into the health services in the Mekong Delta of Vietnam led to the development of recommendations that call for action on several fronts. Action Groups (AG) were set up within the HTTTG to address each recommendation. These Action Groups have the task of reviewing commercially available equipment, evaluating whether such equipment is appropriate for the designated task, and if not, developing specifications and proposals new R\&D that would satisfy the specifications.

AGs have been formed on the following topics:

- Telemedicine;

- X-ray source and imaging;

- ultrasound source and imaging;

- basic blood chemistry analysis;

- palliative radiotherapy;

- cervical screening.

HTTTG will use these reports as independent, expert opinion on which to develop further actions in the way forward and to provide the basis for funding requests. These reports are available at www.iupesm.org.

The first phase of HTTTG action was to identify key technical needs in a specific location. This has now been completed in part with reference to the Mekong Delta region of Vietnam, Philippines and Vanuatu. The second phase is now to recommend appropriate equipment and training to satisfy these identified needs. The third phase is to support the purchase or development of the required equipment and its installation as a demonstration model in a selected location, e.g. the Mekong Delta. The widespread implementation of the technology then becomes a matter for the relevant Ministry of Health and international funding organisations.

The first workshop on Palliative Radiotherapy for Cancer in Saigon, 2008 was a major step in the second phase of the HTTTG program. The results of this workshop will be reviewed and recommendations presented.

\section{Asia-Pacific special interest group discussion}

\section{May Whitaker ${ }^{1}$ \\ ${ }^{1}$ SSWAHS}

Abstract not available at time of printing.

\section{Session 10B-Medical physics}

Study of the peripheral dose in paediatric head and neck patients using a low cost, easily modifiable phantom, the 'jelly-baby'

Paul White ${ }^{1}$, Stephen Bosi ${ }^{1}$, Meg Schneider ${ }^{1}$, Robert Smee ${ }^{1}$

${ }^{1}$ Department of Radiation Oncology, Prince of Wales Hospital
Introduction Peripheral dose to organs-at-risk in paediatric head and neck patients has been estimated using models and large-sized phantoms [1]. Mansur et al. [2], using stacks of water-equivalent slabs approximating the dimensions of a 3-year old, showed that peripheral dose was too phantom geometry dependent to allow extrapolation of paediatric dose from adult work. Given this geometry dependence, this work aims to compare a water-equivalent stack to a more anthropomorphic paediatric phantom.

Method Two phantoms were produced, scaled to approximate the size of a 3-4 year old, fitted with TLDs and a CC04 ion chamber at locations approximating thyroid, breast, ovaries and testes.

- 'Block-Baby': Stacks of water-equivalent slabs (c.f. ref [2]).

- 'Jelly-baby': Stack of flexible bolus cut to conform to coronal CT contours of a 3.9 year old. Water equivalence of the flexible bolus was measured.

Both were subjected to realistic treatment plans and peripheral doses measured.

Results and conclusions Preliminary results (below) indicate that phantom peripheral doses (relative to isocentre dose) were consistent within $\sim 10-20 \%$. The discrepancy between Jelly-Baby CC04 and TLD testes doses is a rough guide to the uncertainty $(<\sim 20 \%)$.

\begin{tabular}{lll}
\hline Organ & Block-Baby CC04 & Jelly-Baby TLD \\
\hline Thyroid & $1.73 \%$ & $1.51 \%$ \\
Breast & $0.52 \%$ & $1.44 \%$ \\
Ovaries & $0.16 \%$ & $0.20 \%$ \\
Testes & $0.03 \%$ & $0.11 \%$ \\
Testes & & $0.13 \%(\mathrm{CC} 04)$ \\
\hline
\end{tabular}

The paper will include subsequent measurements, analysis and other treatments (traditional conformal, stereotatic etc.). Jelly-Baby can be modified to include heterogeneities (e.g. cavities and lung).

\section{References}

1. Klein E.E., et al. 2006. Med. Phys. 33 2525-2531.

2. Mansur D.B. et al. 2007. Radiother. Oncol. 82 179-184.

\section{Cross-sectional human tissue equivalent phantoms}

Tony De Souza-Daw ${ }^{1}$, Paul Junor ${ }^{1}$, Robert Stewart ${ }^{2}$, Philip M Lewis ${ }^{3}$, Richard Manasseh ${ }^{2}$, Jerome Maller ${ }^{4}$

${ }^{1}$ Department of Electronic Engineering, La Trobe University, ${ }^{2}$ Fluid Dynamics Group, CSIRO Materials Science and Engineering Division, ${ }^{3}$ Department of Neurosurgery, Alfred Hospital, ${ }^{4}$ Brain Stimulation and Neuroimaging, Alfred Psychiatry Research Centre

There is a need for on-site, research laboratory production of crosssectional ultrasound phantoms such as transcranial phantoms. TissueEquivalent phantoms (also called tissue-mimicking phantoms) are generally difficult to construct and materials can be difficult to source. A procedure for an easy-to-construct phantom is presented in a formation that can represent the cross-section of an ultrasound examination with numerous tissue layers.

In the procedure, each layer consists of agar or gelatin as the basic congelation substance, with graphite or calcium (bones) added as the attenuator. The concentrations of the ingredients in each layer differ for each tissue. These layers have been constructed such that the entire ultrasound interrogation mimics what would be observed in 
practice. For example, the transcranial cross-sectional phantom includes the following layers: scalp, bone, cerebrospinal fluid and brain tissue.

In real scenarios, regions of interested must be analysed through bodily tissue. A layered phantom provides a close approximation to what is seen in practice. The properties of the layers can be varied to see what affect they have on the region under study. Visual and signal processing characterisations of layers can be performed to establish relative acoustic properties.

Phantoms constructed using the procedures developed are amenable to mimic almost any tissue cross-section encountered in a human examination. This will prove valuable for researchers wanting to undertake in vitro studies under credible conditions.

\section{Near-catheter dosimetry of a HDR brachytherapy source using Gafchromic ${ }^{\circledR}$ film}

\author{
David Odgers $^{1,2}$, Mamoon Haque $e^{1,2}$ \\ ${ }^{1}$ Department of Radiation Oncology, Royal Prince Alfred Hospital, \\ ${ }^{2}$ Institute of Medical Physics, School of Physics, University of \\ Sydney
}

Introduction An endobronchial irradiation is a specific form of intraluminal irradiation by high dose rate (HDR) brachytherapy and can be delivered using as little as one afterloading catheter. The common dose prescriptions for this form of treatment ranges from 8 to $10 \mathrm{~Gy}$ at a distance of $10 \mathrm{~mm}$. A difficulty that arises from these dose prescriptions is the production of extreme high dose gradients, especially near the active catheter involving tissues, making evaluation of these dose distributions difficult. The aim of this experiment was to evaluate the efficacy of Gafchromic film to measure the doses given by a single-catheter brachytherapy treatment at the surface of the catheter.

Methods Measurements were performed using Gafchromic EBT2, MD-55 and HD-810 films (ISP, Wayne, New Jersey, USA). A Perspex jig was designed to hold a catheter in a small groove adjacent to a wider groove to hold a piece of Gafchromic film against the catheter surface. Calibration of the films were performed by using Virtual Water (Civco Medical Solutions, Kalona, Iowa, USA) sheets to place strips of Gafchromic film at the $10 \mathrm{~mm}$ dose prescription distance. Analysis of the Gafchromic film strips was performed by scanning them using an Epson Expression 10000 XL flatbed scanner and RIT113 v5.2 software to investigate the dose profiles.

Results The measurements at the catheter surface showed that for dose prescriptions at $10 \mathrm{~mm}$, and source step sizes of $2.5,5$ and $10 \mathrm{~mm}$, hot spots of up to $37,37.5$ and 43 times the prescription dose could be observed, respectively.

Discussion and conclusions The hot spots produced by an endobronchial treatment simulation were successfully measured using Gafchromic film, showing that the magnitude of the hot spots increases with step size. The next step is to analyse the measured dose profiles to the catheter surface and compare with the treatment planning system. Further analysis will also be carried out comparing the hot spots produced by multi-catheter treatments (such as prostate treatments) with this form of single catheter treatment.

\section{Three-dimensional dosimetry imaging technique of I-125 plaque for eye cancer treatment}

M Weaver ${ }^{1}$, M Petasecca ${ }^{1}$, MLF Lerch ${ }^{1}$, D Cutajar ${ }^{1}$, D Franklin $^{1}$, $\mathrm{J}_{\text {Green }}{ }^{1}$, MG Carolan ${ }^{1,3}$, P Metcalf ${ }^{1}$, M Conway ${ }^{4}$, J Jakubek ${ }^{2}$, $S_{\text {Pospisil }}^{2}$, T Kron ${ }^{5}$, AB Rosenfeld ${ }^{1}$
${ }^{1}$ Centre for Medical Radiation Physics, University of Wollongong,

${ }^{2}$ Institute of Experimental and Applied Physics, Czech Technical University in Prague, Czech Republic, ${ }^{3}$ Illawarra Cancer Care Centre, ${ }^{4}$ Sydney Eye Hospital, Faculty of Medicine, The University of Sydney, ${ }^{5}$ Peter MacCallum Cancer Centre

Introduction There are approximately five in 1,000,000 cases of uveal melanomas per year, ${ }^{[1]}$ making melanoma and squamous cell carcinoma the most prevalent ocular malignancies in adults. Disease morbidity and cost to the community is significant with almost $50 \%$ of patients losing vision or the eye due to the disease and/or the treatment. ${ }^{[2,3]}$ Brachytherapy, using radioactive eye plaques, is the preferred method of treatment for patients with such ocular malignancies. However, where tumours are small and/or close to critical structures such as the optical nerve, customised plaques and robust quality assurance in dosimetry is required.

Material and method A new approach to I-125 eye plaque brachytherapy $3 \mathrm{D}$ dosimetry was developed, based on a pixelated silicon detector. High spatial resolution dosimetric results are achieved in real time by capturing a number of $2 \mathrm{D}$ dose images with the MEDIPIX 2 pixelated detector using a PMMA eye phantom and PixelMan software, and subsequently generating a 3D reconstruction of the dose date.

Results Eye plaques loaded with five or ten seeds were investigated and the MEDIPIX 2 detector was calibrated relative to the TG-43 protocol. The 2D dose images with $50 \mu \mathrm{m}$ spatial resolution were obtained at a variety of plaque-sensor distances and angles within the eye phantom, with the plaque loaded with either five or ten seeds. The reconstructed 3D images were in close agreement with a dose simulator for this plaque developed at CMRP.
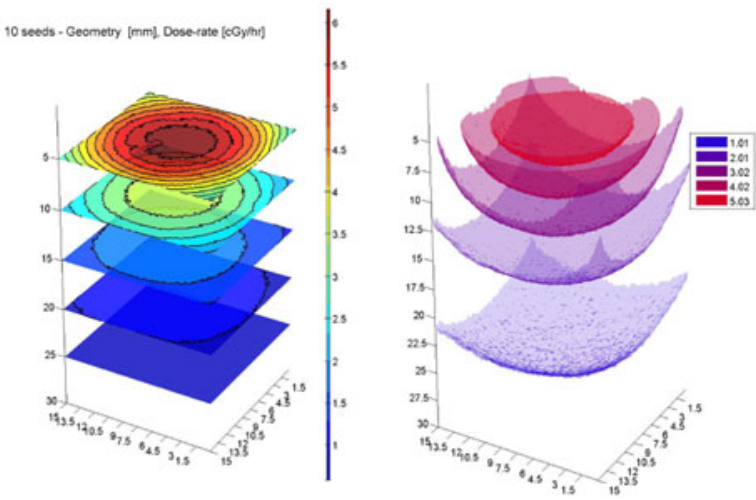

\section{D Isodose surfaces in terms of counts and dose-rate for plaque loaded with $10 \mathrm{I}-125$ seeds}

This unique system under development at CMRP is designed to offer eye plaque brachytherapy clinical quality assurance checks for optimised treatment and will allow the development of new medical procedures for the realisation of patient-specific plaques based on the $3 \mathrm{D}$ reconstruction of the dose effectively delivered in the eye.

\section{References}

1. Singh AD, Kivelä T. (2005) 'The collaborative ocular melanoma study’, Ophthalmol Clin North Am, 18(1), 129-42, 2005.

2. Damato B 'Treatment of primary intraocular melanoma', Expert Rev Anticancer Ther., 6(4), 493-506, 2006.

3. Granero, D., et al., 'Dosimetric studies of the $15 \mathrm{~mm}$ ROPES eye plaque', Med. Phys., 31, 3330-3336, 2004. 


\section{Session 10C-Device performance on the market}

\author{
Quality and safety-hazards and recalls
}

\author{
James P Keller, $\mathbf{J r}^{1}$ \\ ${ }^{1}$ Health Technology Evaluation and Safety, ECRI Institute
}

The management of medical device hazards and recalls has become an increasingly challenging responsibility for hospitals. The number of hazard and recall reports that hospitals need to process has grown significantly over the last number of years. ECRI Institute's alerting program, Health Devices Alerts, published 241 medical device alerts in 2001. We expect to publish well over 2,000 alerts in 2009. Fortunately many alerts are straightforward, involving product labelling changes or revised user instructions. However, there are a growing number of serious alerts that if not acted on quickly can have a significant negative impact on patient safety. Examples of recently published alerts from ECRI Institute include (1) serious injuries to infants from mismatched heated humidifiers and heated-wire breathing circuits, (2) failure to defibrillate and critical delays in therapy from incorrect insertion of defibrillator cables, and (3) reprocessing problems causing patient infection risks with flexible endoscopes.

This presentation will discuss ECRI Institute's perspectives on the best practices for managing medical device hazards and recalls. It will cover use of standard device nomenclature to help identify products affected by alerts in a hospital's inventory, assignment of roles and responsibilities for addressing problems reported in hazard and recall documents, and will provide recommendations for improving the timeliness and accountability related to medical device hazards and recalls. The presentation will also include a review of ECRI Institute's list of top ten medical technology hazards and recalls.

Usability and ergonomics-why and how we use them in the investigation of medical device adverse event reports

\author{
Ying Huang ${ }^{1}$, Jorge Enrique Garcia ${ }^{2}$ \\ ${ }^{1}$ Biomaterials and Engineering, Office of Laboratories and Scientific \\ Services, Therapeutic Goods Administration
}

The concepts of usability and ergonomics (also called human factors engineering) have been well established for more than half a century. There are many Australian and international standards regarding the usability and ergonomics used in aviation, consumer electronics, road vehicles and computer software industries. While the importance of usability and ergonomics is generally understood, the first standard applying these aspects in the design of medical devices was only first introduced in 1993.

The 'Essential Principles' (in the Australian Medical Device Regulations) demand that the measurement, monitoring and display scale of the device must be designed and produced in accordance with ergonomic principles, having regard to the intended purpose of the device. However, research has shown that lack of usability of medical device is a strongly contributing cause of use errors ${ }^{1}$. Moreover, the TGA continues to receive incident and adverse event reports in which the cause is attributed to 'user error' when these errors may have been prevented, or made less severe through use of design measures.

In this presentation, several case studies of investigations of incidents reported to the TGA's Medical Device Incident Report Investigation Scheme (IRIS) will be presented and analysed. The use of usability standards as guidance during the investigations will be discussed. Finally, it will be argued that although user error contributed to the cause of the incidents, a more thorough use of usability and ergonomic principles during the product development process could have either prevented or reduced the severity of the outcomes.

\section{Reference}

1. AAMI/FDA Conference 1995, Human Factors in Medical Devices: Design, Regulation, and Patient Safety. http://www.fda. gov/MedicalDevices/DeviceRegulationandGuidance/Postmarket Requirements/HumanFactors/ucm126018.htm.

Post market medical devices regulation-case studies on post market medical devices incident investigation and monitoring process

\section{Senthilvel Natarajan ${ }^{1}$}

${ }^{1}$ Market Vigilance Monitoring Section, Office of Devices, Blood and Tissues, Therapeutic Goods Administration

A series of case studies are presented to demonstrate and highlight the Australian post market medical devices regulatory system.

It is important that medical devices remain of good quality, as safe as possible and perform as intended throughout their life. The post market vigilance and monitoring program is part of the overall regulation system that aims to ensure this occurs.

Post market Medical Devices Incident Reporting and Investigating Scheme (IRIS) Case studies will be used to demonstrate how medical device incident reports are investigated by the TGA. The presentation will provide details on how the outcome is determined by the TGA through examination of the incident, information obtained from the reporter, sponsor/manufacturer and other reliable sources and testing in the TGA's laboratories.

Post market monitoring approach (targeted review) Case studies will also be used to demonstrate how the TGA monitors safety, quality and performance of devices on the market through targeted reviews (e.g. monitoring safety and performance of ophthalmic and dermatology lasers, wrist blood pressure monitors, blood glucometers, etc). These case studies will show how product reviews are initiated, what evidence is required and gathered to ensure that medical devices continue to be of good quality, safe and perform as intended.

Sustainable improvements in incident management-it can be done

Carmel Brennan, Liza Dale ${ }^{1}$

${ }^{1}$ ACT Health

An innovative approach to the implementation of an incident management system across the complete spectrum of a Health Care Service has achieved bold outcomes.

The approach used to implement this project was a deviation from previous implementation practices within the organisation. A deliberate decision was made to establish it as a change management project which focused on people and processes rather than technology. A robust project planning methodology was rigorously applied to all stages of the project. The unique skill mix in the project team of an Information Technology (IT) Project Manager /Registered Nurse and a Process Engineer ensured that issues and challenges were addressed in an innovative manner.

This project achieved national recognition for its change management approach. It well exceeded previous records in achieving training attendance. By project completion over $80 \%$ (3000) of all staff had attended face to face training. This had a direct impact on the acceptance of the new incident reporting methodology. A significant cultural shift in how incidents are recognised and reported has been identified through the improved quality of the reports received. 
Incident reports have increased by $150 \%$ since the introduction of the system.

Focusing the project on the people and process rather than the technology allowed a culture of change management to permeate the project, ensuring that staff were never forgotten. This approach has resulted in a sustained improvement in incident reporting and management. The unique skills of an Engineer aligned with a clinician IT Project Manager ensured that thorough business analysis was undertaken so the project could be assured of addressing the actual problems the clinicians faced. The use of an industry standard project management methodology combined with the support of a mentor was vital to achieving bold outcomes.

\section{Session 10D—Maintenance engineering}

Reliability centred maintenance: a maintenance strategy for biomedical engineering

\section{Rebecca Jucha ${ }^{1}$, Phillip Thorburn ${ }^{1}$}

${ }^{1}$ Biomedical Engineering Department, Royal Adelaide Hospital

Introduction Is AS 3551 effective? Is 'run to failure' an effective and valid alternative?

Over the past 3 years the Biomedical Engineering Department (BME) at the Royal Adelaide Hospital (RAH) has been exploring the advantages of using the principles and methodologies of reliabilitycentred maintenance (RCM) to improve the management of the hospital's medical equipment assets.

Discussion and conclusions RCM II is a logical, robust process which identifies appropriate risk based maintenance strategies for a device or system. RCM is also a good aid to identifying design deficiencies in a piece of equipment and in the policies and procedures governing its operation and maintenance.

The RCM II methodology will be applied to a simple circuit to demonstrate its use in developing effective maintenance strategies and to draw conclusions on how the application of RCM can improve biomedical engineering practice.

The cost associated with implementing RCM makes it prohibitive for most hospital biomedical engineering departments working in isolation. However, the airline industry shows that there is an affordable means of implementing RCM in biomedical engineering.

\section{AS 3551 Testing of mains cables—affect of cable length} on results

\section{PL O’Meley ${ }^{1}$, E Dela Cruz ${ }^{1}$}

${ }^{1}$ Sydney South West Area Health Service, Biomedical Engineering

Background AS 3551 requires measurement of Earth Resistance, Earth Leakage Current and on occasions Insulation Resistance. Earth leakage current represents the potential hazard current during failure of the earth conductor and is the sum of two components-leakage from the device (potential hazard) and the mains cable itself (largely harmless). The investigation aimed to decouple these components to determine the value of the true hazard.

Method We measured earth resistance, earth leakage and insulation resistance for a large number of mains cables of varying lengths on a small number of devices. We used both new cables and old cables to try and determine any degradation of performance with age. As earth resistance varied with each measurement, we took several measurements on each cable.
Results

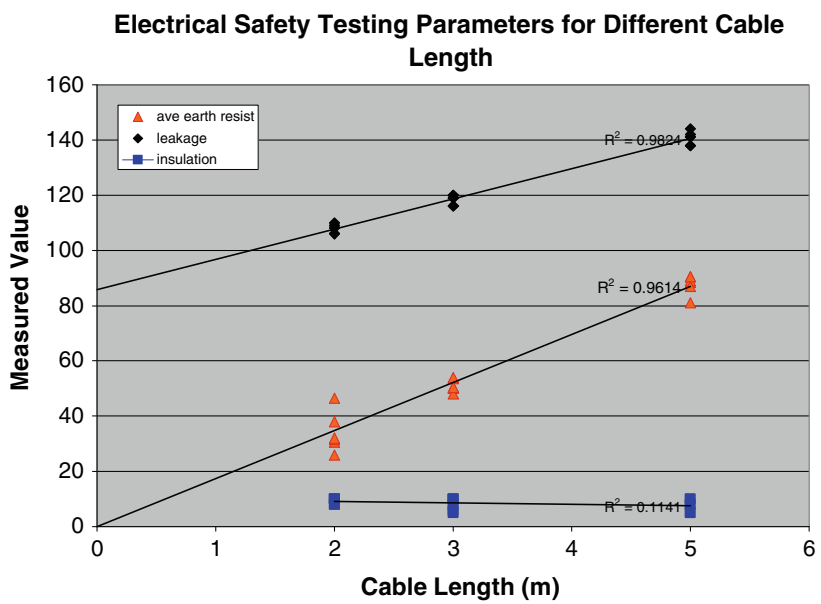

Fig. 1 Plot of electrical safety parameters versus mains cable length. Plot shows device contribution to Leakage Current is 85 micro-amps (estimated zero cable length value from line of best fit). With cable length zero, Earth Resistance is zero (to be expected). Insulation Resistance is largely invariant with mains cable length

The results show that the actual potential hazard current (Earth Leakage Current) is lower than measured with a cable connected. The device hazard current can be predicted from two measurements at different cable lengths. Insulation Resistance is unaffected by cable length. Earth Resistance varies with cable length-as expected.

Conclusion Potential hazard current (earth leakage) is dependent on device-and measurement of which is obscured by the non-hazardous contribution of the cable. Understanding this can assist in making better determinations of required actions following high measurements.

Adjusting planned inspection intervals using risk management principles

\section{Ken Wilson ${ }^{1}$, Gordon Brown ${ }^{1}$}

${ }^{1}$ Queensland Health

The risk associated with use of medical equipment that is not fully functional is determined by assessing the consequence and likelihood of a possible failure mode. Consequence is the easier of these elements to assess. Likelihood is very dependent of the scope that the risk management process covers.

Using data obtained from previous maintenance experience, it is possible to calculate the mean time between failures of the different failure modes and the level of confidence a user could expect to have that the equipment is fully functional when they are about to use it. This paper will discuss in more detail the process followed, will detail the level of acceptance from clinical colleagues, and some likely outcomes for different device types of medical equipment.

\section{Lunch session}

\section{Does DICOM compliance guarantee interoperability?}

\section{Andrew Barnhill ${ }^{1}$}

${ }^{1}$ GE Healthcare

Picture this case study.

Current imaging department consists of CT/X-Ray/Ultrasound and PACS. 
Customer is going to add an MRI and a small printer.

Head of Department has asked you to connect everything up and get it all working. Would also like to know what back up plan we have in case of the PACS going off line. So what should you do prior to the equipment arriving. Is it all plug and play or are there any surprises? All vendors have said that their particular equipment is DICOM compliant, and have mentioned that they have DICOM conformance statements to prove it.

As you leave the office the Head of Dept says 'I want to implement Structured reports, could you make sure it all works when the new equipment arrives. I spoke to the sales guys and they said that the equipment is DICOM compliant.' 'You don't have a problem with that?'

Over the next thirty minutes I will go through what needs to be done in regards to DICOM conformance comparison, what you need to look at in the conformance statements, do you need to talk to the IT department, do you need to spend any more money to get DICOM working and do you need to talk to the vendors about anything.

\section{Session 11A-TEAP workshop}

\section{Medical physics teaching, accreditation, and certification in North America}

\section{Ervin B Podgorsak ${ }^{1}$}

${ }^{1}$ McGill University, Canada

The United States and Canada have well developed clinical and academic medical physics services, strong national medical physics organisations, excellent research productivity, and well-respected certification process for medical physicists. The cooperation and interaction between American and Canadian medical physicists are excellent despite the significant differences in health care financing between the two countries. Most Canadian medical physicists, in addition to supporting the Canadian Organization of Medical Physicists (COMP) that counts over 500 members, are also active members of the American Association of Physicists in Medicine (AAPM) which has over 6000 members. The AAPM is thus not only an American national organisation for medical physics; it also plays a role of a regional medical physics organisation.

During the past 20 years the Commission on Accreditation of Medical Physics Educational Programs (CAMPEP) has been accrediting academic and clinical educational programs in medical physics. Accreditation or re-accreditation of a program, typically given for 5 years, implies strict adherence to AAPM Task Group Report 79 for academic programs and AAPM Task Group Report 90 for clinical residency programs. Currently 22 academic and 35 residency programs are accredited by the CAMPEP.

In the future graduation from an accredited program will become a requirement for admission to writing the professional certification examination in medical physics. Candidates meeting appropriate academic and clinical credentials can write the certification examination in radiation oncology physics, diagnostic radiology physics, or nuclear medicine physics. In the United States the examination is given by the American Board of Radiology (ABR), in Canada by the Canadian College of Physicists in Medicine.

\section{TEAP radiation oncology}

\section{Steve Howlett ${ }^{1}$}

${ }^{1}$ ACPSEM

Abstract not available at time of printing.

\section{TEAP nuclear medicine}

\author{
Stefan Eberl ${ }^{1}$ \\ ${ }^{1}$ Royal Prince Alfred Hospital \\ Abstract not available at time of printing.
}

TEAP radiology

\section{John Cormack ${ }^{1}$}

${ }^{1}$ ACPSEM

Abstract not available at time of printing.

Canada and Australia—mutual recognition?

\author{
Lisa Duggan ${ }^{1}$ \\ ${ }^{1}$ Calvary Mater Newcastle \\ Abstract not available at time of printing.
}

\section{Session 11B-Medical physics}

O KiloVolate: where art thou? The dosimetric investigation of LMA electron cones for the treatment of superficial cancers

Michael Grace ${ }^{1}$, Stewart Griffin ${ }^{1}$, Alicia Puran ${ }^{1}$, Erin Redfearn ${ }^{1}$, Daniel Santos ${ }^{1}$, Richard Short ${ }^{1}$, Guangli Song ${ }^{1}$, Yang Wang ${ }^{1}$

${ }^{1}$ Radiation Oncology Associates

For the treatment of superficial or mildly invasive cancers, the choice has largely been between low energy X-rays, from 50 to $300 \mathrm{kV}$, on an orthovoltage or superficial machine, or via high energy electrons on a linac. The use of the Valencia skin applicator ${ }^{1}$ using a HDR brachytherapy source adds another option.

In the absence of a superficial/orthovoltage machine, small superficial cancers (that is, less than $3 \mathrm{~cm}$ in diameter) are treated with high energy electrons, with initial beam shaping at the electron applicator, and customised lead shielding on the skin ${ }^{2}$. Lateral electronic disequilibrium changes the characteristics of the electron beam for small field sizes shifting the R100 value closer to the surface, and increasing the beam's penumbra. This technique involves the manufacture of customised lead and wax shielding, and frequently requires in vivo dosimetry for verification. Additionally, setup times are involved, lengthy and with some patient discomfort.

The use of LMA cones for electron therapy is a novel alternative. The cones, varying from 1.5 to $3.0 \mathrm{~cm}$ in diameter, are seated in the standard electron applicator, with a source-to-cone distance of $98 \mathrm{~cm}$. No additional shielding is applied on the patient's skin, and the cone is constructed based on the individual shape of the lesion. Measurements via film and in water confirm that the beam properties are similar to previous treatments techniques. Although not a clinical procedure at present, this technique will be introduced as it is simple to construct, plan and treat, and saves time in all aspects of the process.

\section{References}

1. Device produced by Nucletron

2. MD Perez, et al, 'Dosimetry of small electron fields shaped by lead', Australasian Physical \& Engineering Sciences in Medicine Volume 26 Number 3, 2003, 119-124 
Radiation mis-information and informed consent of participants in human research

\section{Martin Caon ${ }^{1}$ \\ ${ }^{1}$ School of Nursing and Midwifery, Flinders University}

Clinical trials involving humans often include medical imaging. Participants are provided with a 'participant information statement' (PIS) as part of their informed consent. The information is first scrutinised by a Human Research Ethics Committee.

It is evident that many PIS are written by people without expertise in radiation. This assertion is made on the basis of the poor standard of the information about radiation that is usually included. Given that sponsoring Drug Companies continue to present PI statements with a low standard of radiation information, one can presume that HRECs without expertise in radiation, fail to identify these deficiencies.

Many participants are also patients and their medical imaging is seen as routine, and accepted as normal standard of care. Nevertheless, providing erroneous radiation information does not assist the process of informed consent. Some examples of information taken from PIS ${ }^{1}$ that are: designed to allay fear and anxiety; wrong; imprecise; and unhelpful will be discussed.

Multinational drug companies design trial protocols 'for a living'. One could expect their PIS to be 'state of the art'. Yet some radiation statements are so obviously wrong that (in my opinion) they have not been seen by a physicist. The information about radiation in the PIS should be reviewed by an expert to ensure that it is sound.

\section{Reference}

1. Caon, M. 2008, Radiation information and informed consent for clinical trials, J. Radiol. Prot. 28, 415-422.

\section{Fifteen years since the development of the Australian gamma surgical probe for applications in lymphoscintigraphy and lymphobiopsy}

\section{Carl Muñoz-Ferrada ${ }^{1,2}$}

${ }^{1}$ Gammasonics Institute for Medical Research,

${ }^{2}$ University of Wollongong

The aim of this work is to $\mathrm{R} \& \mathrm{D}$ a precise gamma probe to perform lymphoscintigraphy and lymphobiopsy in surgery.

When I took to researching into the techniques and the instrument described, I was not aware of all the hidden complexities. (a) It is not just a gamma probe application. It is the combination of the design of the gamma probe, chose of the correct detector and crystal, (b) understanding of the colloids and the procedures, (c) the suitable patients for lymphobiopsy procedures, (d) the most suitable activity and volume for the injection, (e) the injection time, (f) the time of the actual surgery and (g) the benefit to the patients. Furthermore, we have to understand the radiological implications of exposure to surgeons and auxiliary staff during the surgical procedure.

We have produced 700 gamma probes and all of them are in operation in almost every corner of the world. The number of patients benefiting from the procedure bypasses a million.

The gamma probe today is used for breast cancer, melanoma, gynaecology, colon and rectum and other surgical applications. In reality, the limitations are in the hands of the surgeon and the nuclear medicine department.

\section{References}

Muñoz-Ferrada, C.A. 'Lymphosintography Study'. Paper 2005.

Muñoz-Ferrada, C. 'Lymphoscintography and lymphobiopsy for Breast Cancer and Melonoma: Abstract of Nine Years of Research' 2007.

Gillet, D.J., 'Sentinel Node Excision: Technique and Results', presented in Australasian College of Surgeons Conference 1999.

Gillett DJ, Dias $M$ and Molland G: Sentinel Node excision: Techniques and getting results, ANZ J Surg 69: A10 1999

Gillett DJ, Janu NC and Molland G: Sentinel node biopsy in breast cancer, Submitted for publication, July 1999.

Morton DL, Wen DR, Wong JH, et al. Technical details of intraoperative lymphatic mapping for early stage melanoma. Arch Surg 1992; 127:392-399.

Morton DL, Wen D-R, Wong JH, et al: Technical details of intraoperative lymphatic mapping for early stage melanoma. Arch Surg 1992:127:392-399.

Uren, R.F. 'Lymphatic Drainage of the skin and breast', Harwood Academic Publishers 1999. 90-5702-410-1.

\section{Dosimetric evaluation of CBCT images for adaptive IMRT/IGRT treatment planning}

\section{Chuan-Dong Wen ${ }^{1}$ \\ ${ }^{1}$ WP Holman Clinic, Royal Hobart Hospital}

Introduction Since the implementation of cone beam CT (CBCT) to verify treatment position for cancer patients undergoing IGRT/IMRT in our centre, the $3 \mathrm{D}$ CBCT data become readily available for an adaptive planning and treatment process. CBCT have advantages over conventional $\mathrm{CT}$ on reflecting patient internal organ temporal movement and inter-fractional treatment volume changes during tight-margined IMRT treatment course. This study presents our preliminary dosimetric evaluation of clinical CBCT images for adaptive planning with Pinnacle ${ }^{3}$ - Philips Medical Systems treatment planning system (TPS) at Royal Hobart Hospital.

Methods A series phantoms studies have been carried out using similar setup parameters on both CBCT and conventional CT systems to verify the characteristics of CT numbers of different materials and different scan modes, to examine the uniformity in axial plane and linearity along scan axis, and to establish the correlation of CT\# to density in the CBCT image dataset. Patient-specific CBCT data was imported into Pinnacle ${ }^{3}$ TPS and co-registered with the original planning CT data, before dose computation was carried out using CBCT data. Dose distributions in both phantom and patient were analysed and compared on both CBCT and CT datasets using Pinnacle $^{3}$ version $8.0 \mathrm{~m}$. CBCT data used in this study was acquired by Varian Medical Systems CBCT version 2.1.5.2.

Results Scans on uniformity phantoms indicated that CT\# uniformity and linearity on axial slices were acceptable in two scan modes capable of head and neck, and pelvis, fitted with full fan (FF) and half fan (HF) filters, except a crescent-shaped artefact with CT\# variation of more than $40 \mathrm{HU}$ emerged when scan length was beyond a threshold value. Artefacts displayed on both phantom and clinical images due to complex internal scatter within the cane beam and were depended on scan volume. Dose distributions in homogenous phantom suggested CBCT data can be used for dose calculation when scan length was relatively short and scatter from medium outside cone were adequate. CT\# to tissue density correlation was established through inter-comparison of CT/CBCT scans of ScanPlas ${ }^{\circledR}$ phantom and this relationship was scan volume dependent. 
Discussion and conclusions $\mathrm{CBCT}$ dosimetric characteristics are more complex in comparing with of those of CT due to scattering effect within its scan volume. Various artefacts exist on some scan modes but can be minimised by optimising scanning parameters. CBCT data can be used with caution for clinical plan verification and validation in an adaptive IMRT process provided proper CT\# to density table has been established and the scan parameters have been correctly applied.

\section{References}

1. A quality assurance program for the on-board imager ${ }^{\circledR}$ Sua Yoo et al Med. Phys 33 (11), Nov 2006.

2. On-Board Imager (OBI) Reference Guide-Varian Medical Systems Ver 1.3 March 2007.

3. Catphan ${ }^{\circledR} 500$ and 600 Manual Copyright ${ }^{\odot} 2006$ the Phantom Laboratory.

4. Establishment of Relationship between CT Number and relative electron density, R A Chappell, internal report to W P Holman Clinic, Royal Hobart Hospital, March 31, 1995.

\section{Session 11C-Biomedical engineering education and partnerships}

\section{The Melbourne School of Engineering and the Department of Clinical Engineering teaching partnership}

Emmanuel Koumoundouros ${ }^{1}$, W Jack Davie ${ }^{2}$, Michael J Fowler ${ }^{2}$

${ }^{1}$ Melbourne School of Engineering, The University of Melbourne, ${ }^{2}$ Department of Clinical Engineering, Royal Melbourne Hospital

The Melbourne School of Engineering (MSE), University of Melbourne has established new Biomedical Engineering (BE) undergraduate and postgraduate programs. The MSE has developed a partnership with the Department of Clinical Engineering (CE), Royal Melbourne Hospital $(\mathrm{RMH})$ to provide lectures, workshops, student projects and a professional work experience program to the BE programs.

The CE department has had a unique and direct input in this new program by developing specialised lectures and workshops to the BE capstone subject. The specialised lectures include the role of Therapeutic Goods Administration and the role of the Australian Standards in the regulation of Medical Devices. The workshops are based on electrical safety testing that specifically covers the electrical standards. Students undertake safety testing workshops in CE and then visit clinical departments where they gain an understanding of the practical aspects of implementing the standards in clinical areas.

While it is not uncommon for Biomedical Engineering Departments to provide general lectures on Biomedical Engineering, student projects and vacation work, RMH CE allocates student projects based on the standards, typically on the application of the standards to the testing and verification of equipment performance. $\mathrm{CE}$ also offers vacation work to senior students as part of the students' professional development.

This partnership is beneficial to students and the Biomedical Engineers with the course providing an inceptive for the Engineers to understand and question the standards, improve their communication skills and enhance their leadership potential.

\section{The Medical Device Partnering Program}

\section{Karen J Reynolds ${ }^{1}$}

${ }^{1}$ Flinders Medical Devices and Technologies, Flinders University
The Medical Device Partnering Program (MDPP), established in South Australia to provide a streamlined approach to the medical device product development process from early stage concepts right through to manufactured products, has been running since July 2008. The Medical Device Partnering Program is end-user and market driven and engages researchers, clinicians, client services and companies. The program provides a platform for identifying and assessing new product opportunities with clinical relevance, undertakes earlystage technical exploration projects, coordinates and manages relationships between stakeholders, and provides advice and assistance along the product development pipeline.

In the first 12 months since the Program was launched, over 40 companies and inventors have approached the MDPP for involvement in the Program. This presentation will provide an insight into the program models, and an update on current activities.

\section{Biomedical training survey: who wants what?}

Anne-Louise Smith ${ }^{1,2}$, Karen J Reynolds ${ }^{1}$

${ }^{1}$ Flinders Medical Devices and Technologies, Flinders University, ${ }^{2}$ Flinders Biomedical Engineering, Flinders Medical Centre

Flinders University and Flinders Medical Centre Biomedical Engineering are involved in different levels of training for biomedical engineering technical staff. The university provides tertiary biomedical engineering courses for those requiring professional engineering education, whereas the FMC department is more involved at the hands-on technical level. Opportunities exist for extension of the currently available training, but these should be driven by industry needs. An online survey was conducted to assess the further training needs of hospital-based biomedical engineers and technical staff.

Responses from 99 people in the biomedical field were collected over 1 year. Respondents were mainly from Australia (28\%), India (27\%) and Middle East (20\%) and their previous education was generally at Bachelor level (47\%). All except one wanted more training. The main reasons given were to work better $(75 \%)$ and for career and promotion (64\%). Other questions in the survey covered the areas and level of training required, and suitable course structures.

The results of this survey have been used during a redevelopment of the Flinders University course offerings in biomedical engineering, and also to shape an online course for providing further practical technical training for people working in the industry.

Student competency guide for biomedical engineers (clinical engineering stream)

\section{Steve Somerwil \\ ${ }^{1}$ Mercy Health}

The Victorian Biomedical Engineers (clinical engineering stream) requested a guide for measuring biomedical engineering student's competency after they completed University or TAFE.

The suggested competency document includes the scope of the guide, who should complete it and the competencies expected of the candidate. Further it outlines the responsibility of all involved parties including the management of the organisation conducting the 16 week competency program. The guide also gives the expected resources that are required to conduct the competency exercise and briefly gives the desired outcomes from the program. The guide indicates several examples of documents expected to be completed 
during the competency program. The final section gives an indication on how to measure the competency of the student.

The document suggests that a certificate be issued to the student upon successful completion of this guide, which could be nationally recognised for all employers, private or public, and also by Engineers Australia.

\section{Session 11D—AS 3551 Workshop}

\section{Mains power cable maintenance strategies}

Graham Elsegood $^{1}$, Phillip Thorburn ${ }^{1}$

${ }^{1}$ Biomedical Engineering Department, Royal Adelaide Hospital

Introduction Medical device mains power cables can present a major hazard for patients, staff, operators and visitors in the hospital and homecare environment. According to Australian and New Zealand standards AS/NZS 3551 [1] the testing of medical devices with associated power cable is recommended with a 'tested' identification tag attached at prescribed time intervals (usually 12 months). This predetermined testing interval does not prevent the device power cable from failing at some later time, even the same day, due to the impact of its 'operating environment'. To improve this situation an alternative replacement cable strategy has been investigated, developed and assessed.

Methods Assessment of power cable in-service use and worst case measurement data was collated and analysed.

Results Electrical parameter measurements and Failure Mode and Effects Analysis (FMEA) revealed an alarming failure of the 'test and tag' protocol.

Discussion Analysis of data collected suggests that the current controls are ineffective in reducing the risk to patients and staff.

Conclusions The 'testing and tagging' regime for mains power cables does not prevent damage or operational failure and may give device users a false sense of security. Consequently resources are being wasted on 'testing and tagging'. Alternative strategies need to be instigated that effectively manage the risk of functional failures of cables that have a safety consequence.

\section{Reference}

1. Standards Australia/Standards New Zealand, AS/NZS 3551:2004 Technical management programs for medical devices.

\section{Risk management of medical equipment using AS 4360}

\section{PL O’Meley ${ }^{1}, \mathrm{~V}$ Chin $^{2}$, K Belle ${ }^{2}$}

${ }^{1}$ Sydney South West Area Health Service, Biomedical Engineering, ${ }^{2}$ ACT Health

Background Historically, risk ratings of medical devices have used 'scoring systems' which are arbitrary, subjective and self-serving. We need a risk rating system that is simple, objective, repeatable and testable and able to be applied to a wide range of devices and circumstances. AS 4360:2004 is based on likelihood, outcome and frequency but the challenge is, finding the requisite data and having a consistent way to evaluate it.

Method We developed a generic device model with 6 elements: operator, utilities, device, patient, service, and environment. This is a fault-tree analysis of failure events. More detail can be incorporated as required with sub-categories (utilities can have power, water, gas and network included). Risk mitigation can then be employed where needed.

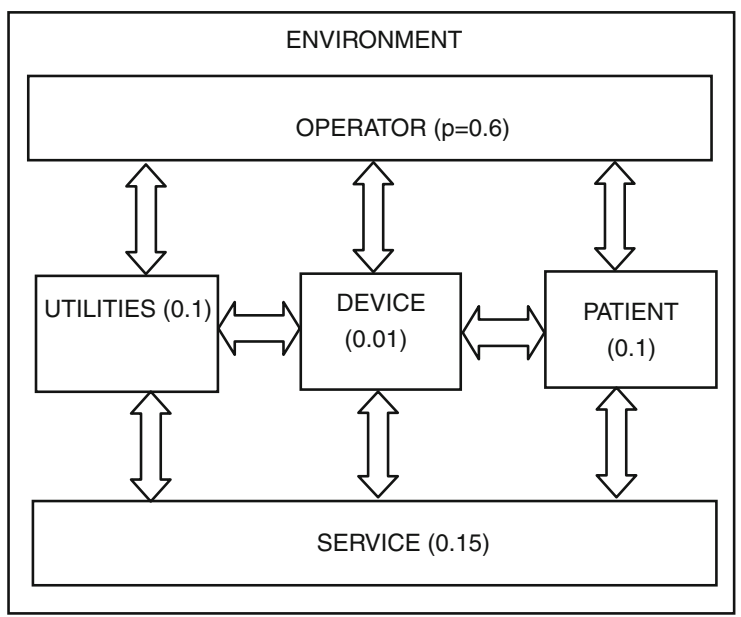

Fig. 1 Generic device failure type model (top of fault tree) - showing failure type classifications and relative contributions to death outcome for an ICU ventilator. Total probability is less than 1.0 due to 'unknown' category failure events

Table 1 Event failure risk matrix - showing likelihood of adverse outcomes on ICU Ventilator during monthly emergency generator test

\begin{tabular}{|c|c|c|c|c|}
\hline \multirow{3}{*}{$\begin{array}{l}\text { Frequency_monthly } \\
\text { Likelihood }\end{array}$} & \multicolumn{4}{|c|}{$\begin{array}{l}\text { EVENT RISK MATRIX-power } \\
\text { failure }\end{array}$} \\
\hline & \multicolumn{4}{|c|}{ Consequence } \\
\hline & Death & Major & Minor & Trivial \\
\hline Near certain $(P>0.9)$ & & & & $\mathrm{X}$ \\
\hline Common $(P=0.01)$ & & & $\mathrm{X}$ & \\
\hline Uncommon $(P=0.0001)$ & & $\mathrm{X}$ & & \\
\hline Impossible $(P=0.000001)$ & $X$ & & & \\
\hline
\end{tabular}

Multiple matrices are required for complete risk assessment using each failure type

In analysing risk we determine the likelihood of death, major injury, minor injury, or no injury for each failure category. We then determine a frequency of the failure event to determine the absolute risk for a given failure event. A spreadsheet tool was developed to aid analysis and to characterise overall risk for a given device in all failure modes.

Results We have identified: (1) Generic device failure event classes, (2) Suggested generic likelihood and frequency descriptions. Using this method we can: (a) Show how to recognise a truly dangerous event from Risk Matrix plot, and (b) Show how to identify a high risk device (and classify other devices) from a Risk Rating Histogram based on relative probabilities of failure in device categories.

Conclusion Methods outlined in AS 4360 can be combined with standard fault-tree analysis to determine risk for medical devices. Using standard descriptions and classifications can aid in data collection from this device characterised by histogram.

Risk management of medical equipment using AS 4360—an empirical approach

\section{PL O’Meley ${ }^{1}, \mathrm{~K}$ Belle $^{2}$, V Chin ${ }^{2}$}

${ }^{1}$ Sydney South West Area Health Service, Biomedical Engineering, ${ }^{2}$ ACT Health 
Background In the companion paper we describe a new Risk Management method for medical devices using AS 4360 and FTA coupled with a standard device model. The missing element is the raw data but local databases have too few incidents or insufficient detail. Method We used FDA MAUDE database to review medical device incidents. We used the number Death events per device type to obtain the likelihood (L) of death consequence (CD) for each device. We then estimated worldwide device population by extrapolation thus giving the frequency $(\mathrm{F})$ of device events on a per device basis. The combined probability $(\mathrm{pCD}=\mathrm{L} \times \mathrm{F}$ ) is then used to locate the actual risk in the Risk Assessment Matrix (Table 1). Risks of lesser consequences were estimated to rise with decreasing severity. Note that MAUDE has declining data reliability with declining incident severity. Further, we classified each MAUDE report into its Failure Category Type to construct the histogram (Fig. 1). We note considerable difficulty in determining failure category based on data in MAUDE.

Table 1 Risk assessment matrix showing relative risk for defibrillators and patient lifter

\begin{tabular}{|c|c|c|c|c|}
\hline \multirow{2}{*}{$\begin{array}{l}\text { OVERAL RISK } \\
\text { MATRIX } \\
\text { Likelihood (L) }\end{array}$} & \multicolumn{4}{|l|}{ Consequence $(\mathrm{C})$} \\
\hline & Death $(\mathrm{CD})$ & $\begin{array}{l}\text { Major } \\
(\mathrm{CM})\end{array}$ & $\begin{array}{l}\text { Minor } \\
(\mathrm{Cm})\end{array}$ & $\begin{array}{l}\text { Trivial } \\
(\mathrm{CT})\end{array}$ \\
\hline $\begin{array}{l}\text { Near certain } \\
\quad(P>0.1)\end{array}$ & & & Defib* & $\begin{array}{l}\text { Defib*, } \\
\text { Lifter* }\end{array}$ \\
\hline $\begin{array}{l}\text { Common } \\
\qquad(P>0.01)\end{array}$ & & Defib* & Lifter* & \\
\hline $\begin{array}{l}\text { Uncommon } \\
\qquad(P>0.0001)\end{array}$ & $\begin{array}{l}\text { Defib } \\
\qquad(P=0.000129)\end{array}$ & Lifter* & & \\
\hline $\begin{array}{l}\text { Very uncommon } \\
\qquad(P>0.000001)\end{array}$ & $\begin{array}{l}\text { Lifter } \\
\qquad(P=0.000003)\end{array}$ & & & \\
\hline
\end{tabular}

Risk of death is as determined from MAUDE data. Risk of lesser consequences is estimated $(*)$ and rises with decreasing severity in the normal fashion

Off the scale (ignore where $P<0.000001$ )

Results For defibs, there were 3000 events and 45 deaths. For lifters, 148 events and 2.1 deaths. Estimated worldwide population of defib is 350,000 and lifters is 700,000. From this data we constructed Table 1 and Fig. 1.

\section{Relative Frequency of Death associated with Failure Category for Defib and Lifter}

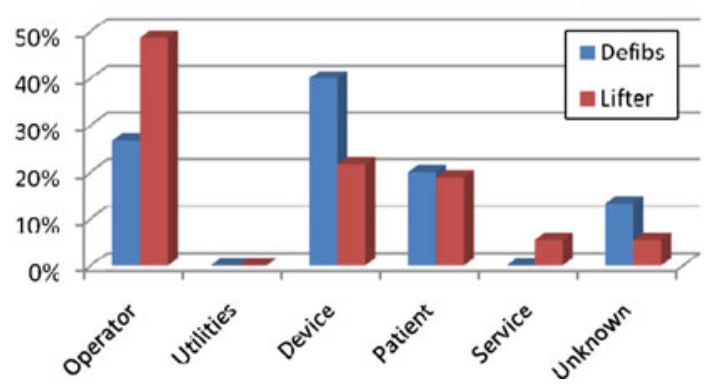

Fig. 1 Relative frequency of device failure categories for Death outcome on defibrillators and patient lifters. Showing death from device failure is more common in defibs than with patient lifter. Highest columns indicate greatest need for risk mitigation efforts

Without adequate reporting on lesser consequences all devices can seem like high risk devices as death dominates the reported events.
Conclusion We found MAUDE is a useful starting point for quantitative risk analysis. Quantified risk assessments allow prioritisation of effort and resources and histogram shows how the effort should be directed. Thus there is a need to gather information on failure categories and all adverse outcomes in equipment databases.

\section{Performance and safety inspections}

\section{Clifford Stephenson ${ }^{1}$}

${ }^{1}$ Northern Sydney Central Coast Health

Why do we routinely check medical equipment, is it effective and how do we know?

Is the risk managed by performance verification of benefit to the organisation, and how does it relate to the other risks the organisation faces?

We are all expected to routinely check medical equipment for both performance and electrical safety every 12 months, some items are checked more frequently and others not at all. Some use various risk systems to adjust the periods between checks while most rely on AS 3551's stated maximum period if a risk management system is not used, but how many refer to the manufacturer's specifications for deciding the period.

There are many risks facing an organisation, of which clinical engineering is only one small facet. Extra resources are difficult to obtain, and quite often can be cut for little reason other than to help the organisation meet budget. This is while the organisation continually expects greater outputs. Under these conditions, performance verification, and to some extent, preventative maintenance are usually the casualties. The priority becomes repairs as this has a more immediate impact on bed availability.

We need to be able to quantify the risks the organisation has and present to management in a way that allows the organisation to make valid assessments of all risks in order to decide where scarce resources should be allocated. This is very difficult when most large organisations have difficulty in developing a good overall list of its risks and their relative ratings.

\section{Session 12A-Training and education TEAP workshop}

Ervin Podgorsak ${ }^{1}$, Steve Howlett ${ }^{2}$, Stefan Eberl ${ }^{3}$, Ray Budd ${ }^{4}$, John Turner ${ }^{5}$, Lisa Duggan ${ }^{6}$, Anne Perkins ${ }^{2}$, Brian Thomas ${ }^{\text {, }}$ Mark West ${ }^{8}$

${ }^{1}$ McGill University, ${ }^{2}$ ACPSEM, ${ }^{3}$ Royal Prince Alfred Hospital, ${ }^{4}$ Safeway Radiation Safety Consultants, ${ }^{5}$ Medical Physics Services, ${ }^{6}$ Calvary Mater Newcastle, ${ }^{7}$ Queensland University of Technology, ${ }^{8}$ Queensland Health

Abstract not available at time of printing.

\section{Session 12B-Radiotherapy dosimetry}

Gafchromic EBT2 film—the new generation film for radiotherapy dosimetry
Martin J Butson ${ }^{1,2,3}$, Tsang Cheung ${ }^{1}$, Peter KN Yu ${ }^{1}$
${ }^{1}$ Department of Physics and Materials Science, City University of Hong Kong, ${ }^{2}$ Department of Medical Physics, Illawarra Cancer Care Centre, ${ }^{3}$ Centre for Medical Radiation Physics, University of Wollongong 
Gafchromic EBT2, radiochromic film is the newest X-ray dosimetry film from ISP corporation and has undergone design changes from its predecessor, EBT1. These changes have been aimed improving a few aspects of its dosimetric ability. The main differences include the incorporation of a new yellow dye into the active layer and a new style of substrate and over laminate material The yellow dye is incorporated for two reasons: (1) to improve the UV exposure sensitivity and (2) to provide a more accurate baseline for dosimetry to minimise effects from scanner variations. The new design for substrate and over laminate has also been performed to provide a more flexible but robust film design which will not be damaged as easily during cutting processes. Results have shown that the new films absorption spectra looks significantly different to the older EBT1 film due to both the use of the new yellow dye as well as different construction process. However, by looking at net optical density changes due to X-ray radiation the results show a match to the existing EBT film showing the utilisation of the same sensitive chemicals for dosimetry. Other dosimetric properties have been investigated and will be reported for Gafchromic EBT2, Radiochromic film.

\section{Matching Varian beams on an Elekta accelerator for primary standard dosimetry: comparing modelled spectra and measured PDDs}

\section{Jessica Lye ${ }^{1}$ \\ ${ }^{1}$ Australian Radiation Protection and Nuclear Safety Agency}

ARPANSA has a new Medical Standards Linac (an Elekta Synergy Platform) at ARPANSA. The purpose is to establish a direct megavoltage calibration service for radiotherapy dosimetry, instead of relying on calibrations against Co-60. The new linac has multiple $6 \mathrm{MV}$ beams, so one has been matched in energy to a Varian high energy platform accelerator. The $\mathrm{D}_{20,10}$ values are matched to $0.5 \%$. Full $\mathrm{MC}$ models of the Varian accelerator and the matched Elekta accelerator accurately predict the measured PDDs and profiles, but show significantly different spectra at the water surface. This is mainly attributed to the difference in target thickness in the two accelerators.

\section{Results of the 2009 Dosimetry Survey of Australian Radiotherapy Centres}

\section{Rhonda Brown, Duncan Butler ${ }^{1}$ \\ ${ }^{1}$ Australian Radiation Protection and Nuclear Safety Agency}

In 2009 ARPANSA conducted a survey of Australian radiotherapy centres. The aim of the survey was threefold: (1) To identify megavoltage photon and electron beam qualities in clinical use, (2) to obtain information on kilovoltage beam qualities and dosimetry protocols in clinical use, and (3) identify emerging areas where dosimetric standards may be required (such as high dose rate brachytherapy). Responses were received from more than $70 \%$ of radiotherapy centres. Results will be presented indicating beam qualities ( $\mathrm{kV}$ and $\mathrm{MV}$ ), dosimetry protocols, ion chambers and treatment modalities in clinical use.

\section{The feasibility of an air core scintillation dosimeter array: experiment and simulation}

Pourandokht Naseri ${ }^{1}$, Jamil Lambert ${ }^{1}$, David Robert McKenzie ${ }^{1}$, Natalka Suchowerska ${ }^{1,2}$

${ }^{1}$ School of Physics, University of Sydney, ${ }^{2}$ Department of Radiation Oncology, Royal Prince Alfred Hospital
The performance of a new type of scintillation dosimeter for use in external beam radiotherapy is examined by experimental measurements and Monte Carlo (MC) simulation. A $6 \mathrm{MV}$ photon radiotherapeutic beam is used in both experiment and simulation. The dosimeter has a small sensitive volume with a silvered air core light guide and is designed for use in arrays and in high dose gradients. The air core light guide avoids the generation of the Cerenkov background that would be produced in a conventional optical fibre. The MC EGSnrc program was used to determine the impact of the silver and the air on the dose deposited in the sensitive volume. It was found that for thicknesses of less than $1 \mu \mathrm{m}$, the dose deposited in the scintillator was not dependent on the silver thickness. The presence of air in the light guide had no effect. MC was also used to determine the effect of a nearby dosimeter of the same type on the dose deposited. To within the precision of the simulation, no such proximity effects were detectable for silver thicknesses less than $1 \mu \mathrm{m}$. Even for silver thickness of $100 \mu \mathrm{m}$ the largest proximity effect was less than $5 \%$. Experimental studies of the proximity effects in both longitudinal and lateral orientations with respect to the beam confirmed that no proximity effects could be detected to within experimental error of less than $1 \%$. These results confirm the suitability of this dosimeter design for use in close packed arrays in external beam dosimetry.

\section{Session 12C-Workforce planning}

\section{The future of health care-a more integrated workforce?}

\section{Liza Dale ${ }^{1}$}

${ }^{1}$ ACT Health

Patient safety has come along way from the days of pointing the finger at the clinician at the 'active' or sharp end of a health care error. James Reason and others have placed the responsibility for 'human error' squarely on the shoulders of latent (root) causes that can be prevented only by adjustments to systems and processes. The health care workforce is struggling to make the changes required due to critical staff skill gaps and an increasing health care demand. Where can we turn to find the appropriate skills to assist in this important task?

Engineers working in the health care environment have their own challenges-utilised only when required and ill understood by the health professionals they assist. They are looking for ways to enhance themselves professionally, but finding little opportunity.

In June 2005 the US Institute of Medicine and National Academy of Science released their latest in a series of health care process improvement studies entitled 'Building a better delivery system: A new Engineering/ Healthcare partnership'. The link has been recognised, we need to take the back to the future approach and work together.

I have been employed in the Patient Safety and Quality Unit of a major teaching hospital as part of the multidisciplinary quality team. Not only has this been personally rewarding but the systematic engineering approach of analysing issues has provided clarity and a way forward for some of the intractable complex issues that health care professionals have struggled to navigate.

The involvement of an engineer in patient safety initiatives works best in partnership with clinicians, providing a balance between a system focus without losing clinical emphasis. It takes some adaptation for both the clinicians and the engineers to realise the benefits of this integrated working approach. This should be the way of the future.

\section{Area biomedical staffing model-update}

PL O’Meley ${ }^{1}, \mathrm{~K}$ Belle ${ }^{2}$, V Chin ${ }^{2}$

${ }^{1}$ Sydney South West Area Health Service, Biomedical Engineering,

${ }^{2}$ ACT Health 
Background At EPSM-ABEC-2007 one of the authors presented a model for minimum staffing of Biomed Support Services across multiple facilities. Since then, research by other services has been conducted and the results are presented here.

Method The model uses workload to predict required staffing, including front-line supervision but excluding advanced services (e.g. Rad Onc, Renal). Recently, we collected data from ACT Health and Northern Tasmania, namely: number of devices, distances to workshop, numbers and averages of repair and test times. This data was compared to the model.

\section{Results}

Table 1 ACT Health (6FTE in 2008)

\begin{tabular}{ll}
\hline & TCH \\
\hline Beds & 575 \\
Predicted devices & 5257 \\
Actual devices & 4747 \\
Pred. repairs & 2105 \\
Actual repairs & 2495 \\
Pred. TO & 5.3 \\
Pred. STO & 1.8 \\
Pred. admin & 1.4 \\
Pred. tot FTE & 8.5 \\
\hline
\end{tabular}

The volume of work predicted is lower than the actual reported-and a gap of 1.5 FTE is predicted. The model also suggests more Admin and STO support is required. Similar results were obtained in Tasmania (more than 1.5 FTE gap exists).

Table 2 Northern Tasmania (4.0 FTE for 2006-latest complete data)

\begin{tabular}{lllll}
\hline & Burnie & Mersey & Launceston $^{\mathrm{a}}$ & Total \\
\hline Beds & 157 & 115 & 339 & 611 \\
Pred. devices & 670 & 400 & 2700 & 3770 \\
Actual devices & 337 & 237 & 1612 & 2086 \\
Pred. repairs & 268 & 160 & 1080 & 1508 \\
Actual repairs & 274 & 241 & 1022 & 1537 \\
Pred. TO & 1.2 & 0.4 & 2.2 & 3.8 \\
Pred. STO & 0.4 & 0.15 & 0.72 & 1.27 \\
Pred. admin & 0.1 & 0.1 & 0.4 & 0.8 \\
Pred. tot FTE & 1.7 & 0.7 & 3.3 & 5.7 \\
\hline
\end{tabular}

$\overline{{ }^{a} \text { Indicates workshop location from which other facilities are serviced }}$

Conclusion The results show where gaps exist in staffing at the sites reviewed. As the predicted resources are based on a minimum level of service, these gaps represent a risk to patient safety and we expect over time to see more breakdowns, longer repair times and an aging crumbling equipment fleet.

\section{A BC health checklist: integrating business continuity management in health care}

\section{Christine Miller ${ }^{1}$}

\section{${ }^{1}$ B 4 Crisis}

Business continuity (BC) arrangements help better prepare health care providers in dealing with non-routine business disruptions (crises).
Business continuity, risk, incident and emergency management frameworks and planning should be integrated to maximise organisational resilience. Data on why business continuity management $(\mathrm{BCM})$ is a good investment will be presented. Business impact analysis (BIA) is the foundation to best practice in BCM. Undertaking business impact analyses offers opportunities to review returning to 'business as usual', as well as during times where key resources such as infrastructure, information and communications technology (ICT) and people are unavailable or less available than usual. The BIA discussion includes 'mapping' both internal and external dependencies, such as supply chains and medical technology. The paper includes lessons learnt through critical infrastructure protection and examples of their application to health care and medical technology. Participants will be provided with a checklist to use when reviewing their organisation's business continuity preparedness.

\section{Generic biomedical department organisational structure}

\section{PL O’Meley ${ }^{1}$}

${ }^{1}$ Sydney South West Area Health Service, Biomedical Engineering

Background As they emerged from humble beginnings, as isolated technicians attached to clinical departments, biomedical departments have often grown by accretion. In the modern business, clinical and regulatory environment there is a need to review how departments are structured.

Method We reviewed several biomed departments in Australasia and examined structure in similar service organisations along with modern business structures. We then developed a generic model of a biomedical engineering department (Fig. 1) that incorporates many features currently lacking.

Results We found most departments had a simple one-dimensional structure consisting of, electronics, and mechanical sections, an admin assistant (or section), and a shallow hierarchy. In contrast, modern service organisations have a matrix (or cube) structure divided according to clinical and technical speciality as well as geography and functional level. In our new model there is a professional services section (technical leadership, policy, training, and regulatory management), a services section (do and charge, routine work), and an admin and management section (providing internal business functions).

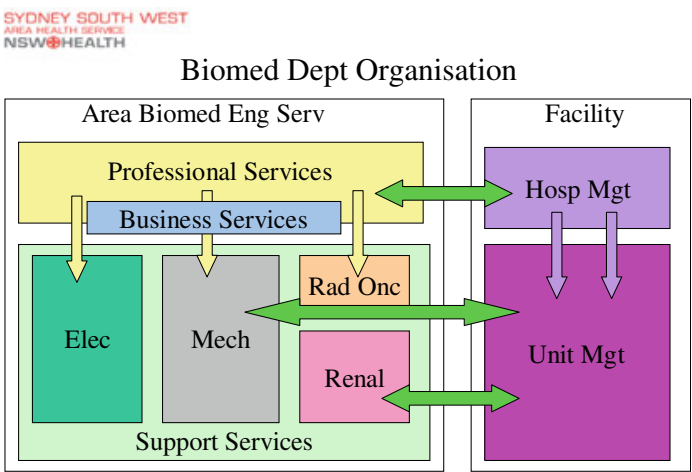

Fig. 1 A generic biomed department organisation. Professional services provide technical leadership and consultative services to facility senior management. Support services provide 'do and charge' services to ward/unit managers. Business services provide internal services. Technical streams are developed and lead by senior support services and professional services officers. This model can be expanded to include a second (matrix) dimension for services at multiple sites and can be refined to include clinical sub-specialities within a trade group (cube) to ensure the department meets the organisations needs with adequate levels of expertise 
We found the model provides flexibility to grow and adapt to future needs, levels of career progression, defined responsibility (for all sections). We believe such a model can be used to argue for better resourcing - through more clearly defined roles and responsibilities. By considering the whole spectrum of biomedical engineering services-not just the electronics and mechanical workshops-we believe this will result in a more professional looking organisation.

Conclusion A new departmental structure will provide a more consistent, professional, and focused department benefiting staff and facility alike.

\section{Session 12D—AS 3581 workshop}

Rob Wilkins ${ }^{1}$, Mike Flood ${ }^{2}$, Tony Blackler ${ }^{3}$, Adrian Richards ${ }^{4}$, Peter Taunton ${ }^{5}$

${ }^{1}$ Biomech, ${ }^{2}$ Therapeutic Goods Administration, ${ }^{3}$ Canterbury District Health Board, ${ }^{4}$ Women and Children's Hospital, ${ }^{5}$ Royal Children's Hospital

Abstract not available at time of printing.

\section{Session 13A-Radiotherapy 2}

\section{A 2009 survey of the Australasian clinical medical physics and biomedical engineering workforce}

\section{Howell Round ${ }^{1}$}

${ }^{1}$ Department of Engineering, University of Waikato, New Zealand

In 2006 a robust survey of the Australasian clinical medical physics and biomedical engineering workforce was carried out [1]. The survey showed where there were was an undersupply in the workforce and demonstrated that there was a need for resources to be put in to training and recruitment to overcome future shortfalls. Salary levels of those working in different disciplines, in different levels of experience and in different jurisdictions were also presented. The survey has proven to be a very useful tool for workforce planning.

The survey was repeated in 2009, and the results will be presented in this paper. Changes in the structure of the workforce can be seen and areas into which resources must be allocated to ensure a satisfactory future workforce are identified.

\section{Reference}

1. W H Round. A survey of the Australasian clinical medical physics and biomedical engineering workforce, Aust Phys Eng Sci Med, 30 (1), 13-24, 2007.

\section{Advances in targeted alpha therapy for metastatic melanoma}

\author{
Barry J Allen ${ }^{1}$ \\ ${ }^{1}$ Centre for Experimental Radiation Oncology, St George Hospital \\ Cancer Care Centre
}

Targeted alpha therapy (TAT) is a developing experimental cancer therapy based on the cytotoxic properties of the high linear energy transfer $(\sim 100 \mathrm{keV} / \mu \mathrm{m})$ and low range $(20-80 \mu \mathrm{m})$ of alpha particles. Monoclonal antibodies are labelled with alpha emitting radioisotopes to form the alpha-immunoconjugate (AIC), which can be highly lethal to targeted cancer cells but spare normal tissue.

Our research and development program for metastatic melanoma has passed from test tube to bedside. Phase 1 clinical trials have been completed for intralesional therapy ${ }^{1}$ and are well advanced for systemic therapy of metastatic melanoma ${ }^{2}$. A biological dosimeter ${ }^{3}$ has been developed based on the measurement of micronuclei in lymphocytes.

The melanoma systemic trial demonstrated that alpha therapy could regress solid tumours. This effect was ascribed to a process called Tumour Anti-Vascular Alpha Therapy (TAVAT) [4], which has the potential to change outcome in end-stage disease. Intravenous injections of up to $25 \mathrm{mCi}$ of the ${ }^{213} \mathrm{Bi}-9.2 .27$ alpha immunoconjugate have not caused any adverse events of any level. However, promising therapeutic responses have been observed in the 38 patients treated so far, being $10 \%$ partial response, $40 \%$ stable disease, and $10 \%$ long term survival at $2-5$ years.

\section{References}

1. Allen BJ, Raja C, Rizvi SMA, Li Y, Tsui W, Graham P, Thompson JF, Reisfeld RA, Kearsley JH, Morgenstern A, Apostolidis C. Intralesional targeted alpha therapy for metastatic melanoma. Cancer Biology Therapy, 2005: 4 (12); 1318-1324.

2. Raja C, Graham P, Rizvi SMA, Song E, Goldsmith H, Thompson J, Bosserhoff A, Morgenstern A, Apostolidis C, Kearsley JH, Reisfeld R, Allen BJ. Interim analysis of toxicity and response in Phase 1 trial of systemic targeted alpha therapy for metastatic melanoma. Cancer Biology \& Therapy. 2007 6:6, 846-52.

3. Song EY, Rizvi SMA, Raja C, Qu Changfa, Yuen J, Morgenstern A, Apostolidis C, Allen BJ. The cytokinesis-block assay as a biological dosimeter for targeted alpha therapy. Phys Med Biol. 2008; 53, 319-328.

4. Allen BJ, Raja C, Rizvi SMA, Song EY, Graham P. Tumour antivascular alpha therapy: a mechanism for the regression of solid tumours in metastatic cancer. Phys. Med. Biol. 52 (2007) L15L19.

\section{Session 13B-Telemedicine}

\section{Ten years of telepaediatrics—-lessons learnt in Queensland}

\section{Anthony Smith ${ }^{1}$}

${ }^{1}$ University of Queensland

In November 2000, the Centre for Online Health, (University of Queensland) commenced a research project aimed at establishing and evaluating a novel telepaediatric service in Queensland. ${ }^{1-3}$ The telepaediatric service is characterised by a centralised coordination unit, responsible for the facilitation of telepaediatric referrals.

The telepaediatric service has evolved into a major clinical support program at the Royal Children's Hospital-which directly benefits children, their families and the clinicians delivering services. More than 8500 consultations have been conducted for thousands of children in Queensland. The telepaediatric service covers 37 different sub-specialist areas and involves more than 240 medical, nursing and allied health staff. To date, telepaediatric services have been delivered to 94 regional hospitals in Queensland, several facilities in northern NSW, Western Australia and the Northern Territory.

The telepaediatric service has proven valuable for selected regional hospitals in Queensland. ${ }^{4}$ Our research has demonstrated substantial savings for the health department due to reduced patient travel ${ }^{5}$; benefits for regional families who are saved the inconvenience and costs of travel away from home ${ }^{6}$, improved communication and collaboration between regional hospital staff and specialists at tertiary hospitals; and 
valuable opportunities for professional education and support in remote areas which traditionally are underserved ${ }^{7}$.

This presentation will provide a broad overview of telepaediatrics in Queensland. Aspects such as feasibility and cost-effectiveness will be addressed.

\section{References}

1. Smith AC. The feasibility and cost-effectiveness of a novel telepaediatric service in Queensland. The University of Queensland, Australia, November 2004. URL: http://adt.library.uq.edu.au/ public/adt-QU20050121.145040/index.html.

2. Smith AC, Isles A, McCrossin R, Van der Westhuyzen J, Williams M, Woollett $\mathrm{H}$ and Wootton R. The point of referral barrier-a factor in the success of telehealth. Journal of Telemedicine and Telecare 2001; 7 (Suppl.2): 75-783.

3. Smith AC. Telepaediatrics. Journal of Telemedicine and Telecare, 2007, 13 (4): 163-166.

4. Smith AC and Gray LC. Telemedicine across the ages. The Medical Journal of Australia. 2009, 190 (1): 15-19.

5. Smith AC, Scuffham $P$ and Wootton R. The costs and potential savings of a novel telepaediatric service in Queensland. BMC Health Services Research 2007, 7:35, available online at http://www.biomedcentral.com/1472-6963/7/35.

6. Smith AC, Youngberry K, Isles A, Mc Crossin R, Christie F and Wootton R. The family costs of attending hospital outpatient appointments via videoconference and in person. Journal of Telemedicine and Telecare 2003; 9 (Suppl. 2): 58-61.

7. Harris V, Smith AC and Armfield N. e-Learning for regional health professionals using mobile videoconferencing in Queensland. Journal of Telemedicine and Telecare, 2007, 13 (Suppl. 3): 44-47.

\section{Telemedicine developments from CSIRO}

\section{Chris Gunn ${ }^{1}$}

${ }^{1}$ Immersive Environments Laboratory, CSIRO ICT Centre

I will present an overview of telehealth technology developed by the CSIRO ICT Centre. It covers three systems:

- VICCU-Virtual Intensive and Critical Care Unit: a trolley that can be wheeled up to an intensive care patient's bed and acts as a surrogate for a doctor monitoring and advising from a distant location.

- Echonet: a link between two hospitals for viewing live echocardiograph output.

- RIDES-Remote Immersive Diagnostic Examination System: clinic-to-specialist patient consultation system enabling remote and rural patients to be examined and consulted by a doctor or specialist in the city.

Most of the talk will be focused on the RIDES system as I was involved in its development.

\section{Session 14A-Advanced treatment technologies}

\section{Volumetric modulated arc radiotherapy with smartArc: comparison of smartArc and conventional IMRT for prostate radiotherapy}

Nicholas Hardcastle ${ }^{1}$, Wolfgang Tomé ${ }^{2}$, Kerwyn Foo ${ }^{1,3,4}$, Andrew Miller $^{3,4}$, Martin Carolan ${ }^{3}$, Peter Metcalfe ${ }^{1}$

${ }^{1}$ Centre for Medical Radiation Physics, University of Wollongong, ${ }^{2}$ Department of Human Oncology and Medical Physics, University of Wisconsin, USA, ${ }^{3}$ Illawarra Cancer Care Centre, Wollongong Hospital, ${ }^{4}$ Graduate School of Medicine, University of Wollongong
Purpose To compare conventional IMRT plans with Volumetric Modulated Arc Radiotherapy plans for prostate radiotherapy.

Methods and materials The smartArc Volumetric Modulated Arc Radiotherapy optimisation tool in the Pinnacle Treatment Planning System (TPS) was used to create volumetric modulated arc therapy plans for ten prostate patient using biological objectives. A seven field conventional IMRT plan using the same optimisation objectives was created for each patient for comparison. The target and organ at risk doses were compared as well as the rectal NTCP using three sets of Lyman-Kutcher-Burman (LKB) model parameters (NTCP1: $n=0.24$, $m=0.14, \quad \mathrm{D} 50=75.7 \mathrm{~Gy}$ and NTCP2: $n=1.03, \quad m=0.16$, $\mathrm{D} 50=55.9 \mathrm{~Gy}$ ). The delivery efficiency was compared using required monitor units and expected treatment time.

Results The smartArc plans resulted in lower rectal volumes receiving doses $<45$ Gy for equivalent target coverage. Rectal volumes receiving $>45$ Gy were approximately equal for both techniques. The smartArc plans resulted in a lower rectal NTCP for parameter set NTCP2 (0.91\% (IMRT) versus $0.55 \%$ (smartArc), $P<0.01$ ) but no statistically significant difference for parameter set NTCP1 was observed. The delivery efficiency was improved with smartArc, with on average $18 \%$ fewer monitor units required. Average delivery time was reduced from $7 \mathrm{~min} 30 \mathrm{~s}$ to $2 \mathrm{~min}$ with smartArc.

Conclusion The smartArc plans resulted in reduction in rectal volumes receiving mid-low doses for equivalent target coverage. This lead to a statistically significant reduction in rectal NTCP for one set of LKB model parameters. The advantages of reduced rectal dose were supplemented by a reduction in required monitor units and significant reduction in delivery time.

\section{Implementation of two-step intensity modulated arc therapy}

Jidi Sun ${ }^{1}$, Juergen Meyer ${ }^{1}$, Theam Yong Chew ${ }^{2}$

${ }^{1}$ Department of Physics and Astronomy, University of Canterbury, New Zealand, ${ }^{2}$ Lincoln Ventures Ltd, New Zealand

Introduction Intensity-modulated arc therapy (IMAT) allows creating highly conformal treatment plans, which are supposedly superior to conventional intensity-modulated radiation therapy in terms of treatment time and dose conformity [1]. However, planning is complex and the relative benefits are not yet fully understood [2]. In this work we describe the implementation of a simplified technique called 2-step IMAT, which requires two rotations of the gantry [3]. The beam segments for the first rotation are in analogy with a conformal arc, i.e. with one aperture for each gantry angle reflecting the shape of the target volume. The beam segments for the second rotation are narrow segments close to the organ-at-risk (OAR) in order to achieve steep dose gradients on the edge of the tumour adjacent to OARs.

Methods and materials Two-step IMAT was implemented into the University of Washington treatment planning system Prism using the Common Lisp Programming language. The segments are automatically generated using 3D-anatomy information and ray-tracing. An elliptical phantom was created with a concave planning target volume (PTV) and an OAR. The width of the narrow segments as well as its relative weight was varied in order to optimise the plan for the phantom. A comparison was carried out between a 4-field 3D-conformal plan, conformal arc plan and 2-step IMAT.

Results/discussion The two-step IMAT plan showed the potential of significantly sparing the OAR with regards to the other techniques (see Fig. 1). However, the target dose uniformity was not within the $-5 \% /+7 \%$ criteria. The slight dose non-uniformity in the target was not unexpected since no beam weight adjustments had been made for the different pathlengths from different gantry angles. 


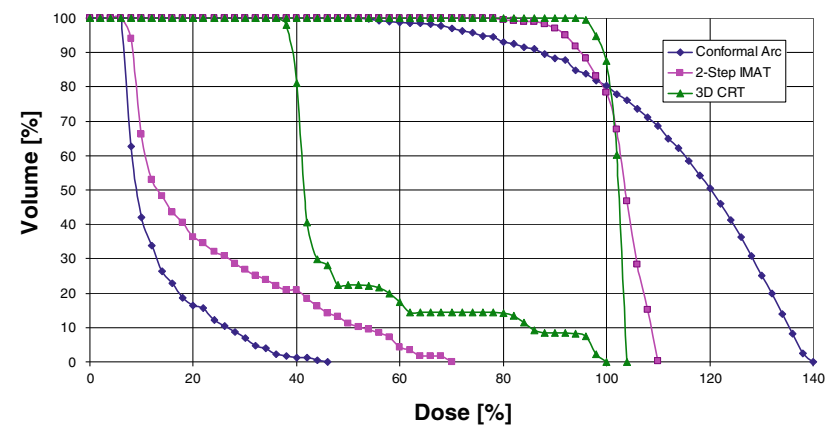

Fig. $1 \mathrm{DVH}$ for 3D conformal (triangle), single arc (diamond) and 2-step IMAT (square)

Conclusion In this proof-of-principle study we have for the first time automated and implemented the process of segment generation for 2-step IMAT and initial results indicated that with this technique it is possible to create highly conformal treatment plans with high dose gradients. To overcome the current limitations implementation of dose-volume constraint based optimisation and further investigations on the effects of beam aperture settings are required.

\section{References}

1. Yu C X. Intensity-modulated Arc Therapy with Dynamic Multileaf Collimation: An Alternative to Tomotherapy. Physics in Medicine and Biology 1995; 40:1435-1449.

2. Bortfeld T, Webb S. Reply to 'Comments on Single-Arc IMRT?' Physics in Medicine and Biology 2009, 54; L35-L36.

3. Bratengeier K. Applications of Two-Step Intensity Modulated Arc Therapy. Strahlentherapie Und Onkologie 2001; 177:394403.

\section{The commissioning and implementation of VMAT at NCCI}

\section{Brett Waller}

\section{${ }^{1}$ North Coast Cancer Institute}

Intensity modulation arc therapy (IMAT) is an enhancement of existing technology which offers a number of potential treatment delivery advantages over IMRT and 3D conformal treatments. The implementation of IMAT technology offered by Elekta is known as volumetric modulation arc therapy (VMAT) and is currently undergoing clinical implementation at the North Coast Cancer Institute (NCCI) in NSW. VMAT has presented both potential benefits and challenges in terms of commissioning and quality assurance.

The evaluation and implementation of VMAT as a clinical treatment modality involved a multidisciplinary team effort and a process which required a number of steps:

Initial justifications for implementation which include:

- an alternative available for cases where using existing conformal and IMRT techniques were difficult to plan;

- potentially quicker delivery times which could reduce infrafraction motion, patient discomfort and anxiety;

- a possible reduction of monitor units required for delivery;

- less uncertainty associated with small-segment IMRT dosimetry.

Establishing an implementation methodology, i.e.

- acquisition and commissioning of a VMAT-capable inverse planning system;

- validation method used for testing the planning system based on TRS-430;
- retrospective plan comparisons with plans using current delivery techniques (conformal and IMRT).

Configuration of equipment and treatment delivery validation, i.e.

- upgrade of treatment equipment to enable VMAT delivery;

- validation of clinical VMAT plans and delivery;

- QA considerations and on-going development of QA methods.

The results of clinical implementation together with a review of real benefits that have been gained will be presented.

Investigation of bulk electron density planning and automatic bone segmentation on T2* MR images for MR-based prostate radiotherapy planning

Jonathan Lambert ${ }^{1}$, Jason Dowling ${ }^{3}$, Olivier Salvado ${ }^{3}$, Jackie Patterson $^{2}$, Fred Menk ${ }^{1}$, Peter Greer ${ }^{1,2}$

${ }^{1}$ University of Newcastle, ${ }^{2}$ Calvary Mater Hospital, ${ }^{3}$ CSIRO, Australian E-Health Research Centre

Introduction The major factor hindering the implementation of MR-based prostate radiotherapy planning is the lack of electron density information in the scans. An appropriate density for the bone needs to be calculated, and applied to the bone in the MR images. Previous investigations of MR based planning have had very small patient sample sizes. In prostate radiotherapy, T2* MR images are used to image the gold seed markers implanted in prostate cancer patients, and these images also display bone as darker than surrounding tissues. They may therefore prove to be useful for automatic bone segmentation.

Methods Existing treatment plans for 39 patients who had all previously completed treatment were used to create bulk electron density plans. These plans were based on the CT images, and plans both with and without bone (i.e. a completely homogenous density) were created. An appropriate bulk electron density for bone was calculated using effective depth calculations. For any patients that were treated over more than one phase, each phase was considered separately to account for any differences in the treatment. A pilot study using T2* MR images from three prostate cancer patients was also carried out to investigate automatic MR bone segmentation. The images were imported into MeVisLab [1] and filtered to improve the contrast between the bone and other tissues. Two ramp filters were used (acting in the positive $x$ and $y$ directions), followed by a windowing filter. Bone segmentations were created in ITK-Snap [2] using the filtered images and using the standard spherical seed points. The seed points were grown to the image edges of the pre-processed images. For one of the image sets a mask was applied to isolate the bone and the result was also filtered in MeVisLab. Segmentations were created in MeVisLab based on these images, and the results used as starting points in ITK-Snap.

Results The density of bone was calculated to be $1.19 \mathrm{~g} / \mathrm{cm}^{3}$. Using this value, CT-based bulk electron density plans with bone differed by a mean of $0.1 \%$ when compared to the intended delivery of $200 \mathrm{cGy}$ on the full density CT plan, with a standard deviation of $0.6 \%$. Bulk electron density plans that assumed a completely homogenous, water equivalent density differed by a mean of $1.4 \%$, with a standard deviation of $0.8 \%$. The segmentations produced on the filtered images include most of the bone in the inner slices, but only segments of the bone in the outer slices. Using a mask and a variety of filtered images to aid segmentation provides the most consistent results.

Discussion The good agreement between the CT-based bulk electron density plans that include bone suggest that the calculated value of $1.19 \mathrm{~g} / \mathrm{cm}^{3}$ is a suitable value to use. Future work will involve applying this value to MR-based bulk electron density plans with 
manually defined bone contours. However, due to the different geometry of anatomy in the MR images, the difference between MRbased bulk electron density plans with bone may result in a larger difference when compared to the full density plans. The prospect of automatic bone segmentation on MR images is also promising. While there are problems segmenting the bone in the outer slices, this is most likely due to the algorithms used in ITK-Snap, rather than the quality of the images.

Conclusion MR-based treatment planning of prostate cancer using bulk electron densities is feasible. Future work will involve applying bulk electron densities and treatment plans to the MR images of each patient using manual bone contours. The results suggest that it may be feasible to automatically segment bone using T2* MR images, but a standard procedure is yet to be established.

Acknowledgments This work was partially funded by Cancer Council NSW Grant Number RG 07-06.

\section{References}

1. MeVis Medical Solutions AG. 'MeVisLab-Medical Image Processing and Visualization' 2006, Accessed 11/6/2009; Available from: http://www.mevislab.de.

2. Yushkevich, P. A., J. Piven, H. C. Hazlett, R. G. Smith, S. Ho, J. C. Gee, and G. Gerig, 'User-guided 3D active contour segmentation of anatomical structures: Significantly improved efficiency and reliability,' NeuroImage, 2006. 31: p. 1116-1128.

\section{B-Research and developments in medical physics}

\section{Is targeted anti-vascular alpha therapy efficacious and safe?}

\author{
Chen-Yu Huang ${ }^{1,2}$, Bradley Oborn ${ }^{3}$, Barry J Allen ${ }^{1,2}$ \\ ${ }^{1}$ Centre for Experimental Radiation Oncology, St George Hospital \\ Cancer Care Centre, ${ }^{2}$ St George Clinical School, University of New \\ South Wales, ${ }^{3}$ Centre for Medical Radiation Physics, University of \\ Wollongong
}

Targeted anti-vascular alpha therapy (TAVAT) causes effective tumour regression by killing endothelial cells in tumour capillaries. A Geant4 Monte Carlo simulation has been adapted to simulate the spatial non-uniform distribution of the radioisotope source ${ }^{213} \mathrm{Bi}$ and to investigate the microdosimetry in a capillary endothelial cell model.

Background, luminal and perivascular geometries were evaluated with the source in the following geometries: source inside an $8 \mu \mathrm{m}$ diameter, $50 \mu \mathrm{m}$ long capillary; on the endothelial cell intraluminal membrane and on membranes of cancer cells and capillary pericytes respectively.

The average probability of alpha particles hitting the endothelial cell nucleus in the above three geometries was $0.5,0.6$ and 0.2 , respectively. The specific energy per hit was $2.7,2.6$ and $2.5 \mathrm{~Gy}$ and $1.3,1.6$ and 0.5 cGy correspondingly per ${ }^{213} \mathrm{Bi}$ decay.

In our clinical trial, up to $25 \mathrm{mCi}$ of ${ }^{213} \mathrm{Bi}$ radioimmunoconjugates (RIC) with specific activity in $3.2 \mathrm{mCi} / \mathrm{mg}$ was used for systemic injection and the labelled fraction is 1:5000. For $10^{5}$ membrane antigens and 40 cancer cells and capillary pericytes around one capillary endothelial cell, there would be $\sim 800$ RICs in the perivascular space. This could give up to $4.2 \mathrm{~Gy}$ to the endothelial cell nucleus or 21 RBE.Gy using RBE equals to 5 for alpha radiation compared with the background dose of $1.3 \mathrm{cGy}$ and 6.5 RBE.cGy $\sim 99 \%$ survival rate for normal capillary endothelial cell.
These results show that TAVAT holds promise to deliver a lethal dose to the tumour capillary endothelial cells while preserving the function of normal capillaries.

\section{Dose measurement and enhancement of radiotherapeutic type proton beams}

\author{
Pradip Deb ${ }^{1}$, Christopher Wong ${ }^{1}$, Moshi Geso ${ }^{1}$ \\ ${ }^{1}$ Discipline of Medical Radiations, School of Medical Sciences, \\ RMIT University
}

Introduction Proton radiotherapy is increasingly becoming an attractive procedure for cancer treatment. Its main advantage is the uniqueness of the dose distribution. Therefore, it is vital to determine the dose deposited by protons in tissue equivalent material and also to increase the effectiveness of such a dose. In this work, $230 \mathrm{MeV}$ proton beam was used and three dimensional dose distribution was determined employing polymer gels. The dose was enhanced using gold nanoparticles (AuNPs) embedded in the target.

Method $230 \mathrm{MeV}$ proton beam was obtained from National Cancer Centre, Korea. The beam was passed through layers of Perspex in order to ascertain the placement of polymer gels in the spread out Bragg peak (SOBP) of uniform dose distribution. The procedure was repeated for various concentrations of AuNPs (0.5-1.5 mM with $2 \mathrm{~nm}$ particle size). The irradiated polymer gels were scanned in Alfred Hospital, Melbourne, using 3T MRI scanner.

Results The SOBP was obtained and validated against the measurements of ionisation chamber and gafchromic films. Agreement between polymer gels and films is about 5\%. The effect of nanoparticles is found to be augmenting the effectiveness of the proton dose. This effect is observed to be linearly related to the AuNPs concentrations. However it should be noted that the AuNPs were activated during radiation via proton-gold interactions.

Conclusion Previously has been shown limited use of polymer gels for proton at low energies. Here this has been extended to the high energy beams and modified structure of gels to suit proton beam dosimetry. The dose can be perturbed with the inclusion of higher concentration of AuNPs to enhance the effects of proton-nucleus reactions.

\section{Amelioration of light scattering artefacts in optical CT for polymer gel dosimetry}

Stephen G. Bosi ${ }^{1}$, Sofie Ceberg ${ }^{2,3}$, Tina Gorjiara, Justin Davies ${ }^{4}$, Helen Gustavsson ${ }^{2,3}$, Paul White ${ }^{1}$, Clive Baldock ${ }^{2}$

${ }^{1}$ Prince of Wales Hospital, ${ }^{2}$ University of Sydney, ${ }^{3}$ Malmo University Hospital, ${ }^{4}$ Nuclear Science \& Technology Organisation, Lucas Heights, NSW Australia

Introduction Dosimetric polymer gels, (e.g. PAGAT [1]) increase optical density (OD) with dose. Gels can be read using Cone Beam optical CT (CBopCT). Attenuation via scattering produces stray light. CBopCT is susceptible to scattered light artefacts, limiting gel dosimeter accuracy and dynamic range [2]. Susceptibility to scattering is reduced by: (1) reducing the scanner camera aperture; (2) fitting polaroid filters.

Method Polymer gel dose was simulated using Dettol ${ }^{\mathrm{TM}}$ in gelatin [2]. A phantom was produced, containing a funnel-shaped, high OD region surrounded by an outer, low OD region. It was scanned with a Modus Vista Optical CT Scanner and reconstructed (Feldkamp backprojection). Horizontal OD profiles through the funnel were obtained from the reconstruction. 
For Method (1) scans were performed using a range of camera aperture sizes.

For Method (2) a polaroid filter (polariser) fitted over the scanner light source and polaroid analyser mounted before the camera lens. Various configurations of polariser and analyser angle were examined.

Results and discussion Ideal OD profiles should be plateaus, reflecting the uniform Dettol concentration. Actual profiles exhibited 'dishing' artefacts. The measured optical density profile dips downwards near the profile centre (Fig. 1), an effect of scattered stray light [2].

Reducing the camera aperture from size 1-4 decreased the severity of dishing at the expense of signal intensity (Fig. 1).

Certain polaroid configurations reduced dishing, but introduced other artefacts (Fig. 1)

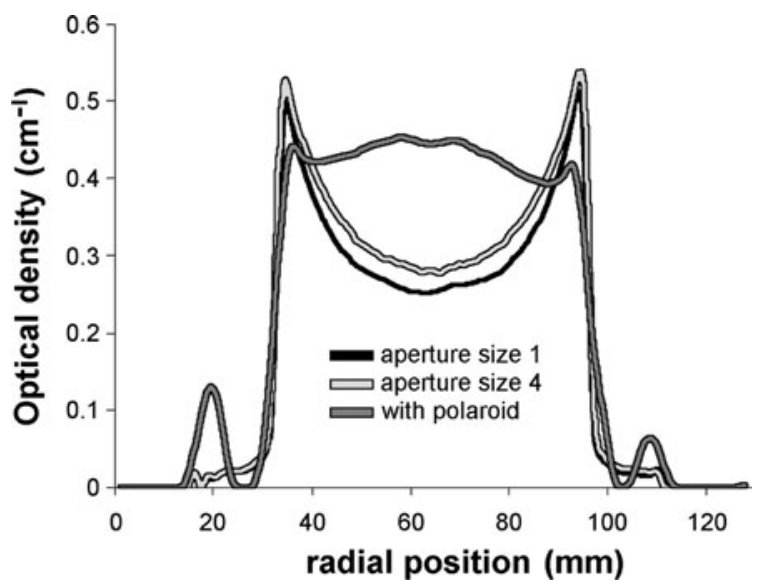

Fig. 1

\section{References}

1. A.J. Venning et al. PMB $\mathbf{5 0} 3875-3888$ (2005).

2. S.G. Bosi et al. PMB 54 275-283 (2009).

The radiobiology of intra-fractional repair: theoretical and experimental evidence for temporal dose pattern effects

\author{
N Suchowerska ${ }^{1,2}$, DR McKenzie ${ }^{1}$, JM Bewes $^{1}$, M Ebert $^{3,4}$ \\ ${ }^{1}$ School of Physics, The University of Sydney, ${ }^{2}$ Department of \\ Radiation Oncology, Royal Prince Alfred Hospital, ${ }^{3}$ Department of \\ Radiation Oncology, Sir Charles Gairdner Hospital, ${ }^{4}$ School of \\ Physics, University of Western Australia
}

Aim The effects of temporal modulation of a course of radiotherapy are well documented and are used to determine fractionation schedules. The effect of temporal modulation within a treatment fraction is not as well understood. Taking a simplistic approach, radiation can interact with tissue creating either a lethal lesion (e.g. double strand break) or a sub-lethal lesion (e.g. single strand break). Using a formulism derived from the Linear-Quadratic (LQ) model, which includes a dose protraction factor, the effects of temporal modulation in protracted radiotherapy treatments, can be determined. The aim of this study is to identify the optimal temporal dose pattern to either maximise or minimise cell survival for a given dose. The significance of this work is that it offers an alternative approach to maximising the therapeutic ratio of a treatment without changing the dose.
Method The cytotoxic effects of several intra-fractional temporal dose patterns were determined theoretically and experimentally. The theoretical survival fraction was calculated using the Linear Quadratic formulism, which includes a Dose Protraction Factor (Lea and Catchside, 1943), as given below:

$G=\left(\frac{2}{D^{2}}\right) \int_{0}^{t} R(t) \mathrm{d} t \int_{0}^{t} e^{-\lambda\left(t-t^{\prime}\right)} R\left(t^{\prime}\right) \mathrm{d} t^{\prime}$

The experimental survival fraction was determined using two cancer cell lines: non-small cell lung (NCI-H460) and melanoma (MM576). Each cell line was exposed separately to several different dose delivery patterns, keeping the overall treatment time constant for each exposure. Clonogenic survival fractions were derived from at least 9 replicates for each experimental condition. Data uncertainties are presented as the standard error of the mean (SE).

Results and discussion MM576 cells exposed to 6 Gy exhibited a significantly different survival fraction when exposed to different temporal patterns in a treatment fraction, keeping the dose constant and the total treatment time at $20 \mathrm{~min}$. However a statistically significant difference in response to different temporal dose patterns was not found for the NCI-H460 cell line. This result is consistent with the theoretical predictions using the modified Linear Quadratic Model, including the Protraction Factor and indicates that the intra-fractional temporal effect may vary depending on the cells' $\alpha / \beta$ ratio. Since cells with a low $\alpha / \beta$ ratio show a greater sensitivity to temporal modulation effects, temporal modulation may be a vehicle by which normal tissue can be spared with no alteration to the dose distribution. Furthermore, tumours with low $\alpha / \beta$ ratios such as prostate cancer are particularly susceptible to changes in temporal modulation and may be ideally suited to temporal optimisation.

Conclusion Intensity-modulated radiation therapy (IMRT) and scanned proton pencil beam technology offer an opportunity to customise the temporal dose delivery pattern for highest cytotoxicity.

Acknowledgments The authors acknowledge funding from the NSW Cancer Council in support of this research.

\section{Session 14C-Biomedical engineering}

\section{Technology embedment structure in medical setup of Pakistan}

\author{
Maria Hafeez ${ }^{1}$, Muhammad Muneeb Shaukat ${ }^{1}$ \\ ${ }^{1}$ National Institute of Electronics, Ministry of Science and \\ Technology, Pakistan
}

This paper aims to picture the enhanced NADRA (National Database and Registration Authority) Kiosk Services in Health and related sectors of Pakistan. Using the existing data base of NADRA an e-health system can be developed, which is equally usable and accessible in rural and urban areas of the country. Mainly this service aims to provide a smooth platform towards E-Prescription. This can also prove to be a check and balance to control the improper usage of drugs in new generation specifically.

E-Prescription design will be based upon modular design application. An e-Health server will be used as bridge between Kiosk machine and doctor machine. Patient has to swap his/her NADRA CNIC (computerised national identification card) card along with biometric identification. CNIC will be sent to NADRA data base and basic information and history of the patient will be displayed to doctor. Doctor will give him an online E-prescription which will be saved against his CNIC card number; this E-Prescription will be accessible by authorised drug centre. 
This research will help to control the unauthorised non subscribed misuse of drugs. Doctors will have the case history of patients. Monitoring of patients, doctors and drug centres will be transparent helping the country in case of taxation also.

The proposed model is practically implementable and can be useful for patients and doctors and for the economical growth of the country.

\section{Remote vital signs monitoring for the Cooeenet@qld Project}

\section{Phil Cole ${ }^{1}$}

\section{${ }^{1}$ Queensland Health}

Telehealth offers a potential solution to the tyranny of distance which affects equity of health care in rural and remote locations in Queensland. Under the Commonwealth's Clever Networks, Innovative Service Delivery program, funding was provided to implement remote vital signs monitoring equipment to supplement existing Telehealth videoconferencing equipment. The primary use of this is for aiding in the management and coordination of emergency retrievals. Issues and experiences encountered during the procurement and installation will be discussed, including system architectures, interoperability and standards compliance, GITC, interaction with ICT staff, networking, installation and clinical outcomes.

\section{Development of an ambulatory multi-parameter physiological monitoring system}

\section{Ke Ma ${ }^{1}$, Joshua Khoo ${ }^{1}$, Ian Brown ${ }^{1}$}

${ }^{1}$ Department of Electrical and Computer Systems Engineering, Monash University

Long-term stress is considered as the cause of various chronic diseases, such as cardiovascular diseases and depressiondrpreann. It may also have a detrimental effect on human performance of complex cognitive and motor tasks. A novel ambulatory multiparameters monitoring system has been developed for providing long term stress related physiological parameters monitoring. This system is able to measure cardiovascular system, respiration system, temperature regulation and activity related parameters. System validation studies have been undertaken in the laboratory and the physiological changes during emotional stress and activity were detected effectively.

The monitoring system consists of a body worn sensor unit, a mobile phone and a remote server, as Fig. 1 showing. The body wear unit is designed as a $40 \mathrm{~mm} \times 47 \mathrm{~mm}$ compact patch integrating the sensors, containing the signal conditioning circuit, battery and the Bluetooth telemetry unit. A Body Area Network (BAN) uses Bluetooth to transmit data to a mobile phone, as Fig. 2 showing. The limited computation power has been used to implement low level of digital signal processing on raw digitalised signal. A $3.7 \mathrm{~V}$ Lithium ion polymer battery (290mAh) has been employed to power the signal conditioning circuit and the Bluetooth telemetry unit. The mobile phone sends the data via internet to the remote station. Patients can be monitored within the mobile phone catchment area anywhere in the world.

The system has been validated in the laboratory and the tested physiological parameters detected by this system correspond to the emotional states, as Fig. 3 showing. It is intended to further validate the system by conducting studies of stress in physicians undergoing anaesthesia simulator training.

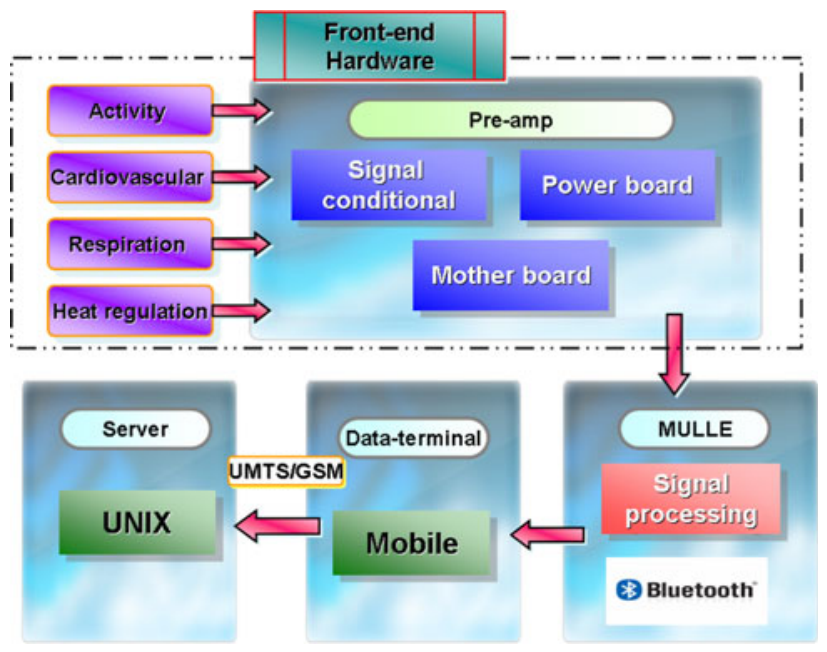

Fig. 1
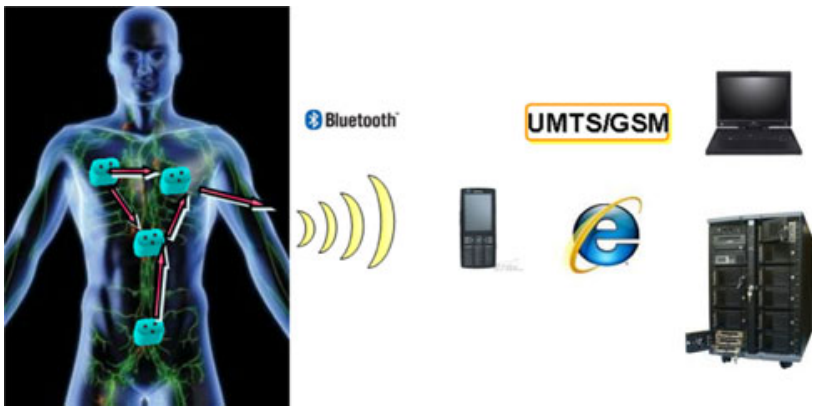

Fig. 2
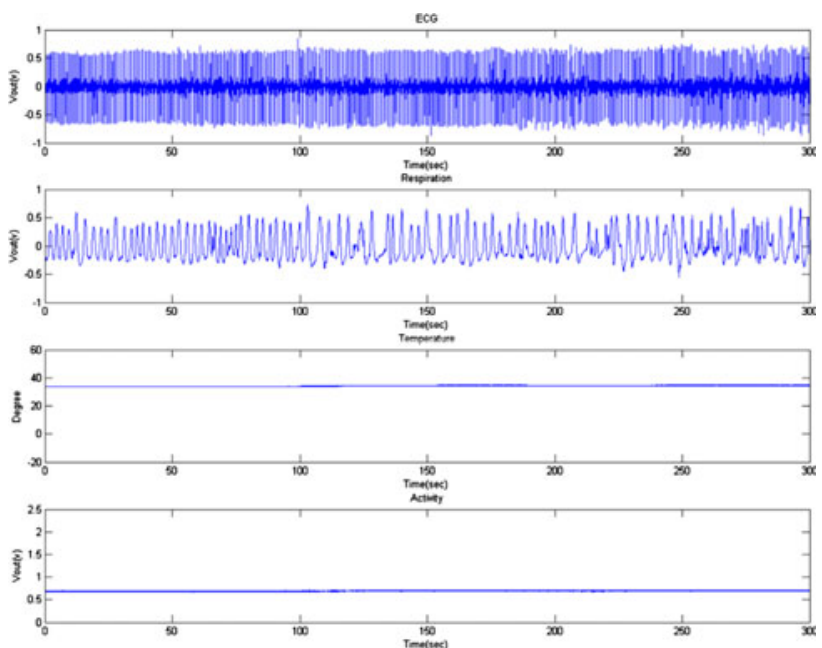

Fig. 3 


\section{Session 14D-Working in remote areas}

\section{Working remotely in developing countries-keeping in touch and working effectively}

\author{
Bruce Morrison $^{1}$, Stanley Scahill ${ }^{2}$ \\ ${ }^{1}$ Haniham Pty Limited, ${ }^{2}$ Sydney South West Area Health Service
}

Working remotely in developing countries involves not only isolation from one's family and colleagues but also from sources of information. It usually means there will be delays in getting needed resources to be effective in the job and so strategies need to be investigated to overcome these obstacles.

Sources of frustration include communication difficulties (speed, fidelity), communications costs, working in different time zones, limited access to service information, uncertain financing of spare parts, uncertain deliveries of goods, limited access to peer networks for assistance and the general isolation of working away from home.

Other frustrations include old equipment or equipment from nontraditional suppliers. Tacking such equipment usually means that the likelihood of obtaining spares and/or service information is negligible so innovation is required and there is a need to think outside the square. Another thing to be aware of when working remotely in developing countries is that the engineer or technician will need to be multi-skilled to allow him or her to repair emergency generators, switchboards, solar hot water systems, incinerators, water pumps and sterilisers.

This paper explores some of the strategies and systems that may help to alleviate some of these difficulties. Overseas aid is a career satisfying experience so we encourage more biomedical engineers and technicians to participate.

\section{MAMCI Project-medical aid mission for communities in need}

\section{Jason Almeida ${ }^{1}$}

${ }^{1}$ Queensland University of Technology

This is a presentation on our project from start to finish, detailing the issues, the successes and the failures.

The MACMCI project was conceived in 2006. The idea was expressed in one sentence by Achi Kushnir the MAMCI Project Coordinator (2006-2007) 'M.A.M.C.I (Medical Aid Mission for Communities In need) is a student run project that aims set up a health centre by collecting redundant medical equipment from hospitals around Queensland, maintaining, repairing and distributing it along with spare parts, knowledge and training programs to communities'. The health centres in these areas have little to no equipment of supplies nor do they have electricity or proper plumbing. The system is not functioning and the rural people pay with their well being.

The project took three QUT students on a tour of the East Sepik Region and to deliver the goods to the health centres. These students, prior to the trip, handled the equipment and prepared it for arrival while engaging with Brisbane's Greenslopes Private Hospital and Academic Staff at QUT to get to know as much as they could about the donated goods. Upon receiving the goods at the other end the health centre staff were gathered and shown how to use and maintain the beds, with what they had available to them.

The students faced many difficulties on the trip. Cultural influences shaped the flow of the project while lack of supplies and equipment meant that the students had to look into alternate ways of maintaining the goods in working order. While the students could suggest that the health centres buy an item or get some supplies, lack of money and bureaucracy stands in the way.
The project would not have been possible if it were not for correspondence with FOWIAD (Foundation Of Women In Agricultural Development), an NGO working in the area. FOWIAD assisted in the organisation and translation of our ideas within the Sepik Culture. Not only did they assist the project before and during the trip, but as part of the sustainability of the project FOWIAD will be checking up on the condition and use of the donations every 3-6 months. The information collected will then be sent back to the team here to ensure that if there are problems, an appropriate solution can be devised and implemented on the ground.

The students were in Papua New Guinea for three weeks and learnt invaluable lessons. The recipients of our donated medical goods were so glad to have actually have received a donation like this that they attended as much of our demonstrations and presentations as possible. They expressed a high level of interest and showed promise of continuing what we had shown them and talked to them about. The enthusiasm and attendance meant that they were able develop with our assistance a way for them to handle what they had learned in a way that they would be able to continue use for years to come.

\section{Health of biomedical staff and nosocomial infections from medical devices}

\author{
Anne-Louise Smith ${ }^{1,2}$ \\ ${ }^{1}$ Biomedical Engineering, Flinders Medical Centre, \\ ${ }^{2}$ School of Medicine, Flinders University
}

Are biomedical staff placing themselves at risk by servicing general biomedical devices?

While care is taken with devices known to be used with infectious patients (e.g. HepB), few precautions are used by technical staff when servicing and testing general medical devices.

Hospital acquired infections affect about $6 \%$ of patients. Many micro-organisms are dangerous to immune-compromised patients and have easy access to the body by wounds and catheters.

Biomedical staff are generally healthy, and do not have easy microbial access to the body, except when injured while working, for example, slipping with a screwdriver resulting in a stab to the hand.

It may not be common knowledge to biomedical staff that some micro-organisms are able to stay viable in dry conditions, on nonporous surfaces with no enrichment. Some examples are: acinetobacter species 1-5 months, methicillin-resistant staphylococcus aureus (MRSA) 38 weeks on packaging, vancomycin-resistant enterococcus (VRE) 7 days to 4 months on dry surface.

Method A literature review was conducted on the microbial population of medical devices. To compare this with local conditions, a small microbial survey was undertaken of the control panels a random selection of 15 portable medical devices sitting in the Biomedical Engineering Department at Flinders Medical Centre.

Results The literature review showed many portable devices commonly found near patients are known to have microbial contamination. The device survey showed scanty growth only of species commonly found on hands.

Conclusion Although the literature shows microbial contamination of medical devices to be common, the results from our survey show low risk in our hospital.

It is recommended that biomedical staff follow good infection control practices: timely washing of hands throughout the day, alcohol handwash at all benches, not eating food at the workbench, and wiping devices with a damp cloth before working on them. 


\section{Engineers Without Borders}

\section{Huy Nyugen ${ }^{1}$, Simon Osenieks ${ }^{1}$ \\ ${ }^{1}$ Engineers Without Borders Australia}

Engineers Without Borders Australia (EWB) is a not-for-profit member driven organisation who works with disadvantaged and developing communities to improve their quality of life through appropriate technology, sustainable engineering and education. EWB is currently working with communities in countries including India, Nepal, Cambodia and Vietnam, and with Indigenous Australia. EWB works through long-term partnerships with in-community organisations to provide specific expertise, capacity building and facilitation as identified by the partner, delivered by volunteers who spend from 3 to 12 months working in-community. This is across six key themes, Information and Communication Technologies (ICT), Water and Sanitation (WatSan), agriculture, small-scale infrastructure, disability access and education.

EWB is currently working with both the Cambodia and Sri Lankan School's of Prosthetics and Orthotics, who train men and women to support and rehabilitate people with physical disabilities including manufacturing and fitting prosthetic limbs and orthopaedic braces. EWB works to develop, and assists in delivering, teaching material for their internal training and bridging programs. EWB also has two volunteers at KSE Medical in Vietnam, helping to develop a new neonatal medical device.

EWB ACT Chapter members Huy Nyugen and Simon Osenieks will provide a brief overview of EWB, its approach to development and its current programs and learning and change initiatives. EWB's work with the School's of Prosthetics and KSE Medical will also be provided as examples of EWB's work in different communities, in addition to a recent research project on an approach to disability inclusiveness in engineering development.

\section{POSTERS}

\section{Biomedical engineering}

\section{Support surface selection for acute phase spinal cord injuries} treatment

\section{Ian Craig $^{1}$}

${ }^{1}$ Hunter New England Clinical Technology

Spinal cord injuries are difficult injuries to treat due to complications arising from dealing with injuries to the spine and central nervous system whilst treating the often associated trauma to internal organs. At present 'There is no standardised approach to the clearance of the cervical spine in intubated trauma patients in Australian intensive care units. In addition, morbidity from current practices and the true incidence of cervical spine injuries remains unknown ... A standardised approach to radiological and clinical clearance of cervical spine injuries, which is practical, yet safe, remains a subject for future risk-benefit analysis'. (D Lien, 2003; 5)

The purpose of our study was to investigate many of the impacts of current spinal cord injury treatment surrounding the greatest cause of morbidity in such patients-the development of pressure ulcer developments during treatment in the acute phase (intensive care). It focused primarily on the role of support surface selection and patient positioning to the prevention of decubitus ulcers and upon pressure redistribution. Using the work of Seiler and Stahelin (Seiler WO, 1979) as a basis of our study we were able to estimate the likelihood of a patient to developing a decubitus pressure ulcer using a range of physical characteristics as a means of comparison.

\section{References}

D Lien, TJ (2003; 5). Cervical Spine Clearance in Australian Intensive Care Units. Critical Care and Resuscitation, 91-96. Seiler WO, SH (1979). Skin Oxygen tension as a function of imposed skin pressure: implication for decubitus ulcer formation. Journal for American Geriatric Society, 27:298-301.

\section{Polysomnographic characteristics of submental surface EMG} in sleep apnoeic patients

Mak Daulatzai ${ }^{1}$, Chandan Karmakar ${ }^{1}$, Ahsan Khandoker ${ }^{1}$, Neela Khan ${ }^{1}$, Marimuthu Palaniswamy ${ }^{2}$

${ }^{1}$ University of Melbourne, ${ }^{2}$ Swinburne University

Obstructive sleep apnoea (OSA) is characterised by periodic hypoxia/ reoxygenation owing to repetitive collapse of the upper airway during sleep. It is a serious disorder with increased risk of various comorbid conditions. Polysomnogram (PSG) is the current gold standard in the assessment of OSA. In addition to signals from brain, heart, eye, nose, and thoraco-abdominal sites, it also records surface submental electromyography (sSM EMG) signals. We have evaluated PSGs from 65 OSA patients (25-70 years) and analysed over ten thousand epochs and one thousand hypopneas in patients with varying apnoeahyperpnoea index (AHI). In low and moderate AHI, the sSM EMG activity in hypopnoeas varies both qualitatively and quantitatively as either linearly high, linearly low, or 'chimeric'. However, in patients with an AHI of 85+, the SSM EMG pattern displayed unique phenotypic activity characteristics in their hypopnoeas. These were divisible into three distinct categories as (1) Showing gradually escalating higher activity culminating first in a fusiform or globular pattern, and then in thick coarse varicosities of activity, (2) A reverse of the 1, i.e. first a much higher activity tapering to low, and (3) The higher activity present only in the middle segment of the hypopnoea, simulating an inverted $\mathrm{V}$ configuration. We have shown that $\mathrm{SSM}$ EMG is a dynamic and highly reactive myoneural system, being comprised of tonic, and episodic phasic contracting motor units of differing activity intensity at different AHI. In this paper, the present sSM EMG data are correlated with disease progression, and the attributes of innervating neurons.

Comparative analysis of computational flow dynamics in cerebral saccular aneurysms using steady and pulsatile flow

\section{Azadeh Farnoush $^{1}$, Yi Qian ${ }^{1}$, Albert Avolio ${ }^{1}$}

${ }^{1}$ The Australian School of Advanced Medicine, Macquarie University

Recent advances in numerical modelling software and microprocessor capacity have enabled application of computational techniques to assessment of haemodynamic factors related to rupture of cerebral aneurysms. However, computation time using finite element models of arterial structures depends on the specification of input flow, with a large difference in computation time between steady and pulsatile flow. The aim of this investigation was to compare relative differences in total flow with aneurysms of varying sizes and with different geometry of parent vessels.

Numerical simulations were performed on saccular aneurysms of 6,8 and $10 \mathrm{~mm}$ depth being supplied with either curved or straight parent vessels of 4,5 , and $6 \mathrm{~mm}$ diameter using commercial software (ANSYS). Calculations of intra-aneurysmal flow relative to parent vessel flow were performed using either a steady flow rate $(0.3 \mathrm{l} / \mathrm{min})$ or a physiological pulsatile flow with similar mean value and range $0.19-0.42 \mathrm{l} / \mathrm{min}$.

For a given aneurysm size, intra-aneurysmal flow was greater for curved compared to straight parent vessels with both pulsatile and 
steady input flow. Although aneurysmal flow for peak calculations are relatively greater, relative difference of aneurysmal flow for different aneurysm size when normalised for a $10 \mathrm{~mm}$ depth was similar for curved and straight vessels when using steady and pulsatile flow (steady: $0.0 \pm 2.0 \%$; pulsatile: $3.0 \pm 3.4 \%$ ). Computation time was 20 times greater for pulsatile flow.

For comparative computational fluid dynamics simulations of intra-aneurysmal flow, use of steady input flow produces minimal error compared to pulsatile flow, but has a significant reduction in computational time.

\section{Non-invasive estimation of cerebral critical closing pressure from transcranial doppler flow and peripheral and central pressure pulse}

\section{Mi Ok Kim ${ }^{1}$, Alberto Avolio ${ }^{1}$}

${ }^{1}$ Macquarie University

Background Cerebral blood pressure and flow are generally linearly related. However, they are not proportionally related due to the presence of a critical closing pressure (CCP) (i.e. the pressure at cessation of flow). Determination of CCP has been shown to be a reliable means of quantification of cerebral flow regulation.

Aim The aim of this study was to compare estimates of CCP using non-invasive transcranial doppler (TCD) flow and peripheral pressure pulse with estimates using TCD and central aortic pressure.

Methods TCD blood flow velocity (CBFV) in the middle cerebral artery and simultaneous radial artery pressure pulse (pABP) obtained by applanation tonometry were recorded in six healthy subjects (age 24-31 years, two females). Central aortic pressure (cABP) was estimated from pABP using the SphygmoCor device. CCP was calculated using the classical linear regression (LR) of the instantaneous CBFVpABP and CBFV-cABP (CCPp and CCPc, respectively). CCP was also estimated by LR of the first harmonic component of CBFV and the first harmonic of pABP and cABP (CCPp_H1 and CCPc_H1, respectively).

\section{Results}

\begin{tabular}{lllll}
\hline & CCPc_H1 & CCPp_H1 & CCPc & CCPp \\
\hline Mean & $29.7 \pm 8.1$ & $28.8 \pm 9.7$ & $30.3 \pm 11.2$ & $22.9 \pm 12.2^{*}$
\end{tabular}

Mean $\pm \mathrm{SD} ; * P<0.025$ in relation to all other $\mathrm{CCP}$ estimates

Conclusion This study has shown that non-invasive assessment of CCP using TCD and central aortic pressure produces a more consistent estimate of CCP than using TCD and the peripheral pressure pulse.

\section{Non-invasive detection of bilirubin in discrete vessels}

Mark McEwen ${ }^{1,2}$, Karen Reynolds ${ }^{1}$

${ }^{1}$ School of Informatics and Engineering, Flinders University, ${ }^{2}$ Flinders Biomedical Engineering, Flinders Medical Centre

Bilirubin, at the levels encountered in hyperbilirubinaemia, makes a similar contribution to the blue light absorption, as reduced haemoglobin makes to the red light absorption of blood. Whilst this supports the possibility of extending pulse oximetry into the blue part of the spectrum-for the non-invasive monitoring of bilirubin, the modulating effects of discrete blood vessels tend to disrupt the relationship between blood absorption coefficients and pulsations in light transmission through tissue.
The relationship between the pulsatile attenuation of blue and green light, and serum bilirubin concentration was investigated with a Beer-Lambert pulse oximetry model, a discrete blood vessel model and in vivo. Whilst the Beer-Lambert model indicated a strong positive relationship between the blue/green light pulsatile attenuation ratio and serum bilirubin, both in vivo testing and the discrete blood vessel model demonstrated a negative relationshipwhich was too weak to be used for bilirubin estimation.

Air guard decreases the prevalence of air/oxygen misconnections

Mark McEwen ${ }^{1,2}$, Glen Kennet ${ }^{2}$, Anne-Louise Smith ${ }^{1,2}$, Robin Woolford ${ }^{2}$

${ }^{1}$ School of Informatics and Engineering, Flinders University,

${ }^{2}$ Flinders Biomedical Engineering, Flinders Medical Centre

Connecting the wrong gas to a patient who needs oxygen, is a serious and sometimes tragic event. In 2006 there was an increase in the number of air/oxygen misconnection incidents reported to AIMS (Australian Incident Management System), at Flinders Medical Centre (FMC). As a result, FMC introduced the 'Air Guard'. The Air Guard is fitted to the outlet of an air flow meter, and requires clinical staff to move it, before a hose can be connected to the flow meter. The effectiveness of the Air Guard, at reducing the prevalence of air/ oxygen misconnection instances has been monitored at Flinders Medical Centre since its introduction in 2007. During that time, the number of incidences reported per annum has decreased by over $50 \%$ when compared to the previous 2 years.

Preliminary validation of an artifact detection algorithm designed for use within a new depth of anaesthesia monitor

Stacey Pritchett ${ }^{1,2}$, Eugene Zilberg ${ }^{2}$, Zheng Ming $\mathrm{Xu}^{2}$, Ian Brown ${ }^{1}$, Paul Myles ${ }^{2,3}$, David Burton ${ }^{1,2}$

${ }^{1}$ Monash University, ${ }^{2}$ Compumedics Ltd, ${ }^{3}$ The Alfred Hospital

When automatically processing EEG for any application, it is well recognised that appropriate detection of artifacts is needed. An EEG artifact detection algorithm based on pattern recognition has been developed for use within a new depth of anaesthesia monitor and this study is designed to validate the artifact detection. EEG was recorded from 44 subjects undergoing general anaesthesia for day surgery. The EEG was divided into $10 \mathrm{~s}$ intervals and artifacts were allocated to belong to one of three categories: eye blinks, body movement and other artifacts (i.e. electrosurgery; large, sharp or sudden changes and other abnormal EEG patterns, excluding burst suppression). Each type of artifact was visually recorded as present if it occurred one or more times in each interval, and sections of EEG determined as artifact by the algorithm were recorded as present similarly. The sensitivity and specificity of the algorithm detecting eye blinks was 86.89 and $98.51 \%$ respectfully. Body movement detection results were $12.41 \%$ sensitivity and $99.68 \%$ specificity and the detection of other artifacts resulted in $86.80 \%$ sensitivity and $94.72 \%$ specificity. The overall results of sensitivity and specificity of any artifact being both present and detected; regardless of the artifact type was 81.21 and $95.92 \%$ respectfully. The conclusion of this analysis is that there is a high sensitivity and specificity of the overall artifact detection. Each type of artifact detected will be processed in a different manner in the depth of anaesthesia algorithm and as such it is important to follow up these preliminary results with a more in depth study of where the artifact detection may possibly be improved. 
A forward-error-correction-based scheme for transmitting high volume of biomedical data through wireless communication channels with low data rate

\section{Johnson Thie ${ }^{1}$ \\ ${ }^{1}$ Australian School of Advanced Medicine}

Wireless communication is a convenient means of transferring data, however its main drawback is low data rate. Increased data rate for wireless transmission requires much higher power consumption which disadvantages portable devices. We propose a method for transmitting high volume of biomedical data from a portable device through wireless communications with low data rate.

The system has been developed using NORDIC Semiconductor's wireless modules and tested on 16 channels of EEG sampled at $1 \mathrm{kHz}$ and digitised at 16 bits per sample. The wireless timing constraints and the amount of data make it impossible to re-transmit data when it is corrupted by noise. The system employs optimal allocation of forward-error-correction (FEC) codes to the data. Data bits are rearranged such that more significant bits can be immediately recovered when less significant bits are recoverable. The optimisation strategy employs a local search hill-climbing algorithm which exhibits faster convergence and hence suits on-the-fly processing.

Experimental results demonstrate that the method yields minimal data degradation until the probability of packet loss reaches 0.2. When data are first compressed using a lossless algorithm, the probability increases to 0.3 . In contrast, transmitting data once without FEC codes results in data degradation when the probability is at least 0.05 .

In conclusion, the proposed FEC-based scheme improves reliability of data transmission when there is no provision for retransmission. The improvement is further enhanced when data are compressed allowing addition of more FEC codes.

\section{An objective method for measurement of contact quality} of surface electrodes and for cancellation of EEG attenuation

\section{Johnson Thie ${ }^{1}$, Alberto Avolio ${ }^{1}$ \\ ${ }^{1}$ Australian School of Advanced Medicine}

In EEG recording, operators apply conductive gel while inspecting the EEG signal. This task requires skilled operators, hence EEG quality becomes a subjective measure. We propose an objective method to measure contact quality of surface electrodes and in turn cancel signal attenuation. The method involves application of $1 \mathrm{mV}$ $100 \mathrm{kHz}$ square-wave to the scalp through an additional electrode so that the EEG signal contains the square-wave.

The double time-constant skin-electrode model is used, which assumes identical skin-electrode conditions. Applying a narrow bandpass filter at $100 \mathrm{kHz}$ yields an attenuated square wave. Its ratio with the original square wave provides an index of contact quality. Its magnitude response is flat between $6 \mathrm{~Hz}$ and $100 \mathrm{kHz}$. For variation of resistor and capacitor of the skin model with fixed electrode model, the difference in the magnitude response between $6 \mathrm{~Hz}$ and $100 \mathrm{kHz}$ remains within $5 \%$ until the resistor and capacitor drop below $1 \mathrm{kohm}$ and $10 \mathrm{nF}$ respectively. The EEG is scaled by this index to cancel attenuation from skin-electrode interface. For identical skinelectrode interface, the scaling error is at most $5 \%$ if the respective difference in the resistors and capacitor values for each interface is within three orders of magnitude.

This study has shown that applying a square wave enables objective measurement of contact quality of electrodes. Attenuation of the square-wave can be used to scale the EEG magnitude to cancel the attenuation in the EEG signal.
An application of ICA for novel methodology of normogastric rhythm extraction from electrogastrographic signal

\author{
Ewaryst J Tkacz ${ }^{1}$, Pawel S Kostka ${ }^{1}$ \\ ${ }^{1}$ Silesian University of Technology, Institute of Electronics, Division \\ of Microelectronics and Biotechnology, Poland
}

Electrogastrographic signal (EGG) is considered to be one of the less interesting from both registration and interpretation point of view. There are several reasons of that two facts. EGG presents gastric myoelectrical activity measured by several electrodes attached on the abdomen.
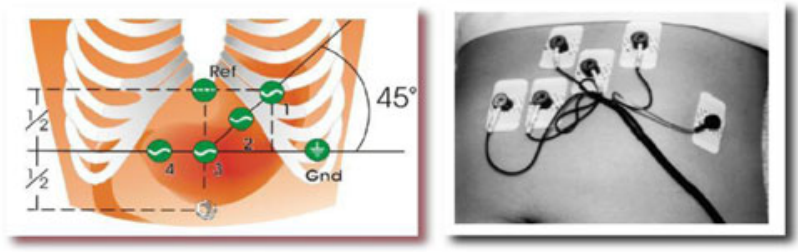

Fig. 1 Electrode placement for 4-channel EGG signal registration

Unfortunately the registration procedure does not deliver a pure signal as EGG is usually associated with some inferences caused by the other organs localized near stomach. On the other hand however there are no databases available, which could allow both comparison and proper interpretation. One of the parameter, among others, which is analysed owing to proper registration is so called normogastric rhythm, which should cover around $70 \%$ of rhythmic behaviour of the signal. Proper extraction of the normogastric rhythm is a subject of this paper. Special signal preprocessing steps should be applied before the main tool i.e. Independent Component Analysis (ICA) is applied for normogastric rhythm extraction. Also, to make this analysis possible a special registration procedure has been applied concerning two phases of registration-one with feeding and the other one without with $5 \mathrm{~min}$ brake between them.

Generally, there are two types of muscle electrical activity inside the stomach: Electrical Control Activity (ECA) or so called slow wave and Electrical Response Activity (ERA). Slow waves are spread out from one cell to another of longitudinal layer owing to electrotonic currents in the circular layer. Slow waves frequency is around 3 cycles per minute $([\mathrm{cpm}])$ and do not produce contractions of the stomach muscles.

Estimation of arterial pressure from features of the peripheral pressure pulse by means of a multi-regression model

Ke Xu ${ }^{1}$, Mark Butlin ${ }^{1}$, Alberto Avolio ${ }^{1}$

${ }^{1}$ Macquarie University

Changes in arterial pressure are associated with changes in shape of the arterial pulse. This association can be used for continuous noninvasive estimation of arterial pressure by analysis of waveform features. The aim of this study was to assess use of harmonic analysis of the peripheral radial pressure pulse for estimation of systolic (SBP) and diastolic pressure (DBP).

Brachial cuff pressure was measured in 10 supine subjects (age 21-34 years, heart rate 51-85 bpm) and the radial pulse was obtained in the same arm using applanation tonometry. Harmonic components were obtained by Fast Fourier Transform. Multiple linear regression models were constructed using heart rate, age and harmonics (1st to 5th) as predictor variables for SBP and DBP. Models were constructed in nine subjects and the other used as a test subject. This was 
done for all combinations of training and test subjects and predicted values compared with measured values for the whole group.

For the ten iterations, estimated values were SBP $(111 \pm 9)$, DBP $(70 \pm 9)$ compared to measured values: SBP $(111 \pm 7)$ and DPB $(69 \pm 4)$. Mean differences and standard deviation $(\mathrm{SD})$ were 0 and $3.3 \mathrm{mmHg}$ for SBP and 1 and $7.7 \mathrm{mmHg}$ for DBP respectively. The estimated pressure was within the criterion of Association for the Advancement of Medical Instrumentation which specifies a mean difference of $5 \mathrm{~mm} \mathrm{Hg}$ and SD of $8 \mathrm{mmHg}$.

This study has shown that SBP and DBP can be reliably estimated in supine young healthy subjects using features of the peripheral radial pressure pulse.

\section{Medical physics}

Cytokinesis-block micronucleus assay indicates long-term reproducibility of blood toxicity

\section{Obaidur Rahman ${ }^{1}$, Emma Y Song ${ }^{2}$, Barry J Allen ${ }^{1}$}

${ }^{1}$ Centre for Experimental Radiation Oncology, Cancer Care Centre, St George Hospital and St George Clinical School, University of NSW, ${ }^{2}$ Cord and Marrow Transplant Facility, Sydney Children's Hospital

Radiation dosimetry measures the absorbed dose from ionising radiation. Biological dosimetry includes the biological effects arising from structural chromosomal aberrations. The cytokinesis-block micronucleus assay allows the micronuclei (MN) to be counted, providing an in situ biological dosimeter. Four years ago Song et al. (2005) evaluated the MN frequency in peripheral blood lymphocytes after in vitro incubation with the alpha conjugates (AIC), ${ }^{213} \mathrm{BiI}_{3}$ and ${ }^{213} \mathrm{Bi}-9.2 .27$. Five patients were injected with $260-360 \mathrm{MBq}$ of AIC, and blood samples were taken before treatment; $3 \mathrm{~h}, 2$ and 4 weeks post treatment. Microscopic slides were prepared and the MN frequency was determined by the analysis of 1000 binucleated cells per subject under the light microscope with a magnification of $1000 \times$ (Fenech 1993). Four years later the microscopic slides were again observed for scoring the micronuclei frequency by using Axio Cam, a computer accessible microscope, for keeping the image in digital form to regenerate the data in future. It is found that there is no significant difference in the counts over the time. The cytokinesisblock micronucleus assay was shown to store the radiation-induced chromosome damage in lymphoma cell lines in terms of micronuclei frequency over 4 years.

\section{References}

Fenech M 1993, The cytokinesis-block micronucleus technique: a detailed description of the method and its application to genotoxicity studies in human populations. Mutat. Res. 285: 35-44.

Song et al 2008, The cytokinesis-block micronucleus assay as a biological dosimeter for targeted alpha therapy. Phy. Med. Bio., 53: 319-28.

\section{Computerised four-electrode focused impedance measurement system for physiological study}

\section{Obaidur Rahman ${ }^{1}$, KS Rabbani ${ }^{2}$, Barry J Allen ${ }^{1}$}

${ }^{1}$ Centre for Experimental Radiation Oncology, Cancer Care Centre, St George Hospital and St George Clinical School, University of NSW, ${ }^{2}$ Department of Biomedical Physics and Technology, University of Dhaka, Bangladesh
Focused impedance measurement (FIM) is a new electrical impedance technique conceived and developed by the Biomedical Physics Group of Dhaka University to localise a zone of interest. It may be applied for physiological study and diagnosis of large organs like lungs, stomach and heart, both in the frontal plane and in the transverse plane of the human thorax. There is also potential for its use in the identification and localisation of certain tumours, for monitoring temperature during radiotherapy, and for monitoring of the healing. Two versions of FIM were developed, one uses six electrodes while the other uses four electrodes. In the latter the four electrodes are located at the corners of a square matrix. Current is driven through an adjacent electrode pair while the potential is measured across the opposite pair giving an impedance value (say $\mathrm{Z}_{1}$ ). Similar impedance values (say $\mathrm{Z}_{2}, \mathrm{Z}_{3}$ and $\mathrm{Z}_{4}$ ) are obtained by rotating the connections to the electrode assembly in sequence. The sum of these four impedance values has a dominant contribution from the central region within the square matrix, giving the desired focusing. The present work was taken up to develop a PC based four electrode FIM system. A constant current generator and a differential potential measuring circuitry were developed using op-amps and the electrodes were switched to these two circuits through analogue switches. Control outputs from the parallel port of the PC controlled the analog switches while the analogue output of the potential measuring circuitry, which is in the form of a dc voltage, is acquired using an analogue interface having an 8 bit A/D converter, also developed in the present work. Developing suitable software will allow this computerised four electrode FIM system to be applied for physiological study and diagnosis.

Synthesis of evoked nerve action potential with and without consideration of circulating currents around a depolarised zone

\section{Obaidur Rahman ${ }^{1}$, KS Rabbani ${ }^{2}$, Barry J Allen ${ }^{1}$}

${ }^{1}$ Centre for Experimental Radiation Oncology, Cancer Care Centre, St George Hospital and St George Clinical School, University of NSW, ${ }^{2}$ Department of Biomedical Physics and Technology, University of Dhaka, Bangladesh

A nerve trunk consists of thousands of nerve fibres with different signal conduction velocities. Normal persons have a typical pattern of distribution of conduction velocity (DCV) of the individual nerve fibres while it differs in cases of disease or disorder. An ideal diagnosis would require detailed information on DCV but no technique has yet been developed to extract DCV of sensory nerve fibres from experimental measurements. An evoked nerve conduction study basically gives the compound nerve action potential (CNAP), which is a space and time dependent combination of all the individual single fibre action potentials (SFAP) recorded from a nerve trunk under test, on artificial stimulation at one or more suitable points. In order to relate the patterns of measured CNAP to different DCV, synthesis of CNAP's were carried out in the present work for different assumed DCV patterns using a simple model for the SFAP. Two methods were used for this work, in one, the time spread of an action potential was taken to equal the time of travel of a depolarised zone in the nerve fibre under a measuring electrode placed some distance away on the skin. All reported similar work used this assumption. In the other method the contribution of circulating currents that flow near the two edges of the depolarised zone were considered which result in an increased time spread of the measured action potential. This simulation work increased our understanding of the CNAP patterns, which may be useful in extracting qualitative information on DCV variations leading to a better diagnosis. 
Monte Carlo modelling of combined dose distributions in breast radiotherapy

\section{S Bensaleh ${ }^{1,2,3}$, E Bezak $^{2,3}$}

${ }^{1}$ Renewable Energy Research Centre, Libya, ${ }^{2}$ Department of Medical Physics, Royal Adelaide Hospital, ${ }^{3}$ Schools of Chemistry and Physics, University of Adelaide

Introduction At our institution, patients with early stage breast cancer were treated with the combination of external beam radiotherapy (EBRT) and high dose rate (HDR) MammoSite brachytherapy (MB). The treatment consisted of 50 Gy dose delivered in 25 fractions using wedged tangential photon beams (6 MV), followed by a boost of 9-10 Gy to the tumour bed delivered using the MammoSite applicator. It would be highly beneficial to visualise the final (combined) dose distribution from the two modalities to assist with treatment planing and with the assessment of treatment outcomes. In this study, the EGSnrc Monte Carlo (MC) code is used to calculate the dose distributions using computed tomography $(\mathrm{CT})$ images of a patient treated with the combination of MB and EBRT.

Methods In this work the brachytherapy source (MicroSelectron HDR ${ }^{192}$ Ir) was modelled as a parallelepiped of rectilinear coordinates of $0.6 \times 0.6 \times 3.6 \mathrm{~mm}^{3}$ using DOSXYZnrc ${ }^{1}$ code. The photon emission from the modelled ${ }^{192} \mathrm{Ir}$ source was assumed to be isotropic. The $\gamma$-ray spectrum for the ${ }^{192} \mathrm{Ir}$ was taken from a published report and consisted of 34 energy bins ranging from 0.060 to $0.885 \mathrm{MeV}$ [2]. The BEAMnrc ${ }^{3} \mathrm{MC}$ code was used to simulate the Varian $600 \mathrm{C} / \mathrm{D}$ (Varian Oncology Systems, CA) linac with 6 MV X-ray beam. The model of the Linac was previously generated and has been expanded for wedged fields in the current work [4]. Simulations were performed using physical wedges of angles $15,30,45$ and $60^{\circ}$ and $10 \times 10 \mathrm{~cm}^{2}$ field size. DOSXYZnrc code was also used to calculate the dose distribution using the patient $\mathrm{CT}$ data treated with the two techniques. Combining the 3D dose distribution from the two modalities is not straightforward due to differences in delivery mode, fraction size, dose rate and energy associated with each modality. A MATLAB program has been developed to extract the $3 \mathrm{D}$ dose distribution matrix from MC simulations. The concepts of equivalent dose and biological effective dose were used to combine the 3D dose distribution from EBRT and brachytherapy.

Results and discussion The results of MB modelling were verified with TLD measurements and were in a good agreement (within 2\%). Simulations for open and wedged fields showed agreement with measured profiles and depth doses to within $2 \%$.

Conclusions The MC models have been verified with measurements. The results indicate that accurate models have been constructed. In the next step, the physical dose will be converted into biological effective dose and the dose distributions from the two modalities will be combined.

\section{References}

1. Walters B, Kawrakow I, Rogers D. (2005) DOSXYZnrc user's manual, Techn. Rep. No. PIRS-794, RevB. National Research Council of Canada, Ottawa, Canada.

2. Borg J, Rogers D. (1999) Spectra and air-kerma strength for encapsulated 192Ir sources. Med Phys 26:2441-4.

3. Rogers D, Faddegon B, Ding G et al. (1995) BEAM: a Monte Carlo code to simulate radiotherapy treatment units. Med Phys 22:503-24.

4. Thuc P. (2009) Simulation of the transmitted dose in EPID using a Monte Carlo method. M.Sc. thesis, University of Adelaide.

\section{Dose response of the highest sensitivity radiochromic film dosimeters available}

Martin J Butson ${ }^{1,2,3}$, Tsang Cheung ${ }^{1}$, Peter $\mathrm{KN} \mathrm{Yu}^{1}$, Hani Alnawaf ${ }^{3}$

${ }^{1}$ Department of Physics and Materials Science, City University of Hong Kong, ${ }^{2}$ Department of Medical Physics, Illawarra Cancer Care
Centre, ${ }^{3}$ Centre for Medical Radiation Physics, University of Wollongong

The spectral absorption properties in the visible wavelengths have been investigated for XR-QA radiochromic film and results show two main peaks in absorption located at 636 and $585 \mathrm{~nm}$. A much higher sensitivity however is found at kilovoltage energies with an average $1.55 \mathrm{OD}$ units per $20 \mathrm{cGy}$ irradiation variation measured at $636 \mathrm{~nm}$ using $150 \mathrm{kVp} \mathrm{X}$-rays. This is compared to approximately $0.12 \mathrm{OD}$ units per $20 \mathrm{cGy}$ measured at $636 \mathrm{~nm}$ for EBT film at $6 \mathrm{MV}$ X-ray energy. That is, the XRQA film is more than ten times more sensitive than EBT film. The visual colour change is enhanced by the yellow polyester coating however this does not effect the absorption spectra properties in the red region of analysis which is the main area for use using desktop scanners in reflection mode. The film is suited for low dose applications using kilovoltage X-rays which may include CT dose assessment and in-vivo dosimetry for radiotherapy kilovoltage treatments.

Effects of radiation scatter exposure on electrometer dose assessment in superficial radiotherapy

Martin J Butson ${ }^{1,2,3}$, Tsang Cheung ${ }^{1}$, Peter $\mathrm{KN} \mathrm{Yu}{ }^{1}$

${ }^{1}$ Department of Physics and Materials Science, City University of Hong Kong, ${ }^{2}$ Department of Medical Physics, Illawarra Cancer Care Centre, ${ }^{3}$ Centre for Medical Radiation Physics, University of Wollongong

During superficial and orthovoltage X-ray radiotherapy dosimetry normal practice requires the use of a standard ionisation chamber and dedicated electrometer for dosimetry. In ideal conditions, the electrometer is positioned outside the treatment room to eliminate any effects from scatter radiation on dose measurement. However in some older designed rooms, there is no access portal for the chamber cable to run to an 'outside' position for the electrometer. As such the electrometer is positioned within the treatment room. This work quantifies the effects on measured charge when this occurs. Results have shown that with the electrometer positions next to the solid water dosimetry stack for a larger $15 \mathrm{~cm} \times 15 \mathrm{~cm}, 250 \mathrm{kVp}$ beam, charge results can deviate by up to $\pm 7.5 \%$ depending on the polarity applied to the chamber compared to readings when the electrometer is outside the treatment room. These values follow the same pattern but differ by quantity for different designed electrometers such as a NE 2571 and PTW unidose. It is assumed to be due to the causing material construction of the two devices providing different absorption and scattering conditions within the electrometers amplifying circuits. An qualitative exposure relationship is defined and shown for the NE electrometer. Results are also shown when the electrometer is shielded by lead whilst inside the room. Whilst it is well known that an electrometer should not be irradiated, often small kilovoltage or orthovoltage rooms do not have a portal access for an electrometer to go outside. As such it would be recommended for a lead shield to be placed around the electrometer during irradiation if this was to occur to remove effects of scatter radiation.

\section{Measuring solar ultraviolet radiation with EBT radiochromic film} Ethan Butson ${ }^{1}$, Margaret Dyos ${ }^{1}$, Martin Butson ${ }^{2}$

${ }^{1}$ The Illawarra Grammar School, ${ }^{2}$ Department of Physics and Materials Science, City University of Hong Kong

EBT Gafchromic film is designed for use with X-ray radiation in applications such as radiotherapy cancer treatment. The film changes from a clear to a blue colour automatically when exposed. The film 
however has also been observed to change colour slowly whilst exposed to ultraviolet radiation from the sun. As such, a study has been performed to evaluate the ability of the radiochromic film to measure solar ultraviolet radiation quantitatively. Results have shown that the film produces a colour change which is measurable and can be fitted by a polynomial function to measure ultraviolet radiation. The film results were calibrated against a broad spectrum UV dosimeter. Results showed that up to $15 \%$ variation in optical density to exposure level was measured using the EBT film when the solar radiation source varied from sunny summer day to cloudy winter days. Variations up to $5 \%$ were measured during a normal day from morning through to evening exposure. As such the film provides a simple yet effective measure of ultraviolet radiation exposure to broadband UV rays. As the film can be used as a two-dimensional dosimetry the film can measure radiation exposure on areas such as around eyes, behind sunglasses and other areas where a high level of spatial; resolution may be needed.

\section{Methods to improve MOSFET dosimetry at kilovoltage energies}

Martin J Butson ${ }^{1,2}$, Tsang Cheung ${ }^{1}$, Peter $\mathrm{KN} \mathrm{Yu}^{1}$

${ }^{1}$ Department of Physics and Materials Science, City University of Hong Kong, ${ }^{2}$ Department of Medical Physics, Illawarra Cancer Care Centre

All radiation dosimeters exhibit some form of energy dependence depending on their materials of construction and design specifications. Various commercial MOSFET devices are known to have different energy dependences especially in the kilovoltage range as the active material is predominantly made from silicon and the construction materials and housing can include other high density materials such as metal wires. This work looks at the dose response of a new form of CSDS MOSFET device when irradiated with various kilovoltage X-ray energies and explains a method for correction to the dose response sensitivity over the life of the MOSFET dosimeter. Results have shown that for this device, a function can be applied to dose sensitivity with age which is dependant on beam energy and as such corrections can be made effectively at one energy of irradiation as results translated to another beam energy. This would aid in increasing the life of the MOSFET dosimeter.

\section{Remember which way is up! Polarisation Effects on radiochromic} films

\section{Martin J Butson ${ }^{1,2,3}$, Tsang Cheung ${ }^{1}$, Peter $\mathrm{KN} \mathrm{Yu}{ }^{1}$}

${ }^{1}$ Department of Physics and Materials Science, City University of Hong Kong, ${ }^{2}$ Department of Medical Physics, Illawarra Cancer Care Centre, ${ }^{3}$ Centre for Medical Radiation Physics, University of Wollongong

It is well known that most radiation dosimetry film products such as radiographic and radiochromic films exhibit what is known as orientation effects. This is specifically due to the light polarising nature of the films construction materials and active layers. Manufacturers recommend that scanning occur in the same orientation (landscape or portrait) for calibration and experimental films. This work has utilised a $>99 \%$ plane polarised light source and a nonpolarised diffuse light source to investigate the absolute magnitude of EBT Gafchromic films polarisation or orientation effects. Results have shown that using a non-polarised light source produces a negligible orientation effect for EBT Gafchromic film and thus the angle of orientation is not important. However, the film exhibits a significant variation in transmitted optical density with angle of orientation to polarised light producing more than $100 \%$ increase, or over a doubling of measured OD for films. I.e. The degree of polarisation of the scanning light source controls the variation in the orientation effect. As each desktop scanner or densitometer will have different properties, this degree of polarisation should be quantified and measured for each device used. The position of the maxima optical density change for EBT film was found to be at approximately $14 \pm 7^{\circ}(2 \mathrm{SD})$ when off the films natural 'landscape' axis. This means that it is crucial to not only scan in the same orientation for all films but to also scan with the same 'top and bottom' film sides to remove the off axis polarisation effect.

Scanning orientation and polarisation effects for XRQA radiochromic film

Martin J Butson ${ }^{1,2,3}$, Hani Alnawaf ${ }^{3}$, Tsang Cheung ${ }^{1}$, Peter $\mathrm{KN} \mathrm{Yu}{ }^{1}$

${ }^{1}$ Department of Physics and Materials Science, City University of Hong Kong, ${ }^{2}$ Department of Medical Physics, Illawarra Cancer Care Centre, ${ }^{3}$ Centre for Medical Radiation Physics, University of Wollongong

Gafchromic XRQA radiochromic film, is an effective tool for quality assurance and dose assessment in kilovoltage radiotherapy and diagnostic applications. Like other Gafchromic film products, XRQA film exhibits a variation is dose to optical density response with angle of rotation when analysed with a light source that is partially or fully polarised such as a desktop scanner. Although warnings are not given on manufacturers specifications, this can affect dosimetry accuracy and we recommend that it is essential to scan all XRQA films in the same orientation. The effect is not as pronounced as EBT Gafchromic film. The magnitude of this variation has been measured and shown to be up to $17 \pm 2 \%$ ( $1 \mathrm{SD}$ ) using a fully linear polarised light source was seen with a $90^{\circ}$ angle rotation. For our standard desktop scanner (Epson v700) this variation was measured as $2 \pm 1 \%$ as compared to $8 \pm 2 \%$ for EBT Gafchromic. Although the manufacturers do not provide a warning on their product to maintain scanner to film orientation for this type of film, we recommend that to increase accuracy, this be performed on a regular basis. This is especially important if your desktop scanner has a high degree of polarisation of its light source.

Re-commissioning a cone stereotactic treatment planning system using measurements from ionisation chamber, radiochromic films and Monte Carlo simulation

KW Chan ${ }^{1}$, T Knittel ${ }^{1}$, SM Heng ${ }^{1}$, R Smee ${ }^{1}$

${ }^{1}$ Department of Radiation Oncology, Prince of Wales Hospital

Our centre recently underwent recommissioning of the Radionics XKnife RT4 treatment planning system (TPS) for circular cones. This system is dedicated to stereotactic radiotherapy. The data required for the TPS are: tissue maximum ratio (TMR) from the surface to a depth of $20 \mathrm{~cm}$, beam profile at $5 \mathrm{~cm}$ depth and dose per monitor unit (D/MU) at the reference depth, $1.5 \mathrm{~cm}$. These data have to be collected for all available circular cones, with 0.5 and $4.5 \mathrm{~cm}$ being the smallest and largest cone size respectively.

Three methods were used to obtain the beam data required: ionisation chamber (Scanditronix- Wellhofer CC04) in water phantom, Radiochromic film (Gafchromic EBT) in solid water phantom and 
Monte Carlo Simulation (MCS) using EGS4 for a homogeneous water phantom.

Our results showed that MCS agree with ionisation chamber measurements for both TMR and D/MU of large cones $( \pm 2 \%)$. For smaller cone size, due to the lack of electronic equilibrium, ionisation chamber is no longer suitable. Spot checks using Radiochromic film (Gafchromic EBT) at selected depths were done because of its superior resolution and near energy independence response. MCS results agree with Gafchromic film to within $\pm 3 \%$ for both TMR and D/MU. For all profile measurements, the results of MCS match Gafchromic film to within $\pm 3 \%$.

The outcome of the recommissioning showed good agreement with the old data, with only minor adjustments needed

\section{Tumour response modelling based on biological image data}

Gwi Cho $^{1,2}$, Zdenka Kuncic ${ }^{2}$, Lois Holloway ${ }^{2,3}$, Clive Baldock ${ }^{2}$

${ }^{1}$ Department of Radiation Oncology, Royal Prince Alfred Hospital, Sydney South West Area Health Service, ${ }^{2}$ Institute of Medical Physics, School of Physics, University of Sydney, ${ }^{3}$ Liverpool Cancer Therapy Centre, Sydney South West Area Health Service

Introduction In radiation oncology, patient specific functional image data may be used to diagnose, stage, develop the treatment strategy and monitor the response of tumour to treatment. A widely utilised functional imaging modality is PET, where biological information, such as tumour hypoxia, proliferation and glucose metabolism are imaged in-vivo using ${ }^{18} \mathrm{~F}$-based tracers. While suitable metrics to utilise such biological information in radiotherapy treatments have not been fully identified, studies have been undertaken to use them quantitatively, such as in biologically optimised IMRT and in biologically adaptive therapy. In this work, an analytical approach is explored to quantify tumour response based on biological information using a Poisson TCP model.

Methods MATLAB ${ }^{\circledR}$ code was written using a DICOM image template to generate a voxelised tumour model (Fig. 1). A Poisson TCP model with its cell survival described by the LQ model was used to calculate TCP for clinically relevant treatments. Variation of TCP with initial clonogenic cell density up to $10^{9}$ cells per $\mathrm{cm}^{3}$ and $\alpha$ and $\beta$ linear quadratic parameters were evaluated.

Results and conclusion For the given model tumour, with constant $\alpha / \beta=10$, the TCP curve in Fig. 2 approaches a zero TCP at $\alpha=0.1$ and approximates a full TCP at $\alpha=8.4$. Between $\alpha=0.1$ and 8.4 the TCP curve is sigmoidal with its slope gradually increasing with higher $\alpha$ and $\beta$ parameters.

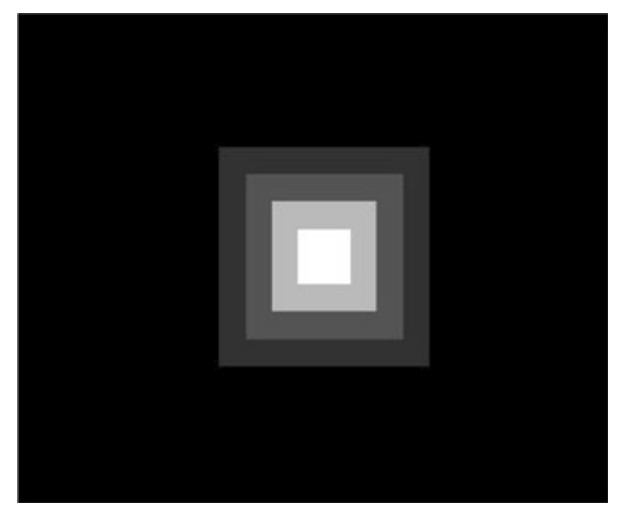

Fig. 1 Model tumour

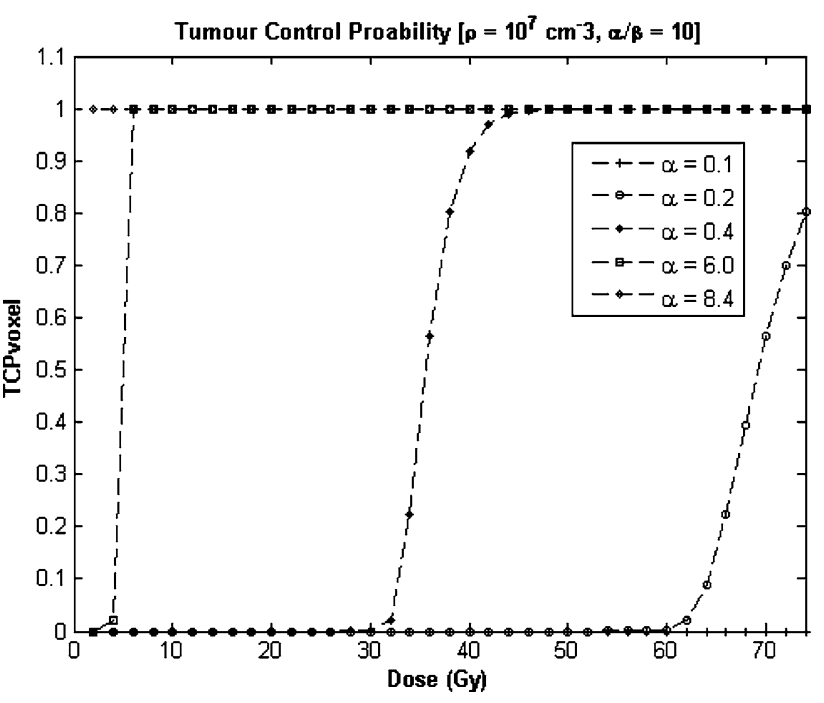

Fig. 2 TCP curves

Dosimetric effect of couch angle positional inaccuracy during conventional head and neck radiotherapy with non-coplanar beams

Thuc Pham ${ }^{1}$, Jacek Chojnowski ${ }^{2}$

${ }^{1}$ Nepean Cancer Care Centre, ${ }^{2}$ Westmead Cancer Care Centre

Introduction Non-coplanar beams are commonly used in conventional radiotherapy of head and neck $(\mathrm{H} \& \mathrm{~N})$ cancers. These beams are generated by moving the linac couch at an angle. Due to this technique's dependence on couch angle positional accuracy, it is essential to verify and ensure the correctness of this couch parameter. This study aims to describe and quantify the effects of couch angle errors during treatment with non-coplanar beams. It further presents the practical QA checks in ensuring correctness of couch angle position.

Method Inaccuracies of couch angle position during treatment were simulated on a conventional H\&N plan using Pinnacle TPS. Point doses, isodose distributions and DVHs of plans with 'introduced' couch angle errors were compared with a plan without such position discrepancies. The shift in the location of maximum dose was also collected in all treatment plans. Fluence maps for all plans were obtained, analysed and compared using OMNIPRO software. Couch positional accuracy was verified on Varian 21EX linac. Couch rotational axis was checked using the 'spoke shot' method. The couch $0^{\circ}$ position was also determined and the uniformity of the dose distributions of two orthogonal couch angle positions was checked using film.

Results Couch angle discrepancy of $\leq 3^{\circ}$ did not significantly alter the isodose distribution. The maximum dose changed by $0.3 \%$ and still located within the PTV. The PTV histogram remained the same. However, a shift of $3^{\circ}$ in couch position exceeded the optic chiasm tolerance dose by $89.5 \mathrm{cGy}$. The optic nerves also received a higher maximum dose but still within the dose limits. The couch $0^{\circ}$ position agreed within $0.2^{\circ}$ with the mechanical and digital scales. The 'spoke shot' showed a couch rotational axis within $1 \mathrm{~mm}$ radius. The junction between orthogonal couch angles was displaced by $1.5 \mathrm{~mm}$.

Conclusion Non-coplanar treatment technique is safe to use in radiotherapy when the couch angle accuracy is checked routinely and within tolerance. The isodose distribution, histogram and maximum 
dose location in the treatment plans used in this study did not change significantly unless the couch angle was incorrect by $\geq 3^{\circ}$.

\section{Development of real time in vivo rectal wall dosimetry with MOSFET detectors}

Nicholas Hardcastle ${ }^{1}$, Dean Cutajar ${ }^{1}$, Peter Metcalfe ${ }^{1}$, Michael Lerch $^{1}$, Wolfgang Tomé ${ }^{2}$, Anatoly Rosenfeld ${ }^{1}$

${ }^{1}$ Centre for Medical Radiation Physics, University of Wollongong, ${ }^{2}$ Department of Human Oncology and Medical Physics, University of Wisconsin, USA

Purpose To investigate real time in vivo rectal wall dosimetry with a novel clinical dosimetry system utilising a MOSFET based dosimeter, the MOSkin ${ }^{\mathrm{TM}}$.

Methods and materials A novel MOSFET detector, the MOSkin ${ }^{\mathrm{TM}}$, developed at CMRP was coupled to a commercial rectal balloon (RadiaDyne, Houston, TX, USA). The MOSkin ${ }^{\mathrm{TM}}$-Balloon apparatus was placed in a specifically designed phantom. A hypothetical prostate helical tomotherapy plan was delivered to the phantom and the rectal wall dose was measured in real time with the MOSkin ${ }^{\mathrm{TM}}$. The MOSkin ${ }^{\mathrm{TM}}$ measurements were compared with radiochromic film measurements and the TomoTherapy RTPS calculation. A correction method to account for MOSkin ${ }^{\mathrm{TM}}$ angular anisotropy was developed and applied to the dosimetry system.

Results The MOSkin ${ }^{\mathrm{TM}}$ measured dose was up to $7 \%$ lower than the radiochromic film measurement and up to $13 \%$ lower than the TomoTherapy Hi-Art Treatment Planning System (TPS) calculation for the tomotherapy delivery. The lower measured dose was due to a combination of an over prediction by the TPS and angular anisotropy of the MOSkin ${ }^{\mathrm{TM}}$. The angular anisotropy was corrected in a modified MOSkin ${ }^{\mathrm{TM}}$ detector. Excellent real time readout was obtained with the MOSkin ${ }^{\mathrm{TM}}$.

Conclusion The MOSkin ${ }^{\mathrm{TM}}$ dosimetry system, coupled with a rectal balloon, provides real time in-vivo dosimetry of the rectal wall, whilst immobilising the prostate during radiotherapy. The development of a correction method to account for angular anisotropy using a modified MOSkin design has shown promising results.

\section{Validation of plan-based calibration for relative dosimetry of intensity-modulated radiotherapy using an electronic portal-imaging device}

Shrikant Deshpande ${ }^{1}$, Philip Vial ${ }^{1,2}$, Gary Goozee ${ }^{1}$, Lois Holloway ${ }^{1,2,3}$

${ }^{1}$ Department of Medical Physics, Liverpool and Macarthur Cancer Therapy Centre, ${ }^{2}$ Institute of Medical Physics, University of Sydney, ${ }^{3}$ Centre for Medical Radiation Physics, University of Wollongong

Purpose To validate a plan-based calibration method implemented on a commercially available software program for relative dosimetry of intensity modulated radiation therapy (IMRT).

Methods and materials Radiological Imaging Technology image analysis software (RIT) contains plan based Calibration. An uncalibrated electronic portal-imaging device (EPID) image and a dose plane from a treatment planning system (TPS) were used. After manual registration, the RIT software maps the TPS dose to each EPID image pixel value and constructs a calibration curve. This calibration curve is then applied throughout to convert all EPID pixels to dose. The calibration curves from several different IMRT fields were compared to assess reproducibility. The calibrated EPID results were also benchmarked against measurements using a calibrated ionisation chamber array. The sensitivity of the plan based calibration method to delivery errors was also assessed. Gamma analysis with $3 \% / 3 \mathrm{~mm}$ tolerances were used.

Results The plan based calibration had ( 0.30 and 0.03$) \%$ of pixels Gamma $>1$ for two IMRT fields as opposed to MatriXX measurement (0.54 and 0.18$) \%$ respectively.

The calibration curves agreed to within $0.5 \%$ for both fields. The plan-based method was sensitive to registration errors but further work is required to determine its sensitivity to relative dose errors Conclusion The plan based calibration method appears to provide accurate dosimetry results. Further analysis is being conducted to better understand the limitations of this method.

Characterisation of thermoluminescence dosimetry energy response for use in high-dose-rate brachytherapy

\section{A Nguyen $^{1}$, A Haworth ${ }^{1,2}$, R Franich ${ }^{1}$ \\ ${ }^{1}$ School of Applied Sciences, RMIT University, ${ }^{2}$ Department of Physical Sciences, Peter MacCallum Cancer Centre}

Background and purpose Thermoluminescent detectors (TLDs) used for in-vivo dosimetry in $\operatorname{Ir}^{192}$ brachytherapy procedures are typically calibrated using photons from a medical linac. TLDs exhibit an energy dependence therefore a correction factor must be applied for brachytherapy applications. To determine the energy correction factor, the response of the TLDs was measured over a range of energies.

Methods LiF; Mg, Ti TLDs (TLD-100, Harshaw) were irradiated to a dose of $1 \mathrm{~Gy}$ in photon energies ranging from $10 \mathrm{keV}$ to $18 \mathrm{MV}$. The TLDs were also irradiated under a range of conditions (distance and media) using an HDR source. The energy response was determined using the curve fit of Kron et al. (Med Phys Biol 1998; 43: 32353259)

Results and discussion The response of the TLDs, normalised to the response at $6 \mathrm{MV}$ is shown in Fig. 1. Assuming a mean energy of $380 \mathrm{keV}$ for $\mathrm{Ir}^{192}$, the energy correction factor was 1.09 when compared directly with the measurements made in $6 \mathrm{MV}$ and 1.07 when interpolated from the response curve. When irradiated with $\operatorname{Ir}^{192}$ the response varied by $\pm 4 \%$ depending on the method of irradiation. Uncertainties in the $\operatorname{Ir}^{192}$ energy spectrum under varying irradiation conditions will be the subject of further investigations using Monte Carlo modelling.

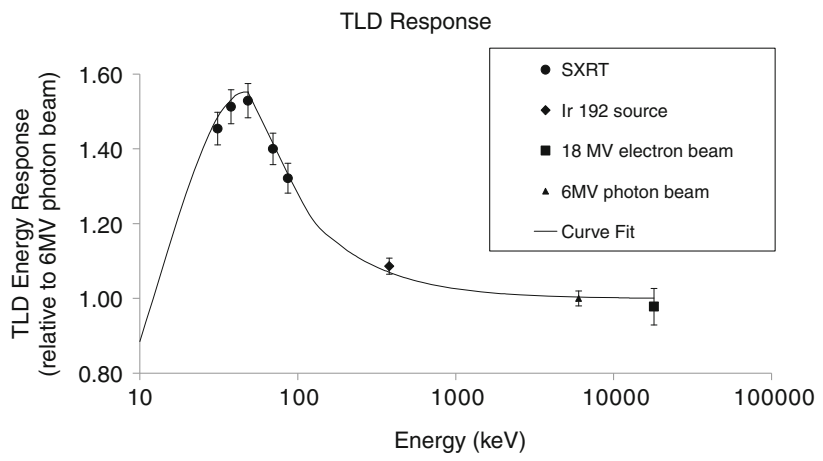

Fig. 1

Conclusions To reduce the uncertainties in the energy correction factor when TLDs have been calibrated for use with brachytherapy applications, it is recommended that the correction factor be determined using more than one method and preferably under conditions close to the in-vivo measurement conditions (energy and media). 


\section{Phenomenological model of radiobiology}

Sean Geoghegan ${ }^{1,3}$, Tegan Rourke ${ }^{2}$

${ }^{1}$ ACT Health, ${ }^{2}$ School of Physics, University of Western Australia, ${ }^{3}$ Royal Perth Hospital

The objective of radiotherapy is to deliver an optimal dose that maximises the eradication of a tumour while keeping the damage to the surrounding normal tissues within acceptable levels. To help achieve this, models have been developed to predict how tumours and normal tissues respond to radiation. A phenomenological model relates empirical observations in a way that is not directly derived from theory, whereas mechanistic models are derived from fundamental or underlying causes, however, mechanistic parameters can be very difficult to measure independently and/or reliably.

We investigated four mathematically comparable phenomenological models of survival (which we labelled RI, RII, RIII, RIV) that were based on derivative functions of a set of four easily determined and easy-to-control variables: total dose $D$, fractional dose $d$, total dose rate $\dot{D}$ and fractional dose rate $\dot{d}$. The four variables can be used as coordinates in a treatment domain. We show that displaying a treatment or model using the same four variables allows different treatment regimes to be compared more easily. We propose that derivative functions based on these four variables $(D, d, \dot{D}$ and $\dot{d})$ define parameters $(\chi, \Lambda, \Omega$ and $\gamma)$ that can characterise radiobiological survival models. The four standard radiobiological survival equations (the simple linear quadratic equation, the fractionated linear quadratic equation, the fractionated linear quadratic with repair equation and the fractionated linear quadratic with proliferation equation) can be accurately modelled from these derivative functions. If linearity is used as a test for appropriateness then the representation $\ln S(D, d, \dot{D}, \dot{d})$ is the most linear in the majority of cases and is also usually the most tolerant to noisy data (measured or calculated).

\section{Trends in Australian CT scan statistics 1994-2008}

Anna Hayton ${ }^{1}$, Anthony Wallace, Keith Edmonds, David Tingey ${ }^{1}$ ARPANSA

Purpose To examine the trends in CT use in Australia over the last 15 years. To compare the number of procedures performed per capita with other Health Care Level I Countries ${ }^{1}$. To compare the dose delivered to the population as a result of CT procedures with that of other Health Care Level I Countries.

Methods Medicare Australia ${ }^{2}$ provides comprehensive statics detailing (i) the total number of each item number claimed, (ii) the state in which the claim was made and (iii) the age and gender of the claimant. Descriptions of these item numbers provide a basis for grouping the individual item numbers according to the European Dose Datamed ${ }^{3}$ categories. The number of procedures performed in each category can then be multiplied by an average dose to give an overall dose to the population. The average dose associated with each procedure used will be obtained from previous Australian surveys and estimation techniques using current software. Comparisons with other countries are demonstrated.

Results The number of CT procedures performed each year in Australia is increasing at an average rate of $8.6 \%$ per year where as the population is only increasing at an average rate of $1.3 \%$ per year. There are consistently more CT scans performed on women than there are on men in the proportions of $54-46 \%$. The rate of increase across individual scan categories varies with the greatest being Interventional CT scans which increased an average of $32 \%$ per year. The next categories with the highest rates of increase per year are Chest Angiography and Neck-Chest-Abdomen-Pelvis scans.

Conclusions The rate of increase in CT scans performed in Australia is increasing significantly faster than the population growth. While medical sources contribute $35 \%$ to the total radiation dose received by the population, the relatively high dose per CT procedure alone makes up $45 \%$ of these medical sources ${ }^{4}$. If the number of CT scans continues to increase at the current rate, the dose received by the public from medical sources will overtake the dose received from natural background radiation.

\section{References}

1. United Nations Scientific Committee on the Effects of Atomic Radiation, Medical Radiation Exposures 2000 Report Vol. 1, Annex D: Medical Radiation Exposures.

2. Department of Health and Aging.

3. EC RP No. 154, http://ec.europa.eu/energy/nuclear/radiation protection/publications_en.htm.

4. KN Wise JEM Thomson, (2004), 'Changes in CT radiation doses in Australia from 1994 to 2002.' The Radiographer, Vol. 51, No. 2 .

Effective atomic numbers of solid phantoms for low energy X-rays

Robin Hill ${ }^{1,2}$, Zdenka Kuncic ${ }^{1}$, Clive Baldock ${ }^{1}$

${ }^{1}$ Institute of Medical Physics, School of Physics, The University of Sydney, ${ }^{2}$ Department of Radiation Oncology, Royal Prince Alfred Hospital

Introduction Solid phantoms are routinely used for quality assurance testing and radiation dosimetry measurements in radiotherapy. In this work, we calculate the effective atomic number, $\mathrm{Z}_{\mathrm{eff}}$, for a number of solid phantoms using the X-ray spectra information and assess whether $Z_{\text {eff }}$ is an appropriate parameter for determining water equivalence.

Methods A total of ten solid phantoms were evaluated including solid water type phantoms, Perspex, polystyrene, PRESAGE and the PAGAT polymer dosimeter and additionally water. The fractional weights of the elements in the phantom were taken from the literature or the manufacturer's specifications. Calculations of $\mathrm{Z}_{\mathrm{eff}}$ were performed using the methodology of Manohara et al. for each of the phantoms [4]. This methodology was further developed by weighting the calculations using the primary photon spectrum of $\mathrm{X}$-ray beams with energies ranging from 50 to $280 \mathrm{kVp}$. The photon cross section data used was the XCOM database provided by the NIST.

Results and discussion $A$ graph of the values of $Z_{\text {eff }}$ is shown in the figure below. The Plastic Water had the largest $Z_{\text {eff }}$ values as compared to water while the values of $Z_{\text {eff }}$ for polystyrene were the smallest. Calculations of $Z_{\text {eff }}$ for several water equivalent solid phantoms including RMI-457 Solid Water, Virtual Water and Plastic Water DT were all consistently greater than those of water. The phantom materials with $Z_{\text {eff }}$ that were the closest to water were A150, PAGAT, PMMA and RW3. While the composition of PAGAT is very similar to water, both PMMA and RW3 have been shown to give significant differences in dose at low X-ray beam energies. 


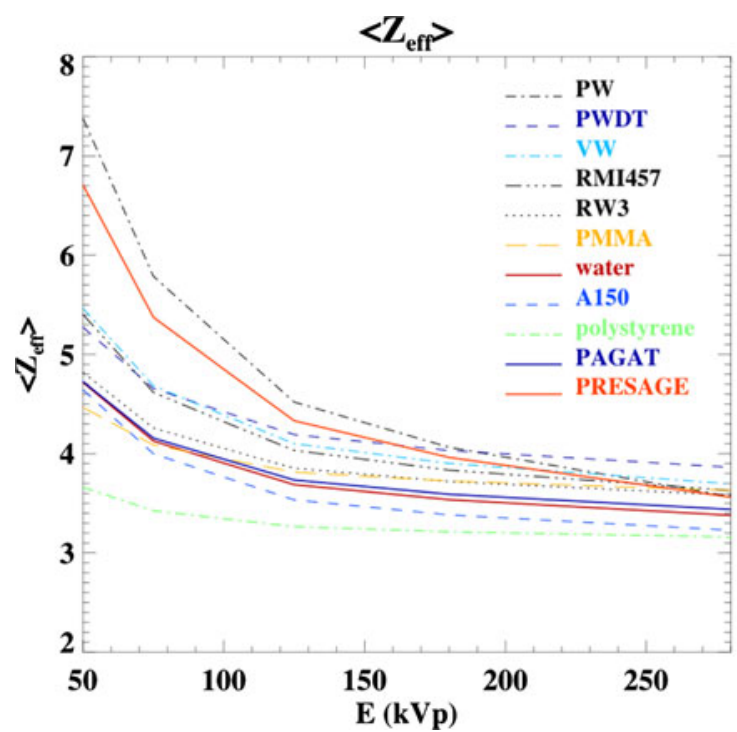

Conclusion Effective atomic number is not suitable parameter for determining water equivalence of a solid phantom.

\section{CTCombine: rotating and combining CT data with an accurate detector model to simulate radiotherapy portal imaging at non-zero beam angles}

Tanya Kairn ${ }^{1}$, Markus Dwyer ${ }^{2}$, David Warne ${ }^{2}$, Tim Markwell ${ }^{1}$, Jamie Trapp ${ }^{1}$, Andrew Fielding ${ }^{1}$

${ }^{1}$ School of Physical and Chemical Sciences, Queensland University of Technology, ${ }^{2}$ High Performance Computing, Queensland University of Technology

Established Monte Carlo user codes BEAMnrc and DOSXYZnrc permit the accurate and straightforward simulation of radiotherapy experiments and treatments delivered from multiple beam angles. However, when an electronic portal imaging detector (EPID) is included in these simulations, treatment delivery from non-zero beam angles becomes problematic. This study introduces CTCombine, a purpose-built code for rotating selected CT data volumes, converting CT numbers to mass densities, combining the results with model EPIDs and writing output in a form which can easily be read and used by the dose calculation code DOSXYZnrc.

The methodology behind CTCombine is based on Chin et al.'s TWIZ and GLU procedures [1] which simulate a $\theta^{\circ}$ gantry rotation by rotating the $\mathrm{CT}$ volume by $-\theta^{\circ}$, padding with it air and then running the NRC CT conversion code CTCREATE using the modified CT volume, before combining the resulting text file with a model of the EPID.

CTCombine simplifies and builds on these procedures by:

- combining all three steps (rotating the CT, converting CT numbers to density and adding the detector) within one simple program;

- introducing the ability to rotate around a user-defined isocentre (not necessarily at the centre of the CT-volume);
- introducing the ability to interpolate between CT slices when the desired Monte Carlo voxel size is smaller than the slice width;

- using affine transformations to minimise differences between the geometry of the patient before and after rotation;

- introducing the ability to select subsets of the CT data for inclusion in the final model (allowing couch removal);

- adapting CTCREATE to allow more complex CT-number-density relationships to be modelled.

The geometric and dosimetric accuracy of CTCombine's output is assessed by simulating simple and complex treatments applied to a rotated planar phantom and a rotated humanoid phantom and comparing the resulting virtual EPID images with the images acquired using experimental measurements and independent simulations of equivalent phantoms. It is expected that CTCombine will be useful for Monte Carlo studies of EPID dosimetry and will therefore become a valuable tool for radiotherapy treatment verification.

Acknowledgments This work is funded by the NHMRC, through a project grant. The authors wish to thank Darren Cassidy of the Royal Brisbane and Women's Hospital and Steve Sylvander and Emmanuel Baveas of Mater Radiation Oncology for assistance with CT scanning radiotherapy phantoms. The authors are also grateful to Elekta for the provision of manufacturing specifications which permitted the detailed simulation of their linear accelerators and amorphous-silicon electronic portal imaging devices. Computational resources and services used in this work were provided by the HPC and Research Support Group, Queensland University of Technology, Brisbane, Australia.

\section{Reference}

1. PW Chin et al, Phys. Med. Biol, 48: N231-N238 (2003).

Tissue equivalency of phantom materials for dosimetry of secondary neutrons in proton therapy

Stephen Dowdell ${ }^{1}$, Ben Clasie ${ }^{2}$, Andrew Wroe ${ }^{1,3}$, Susanna Guatelli ${ }^{1}$, Peter Metcalfe ${ }^{1}$, Reinhard Schulte ${ }^{3}$, Anatoly Rosenfeld ${ }^{1}$

${ }^{1}$ Centre for Medical Radiation Physics, University of Wollongong, ${ }^{2}$ Department of Radiation Oncology, Massachusetts General Hospital and Harvard Medical School, ${ }^{3}$ Department of Radiation Medicine, Loma Linda University Medical Center, USA

Introduction Previous studies involving secondary neutrons in proton therapy have employed a number of phantom materials which are designed to represent human tissue.

Methods Monte Carlo simulations were used to compare the absorbed dose and dose equivalent from secondary neutrons generated from $150 \mathrm{MeV}$ proton beams within common phantom materials and various ICRP tissues. Comparisons were performed both along the central axis and out of field. The phantom materials chosen for comparison were Lucite, liquid water, solid water and A150 tissue equivalent plastic. These phantom materials were compared to brain, muscle and adipose tissues.

Results The doses observed were smaller than those reported in previous studies which incorporated neutrons generated in the treatment head. For both neutron absorbed dose and dose equivalent, no phantom material gives agreement with each of the tissues within 5\% at all the points considered. 

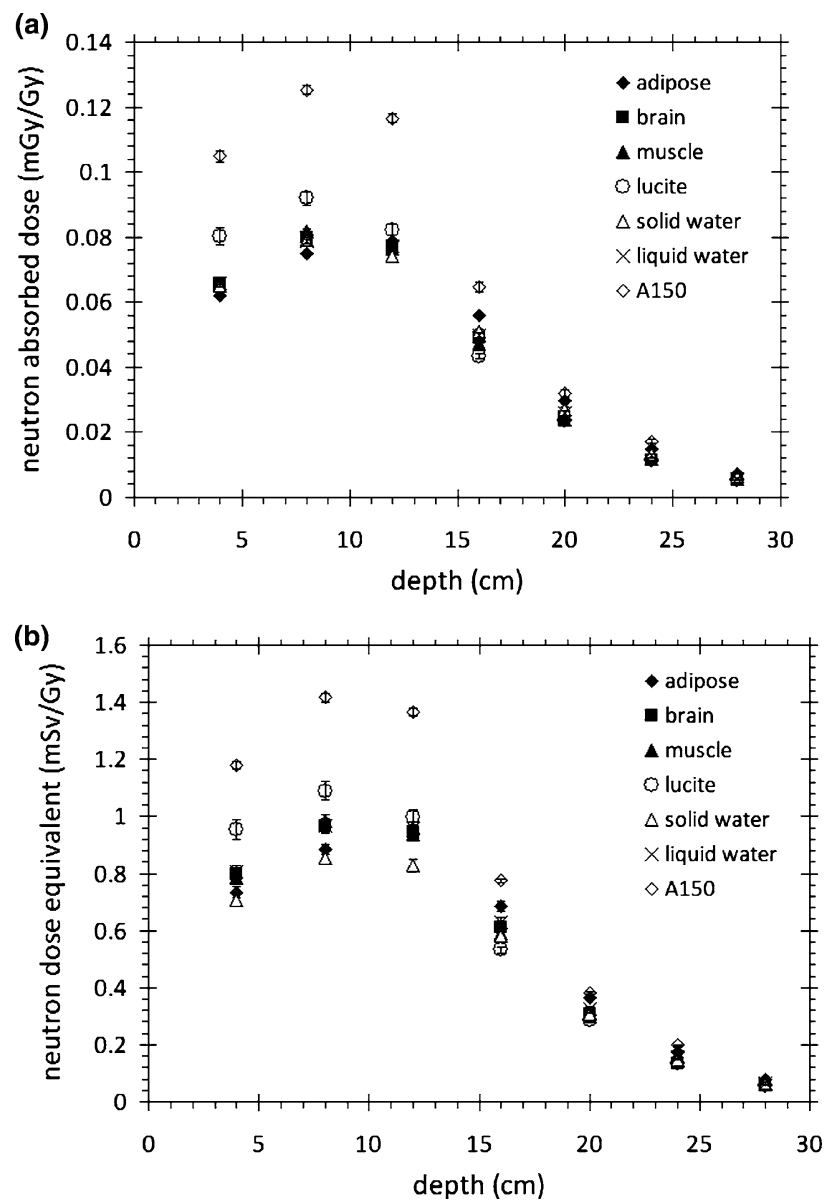

Fig 1 Neutron absorbed dose (a) and dose equivalent (b) along the central axis of the primary beam

Conclusion The most suitable phantom material for dosimetry of secondary neutrons in proton therapy may depend on the specific tissue type being investigated and the position of measurement.

\section{Commissioning electron MU using the Varian Eclipse electron Monte Carlo (eMC) algorithm}

Rebecca Murry ${ }^{1}$, Brendan Healy ${ }^{1}$

${ }^{1}$ Radiation Oncology Queensland

Monitor units (MU) for electron fields are currently determined in our department by applying an output factor that has been measured in a solid water phantom specifically for the treatment insert in question. The practicality of using Varian Eclipse-generated MU in a clinical setting was investigated.

MU for the electron fields were calculated by Eclipse (v8.6) using eMC (with settings: calculation grid $=0.2 \mathrm{~cm}$; particle histories $=100,000,000$; smoothing method $=$ no_smoothing). Four electron energies were considered $(6-16 \mathrm{MeV})$ for a range of insert sizes in each of five applicators using a cubic water phantom. Measured and eMC-predicted applicators factors were compared. A number of circular inserts was also chosen (across all energies and applicators) and the Eclipse MU was calculated.

Eclipse-calculated applicator factors agreed with measured values within $\pm 1 \%$. Eclipse MU for 6 and $9 \mathrm{MeV}$ inserts agreed within $\pm 1.5 \%$ while all but one insert size for 12 and $16 \mathrm{MeV}$ agreed within $\pm 3 \%$. Despite this good agreement we will continue to use our current method of calculating MU using an output factor measurement (or prediction of an output factor using a measurement library). Movement to the use of Eclipse MU would involve increased calculation time due to the need for having a 'smoothed' plan to visualise dosimetry and an 'unsmoothed' plan for determination of MU. Further work also needs to be done to validate the role of heterogeneity and obliquity in the eMC MU calculation.

Comparison of digitally reconstructed radiograph (DRR) generated from axial and helical scanning mode-a phantom study

Vinod Nelson ${ }^{1}$, Shrikant Deshpande ${ }^{1}$, Lois Holloway ${ }^{1}$, Phillip Vial ${ }^{1}$

${ }^{1}$ Macarthur Cancer Therapy Centre, Sydney South West Area Health Service

Purpose Digitally reconstructed radiographs (DRRs) play a vital role for verifying patient position for many radiotherapy treatments. As DRRs are generated from CT scans, image quality may be affected by scanning mode (axial or helical). This study aimed to investigate the significance of this variation, if any.

Methods and material A specifically designed CT simulation phantom (Nuclear Associates Model 76-417) was used to evaluate DRR characteristics for a Siemens Somatom Sensation4 CT scanner. This phantom contains test patterns to measure contrast, modulation transfer function (MTF), ray line divergence, and spatial distortion. The phantom was scanned as described in the manufacturer's manual, using the departmental protocol for both head and pelvis. For helical scanning four pitches (1-2) were used. Each of the test patterns was compared for the axial and helical scan.

Results The contrast values for DRRs ranged from 0.5337 to 4.9267 for axial scans and 0.4523 to 3.2768 for helical scans for hole diameters of $1 \mathrm{~mm}$ to $1 \mathrm{~cm}$, respectively. For hole depths from 0.5 to $2.5 \mathrm{~mm}$ contrast values ranged from 0.5202 to 5.5955 for axial scans and 0.4423 to 5.6325 for helical scans. Low contrast values obtained from different depth holes and diameter patterns do not show any trends. The calculated MTF showed higher spatial resolution for DRRs generated from axial scans compared with helical scans at pitches $>1.25(21.2 \%$ at $1 \mathrm{lp} / \mathrm{mm}$ for axial, $2.5 \mathrm{~mm}$ slice versus $14.5 \%$ for pitch 1.25), however no significant differences were seen compared with a helical scan with pitch of 1 .The ray line divergence and spatial linearity was comparable in DRRs from both scanning modes.

Conclusion The present study shows that DRRs obtained from helical scans with pitch 1 are comparable to DRRs obtained from axial scans. DRRs from helical scans with pitch $>1.25$, showed reduced spatial resolution compared with axial scans.

Skin dose increases in MRI guided radiotherapy: a Monte Carlo study

Bradley Oborn ${ }^{1}$, Peter Metcalfe ${ }^{1}$, Martin Butson ${ }^{1,2}$, Anatoly Rosenfeld ${ }^{1}$

${ }^{1}$ University of Wollongong, ${ }^{2}$ Illawarra Cancer Care Centre

Background MRI guided radiotherapy (MRIgRT) is a promising form of Image Guided Radiotherapy (IGRT). This modality is essentially the merging of a Medical Linac with an MRI scanner. Two groups have working prototypes and are pushing for clinical trials. The magnetic field of the MRI however causes skin dose changes that have not been previously studied at high resolution $(0.07-0.5 \mathrm{~mm}$ depth). Aims Determine accurate estimates of the skin dose $(0.07-0.5 \mathrm{~mm}$ depth) from MRIgRT. 
Methods GEANT4 Monte Carlo simulations of a Varian 2100c accelerator with transverse magnetic fields have been performed as described recently ${ }^{1}$. Voxels with 10 micron depth resolution have been employed to provide accurate estimates of the skin doses at the ICRU reference depth $(0.07 \mathrm{~mm})$ below the skin surface in virtual water phantoms.

Results Entry skin dose is decreased in MRIgRT for 0.4-2 Tesla systems due to the removal of lepton contamination. Exit skin dose is however always increased due to the Electron Return Effect (ERE); where electrons leaving the exit face are forced to return and deposit dose. Exit skin doses may increase by up to $106 \%$ depending on magnetic field strength and field size.

Conclusions The skin dose predicted by Monte Carlo simulations are of clinical concern in MRIgRT. This is primarily due to the ERE. Exit bolus will significantly offset the increases and so makes clinical MRIgRT more achievable.

\section{Reference}

1. Oborn et al, Med. Phys. 36(8), August 2009.

Use of an Epson scanner and an X-Rite densitometer for radiochromic film

\author{
Anna Ralston ${ }^{1}$, May Whitaker ${ }^{1}$, David Odgers ${ }^{1,2}$, Gwi Cho ${ }^{1}$ \\ ${ }^{1}$ Royal Prince Alfred Hospital, ${ }^{2}$ School of Physics, University of \\ Sydney
}

Introduction The optical density of radiochromic film can be measured either with a scanner, for 2-D dose mapping, or a point densitometer, which is fast and convenient for reading out small pieces of film. In this study an Epson 10000XL A3 scanner and an $\mathrm{X}$-Rite $361 \mathrm{~T}(\mathrm{X})$ point densitometer were commissioned for use with radiochromic film.

Methods EBT and EBT2 Gafchromic films were exposed to a $6 \mathrm{MV}$ photon beam with doses up to $3 \mathrm{~Gy}$. The Epson scanner was used in transmission mode, and the results were analysed with RIT software. The X-Rite densitometer was used with the $\times 10$ option for increased precision.

Results

\begin{tabular}{|c|c|c|}
\hline & EPSON 10000XL & X-RITE 361T(X) \\
\hline Spatial resolution & 72 dots per inch & $\begin{array}{l}\text { Depends on aperture } \\
\text { size selected } \\
(1,2 \text { or } 3 \mathrm{~mm} \\
\text { diameter })\end{array}$ \\
\hline $\begin{array}{l}\text { Optical density } \\
\text { resolution }\end{array}$ & $\begin{array}{l}48 \text { bits for full colour; } \\
16 \text { bits for red } \\
\text { channel alone }\end{array}$ & $\begin{array}{l} \pm 0.001 \text { O.D. when } \\
\text { used in } \times 10 \text { mode }\end{array}$ \\
\hline $\begin{array}{l}\text { Dependence on } \\
\text { film orientation }\end{array}$ & $\begin{array}{l}\text { Film must be oriented } \\
\text { the same way each } \\
\text { time. If not, error can } \\
\text { be } 5-10 \%\end{array}$ & No dependence \\
\hline $\begin{array}{l}\text { Placement of film } \\
\text { on scanner }\end{array}$ & $\begin{array}{l}\text { Significant variation in } \\
\text { scanner sensitivity } \\
\text { across scanner short } \\
\text { axis. Errors are up to } \\
2.6 \% \text { for a } 10 \mathrm{~cm} \\
\text { wide film, } 5 \% \text { for a } \\
15 \mathrm{~cm} \text { wide film, and } \\
7.2 \% \text { for a } 20 \mathrm{~cm} \\
\text { wide film. No } \\
\text { significant variation } \\
\text { for scanner long axis }\end{array}$ & N/A \\
\hline
\end{tabular}

\begin{tabular}{|c|c|c|}
\hline & EPSON 10000XL & X-RITE 361T(X) \\
\hline $\begin{array}{l}\text { Darkening of film by } \\
\text { light from device }\end{array}$ & $\begin{array}{l}\text { No darkening detected. } \\
\text { Films may be } \\
\text { scanned repeatedly } \\
\text { without being } \\
\text { affected }\end{array}$ & $\begin{array}{l}\text { There is darkening } \\
\text { of film by } \\
\text { densitometer light } \\
\text { source. The use of } \\
\text { a red filter reduces } \\
\text { this to } \\
\text { insignificant } \\
\text { levels }\end{array}$ \\
\hline
\end{tabular}

Discussion and conclusion The Epson scanner and the X-Rite point densitometer can provide precise and accurate measurements of radiochromic films, but they both have technical limitations which must be taken into account. In particular the positioning of the film on the scanner's glass plate is critical, and the point densitometer must be used with a red filter to avoid darkening of the film by the densitometer's light source.

\section{The effect of magnetron parameters on $\mathrm{X}$-ray beam properties}

Vince Reynolds ${ }^{1}$, Johny Morales ${ }^{2}$, Robin $\mathrm{Hill}^{2}$, Gwi-Ae Cho ${ }^{2}$

${ }^{1}$ Department of Biomedical Engineering, Royal Prince Alfred Hospital, ${ }^{2}$ Department of Radiation Oncology, Royal Prince Alfred Hospital

Introduction On a Varian 6EX (Varian Medical Systems, Palo Alto, USA) linear accelerator, changes in microwave power and the gun current have impact on the quality of X-ray beam. On this low energy unit, the microwave power is provided by the magnetron. In this work, we present the effect of magnetron input parameters on X-ray beam properties.

Methods During the service of the linear accelerator, the de-queuing reference voltage was adjusted and the yield servo balanced. These changes were performed in order to increase the RF power in order to increase the energy of the X-ray beam. Initially the de-queuing reference voltage was slowly increased and the beam profiles measured until they agreed to within $\pm 1 \%$ of the baseline scans taken during commissioning.

During the beam tuning, we found that the forward power from the waveguide was decreasing over time. This was attributed to magnetron filament voltage setting being on the unstable part of the magnetron power curve. Subsequently, the magnetron filament voltage was adjusted to manufacturer's recommendations. This was confirmed by performing beam profiles scans in a scanning water phantom.

Results The de-queuing reference voltage setting was adjusted from 3.455 to $3.66 \mathrm{~V}$ The final magnetron filament voltage was $13.34 \mathrm{~V}$ at which time the X-ray beam was stable. The final measurements of depth doses were better than $1 \%$ as compared commissioning data. We also achieved our goal of beam profiles being within $1 \%$ of commissioning data.

Conclusions The filament voltage has an impact on the stability of the $\mathrm{X}$-ray beam. The de-queuing reference voltage has a significant effect on the energy and the horns in the beam profiles.

Can we improve access to images for diagnosis and treatment and increase hospital efficiency by writing software to automate image handling? Challenges and consequences

Paul Roxby ${ }^{1}$, David Binns ${ }^{3}$, Emily Hong ${ }^{3}$, Cathie O'Shannessy ${ }^{2}$, Lal Peiris ${ }^{2}$, Trish Hubbard ${ }^{4}$, Colin Hornby ${ }^{4}$, Nilgun Touma ${ }^{5}$, $\mathrm{Li} \mathrm{Zhu}^{5}$, Jim Cramb ${ }^{1}$ 
${ }^{1}$ Physical Sciences, Peter MacCallum Cancer Centre, ${ }^{2}$ Diagnostic Imaging, Peter MacCallum Cancer Centre, ${ }^{3}$ Molecular Imaging, Peter MacCallum Cancer Centre, ${ }^{4}$ Radiation Therapy Services, Peter MacCallum Cancer Centre, ${ }^{5}$ Tattersalls Cancer Centre, Epworth Hospital

Purpose Having access to prior images and a range of imaging modalities is important in cancer diagnosis and staging and in radiotherapy treatment planning. Considerable time was being spent importing images from $\mathrm{CD}$ and changing patient details to those of the receiving hospital. Typing new details could introduce errors.

Sending PET images to other hospitals over network links required manual steps and staff time.

In radiotherapy treatment a digital graticule was provided on an electronic portal imaging system but not on the record and verify system which stores the images for later review. This required entering the room to add a manual graticule during treatments.

Methods For image export from a PET imaging centre, single click export of images to other hospitals was provided by aranging for a DICOM destination to create zip files, upload these to a secure web site and send an email.

For image import, software was written to find DICOM files from a $\mathrm{CD}$, correct problems where possible, offer a list of matching patient details and DICOM worklist entries, change patient details and send to a PACS.

For radiotherapy imaging, changing the manufacturer and model tags in the DICOM image activated additional functionality in the record and verify system to display the image centre, and enabled an overlay graticule to be displayed.

Results These successful projects encountered technical and political challenges and entailed a commitment to provide an ongoing, reliable service. They have improved relations with other departments through saving staff time.

\section{Spinal canal segmentation with well-balanced speed and accuracy combination for radiation treatment planning}

Akira Sawada $^{1}$, Yukinori Matsuo ${ }^{1}$, Masaru Narabayashi ${ }^{1}$, Masahiro Hiraoka ${ }^{1}$

${ }^{1}$ Department of Radiation Oncology and Image-Applied Therapy, Kyoto University Graduate School of Medicine

Purpose The purpose of this study is to propose a fast automatic spinal canal segmentation algorithm which is clinically acceptable in terms of speed and accuracy.

Method and materials The method consists of $2 \mathrm{D}$ and 3D extraction phases. In the $2 \mathrm{D}$ extraction phase, smoothing and multilevel thresholding are performed for each ROI (Region Of Interest) in CT images. Next, erosion followed by dilation of the extracted blobs is applied to eliminate speckle-shaped and thin line-shaped blobs. Subsequently, island blobs having areas less than a predetermined threshold level are removed. Then, the compactness indicating a disk-shape is calculated for each blob and the blob with the maximum compactness is extracted. In the 3D extraction phase, false regions are removed by evaluating variations of the centroid positions of the identified blobs in the slice direction. For improperly extracted ROI images, interpolation in the slice direction is performed by referring to the properly extracted blobs in the adjacent images.

Results Thirty-two patients with lung cancer were enrolled in this study. The false blobs in 2D extraction phase are replaced to reasonable ones in 3D extraction phase and smoother shape was clearly observed in 3D views. The median accuracy was $90 \%$ within the range of $77-100 \%$ on clinical acceptance by two radiation oncologists. The computation time was approximately $6 \mathrm{~s}$ for 126 slices.
Conclusion Verification results have showed that the proposed method is well-balanced speed and accuracy combination, which expectedly leads to reduction of oncologist's workload for radiation treatment planning.

\section{Radiation dose in the 3D spectroscopic MARS scanner}

Stefanie Girst ${ }^{1,5}$, Nanette Schleich ${ }^{1,2}$, Nick Cook $^{2}$, Hansjörg Zeller ${ }^{1}$, Anthony Butler ${ }^{1,3,4}$, Phil Butler ${ }^{1,4}$

${ }^{1}$ Department of Physics and Astronomy, University of Canterbury, New Zealand, ${ }^{2}$ Medical Physics and Bioengineering, Canterbury District Health Board, New Zealand, ${ }^{3}$ Christchurch School of Medicine, University of Otago, New Zealand, ${ }^{4}$ European Organisation for Nuclear Research, Geneva, Switzerland, ${ }^{5}$ Technical University of Munich, Germany

Introduction The Medipix All Resolution System (MARS) is a novel X-ray imaging CT scanner based on the Medipix photon processing detector. Relative to conventional CT scanners, the characteristics of the 3D spectroscopic imaging detector offer improved sensitivity and resolution as well as reduced patient dose, e.g. through reducing the need of repeat scans with contrast media. In this paper, the results of a study to identify practical radiation dose indicators for MARS, including CTDI $_{\text {air }}$ and DLP, are discussed.

Methods TLD, solid state detector, ionisation chamber and MonteCarlo simulations are possible methods for dose characterisation. This study focused on TLDs and a solid state detector, as these were the most practicable and convenient means of dose measurement. Radiation output and doses beneath varying thicknesses of PMMA were measured with an Unfors Xi R/F and MAM Detector. Calculations of surfaces doses to the phantom were performed. LiF TLDs were exposed at the centre of a PMMA phantom of an identical design to the cylindrical imaging geometry being used. The TLDs were calibrated utilising a linear accelerator. The results of both methods were compared with the results of Monte Carlo simulations of the complete scanner system.

Conclusions TLDs are well suited for relative dose measurements. However, the calculation of radiation dose to water is complicated by the low effective energy of the MARS X-ray beam and the consequent departure of the TLDs from behaving as a large cavity detector. The Unfors $\mathrm{Xi}$ R/F and MAM Detector offers convenience, but cannot easily be used for a phantom measurement due to its size and the short focal spot to detector distance.

\section{Dosimetric verification of a treatment chain using TRS 398 protocol}

Hyong Seo ${ }^{1}$, Philip Satory ${ }^{1}$, Jonathan Downs ${ }^{1}$, Christine Thompson ${ }^{1}$, Allan Stewart ${ }^{1}$

\section{${ }^{1}$ Cancer and Blood Services, Auckland City Hospital, New Zealand}

The purpose of this work was to evaluate the accuracy of the whole treatment system including the CT, treatment planning system (Pinnacle, Version 8.0), treatment setup and delivery method currently implemented in Auckland City Hospital. Three treatment plans were generated to emulate an off axis chest, breast and oesophagus treatments using $6 \mathrm{MV}$ beams. 2 Gy per fraction was prescribed to the centre of the chamber within the water phantom. The dose was measured using the NRL (National Radiation Laboratory, Christchurch) certified graphite chamber and were converted to dose in Gy using the TRS 398 protocol. Measured doses were corrected for the daily machine output for comparison with the Pinnacle calculated dose. Measured doses were 1.1, 0 and $1.5 \%$ higher than the Pinnacle calculated dose upon applying daily machine output corrections. The maximum dose to the Planning Target Volume (PTV) should be 
delivered within $\pm 3 \%$ according to published data. Our results from three plans fell within this tolerance which leaves maximum of $1.5 \%$ error for the inter-fractional setup movements and inter-fractional changes in daily machine outputs that were not accounted for in this experiment.

The role of audit and feedback in achieving sustained change in clinician behaviour: application to radiation use reduction in coronary angiography

Ian Smith $^{1}$, John Rivers ${ }^{1}$, James Cameron ${ }^{1}$, Russell Brighouse ${ }^{1}$, Kelley Foster ${ }^{1}$

${ }^{1}$ St Andrew's War Memorial Hospital

Introduction Education is often a dominant requirement of most programs aimed at modifying clinician performance. Behaviour modification theories (BMT) suggest that reliance on a single intervention strategy is flawed. This study uses BMT to explain the need for education to be supported by detailed audit + feedback to optimally modify clinician radiation use in coronary angiography. Although a theoretical understanding of effective radiation hygiene principles had some impact on radiation use, our experience suggests that an appropriately managed audit + feedback program was essential in achieving long term sustained reductions.

Methods Radiation use records (procedure fluoroscopy time) for ten cardiologists using the SAWMH CCT from July 1996 to December 2005 were analysed to assess the impact of various interventions aimed at minimising radiation risk. To reduce the impact of case complexity variations, only data for diagnostic coronary angiograms having a minimal disease burden were analysed. The impact of interventions such as education and audit + feedback were correlated against Cumulative Sum and Cumulative Expected minus Observed charts.

Results Group performance charts did not show any marked improvement in radiation use associated with education alone. However, performance improved significantly once regular detailed feedback providing each operator with their comparative radiation use was provided. Charts for individual users showed the time frame for response differed from person to person.

Conclusion Understanding the psychology around confidential performance reporting is critical to implementing programs aimed at improving clinical practice. Regular detailed quantitative performance auditing and feedback to support education was effective in reducing radiation use in coronary angiography.

Peripheral photon and neutron doses from prostate cancer external beam irradiation and the risk of second primary cancers

R Takam ${ }^{1,2}$, E Bezak $^{1,2}$, EE Yeoh ${ }^{3}$, G Liu $^{2}$

${ }^{1}$ School of Chemistry and Physics, The University of Adelaide, ${ }^{2}$ Medical Physics Department, Royal Adelaide Hospital, ${ }^{3}$ Radiation Oncology Department, Royal Adelaide Hospital

Purpose To measure peripheral photon and neutron doses in Rando phantom and estimate the associated risks of second primary cancer in various organs distal to the target volume as a result of prostate carcinoma external beam radiotherapy using $18 \mathrm{MV}$ X-ray beam from Varian iX medical linear accelerator.

Method ${ }^{6} \mathrm{LiF}: \mathrm{Mg}, \mathrm{Cu}, \mathrm{P}$ and ${ }^{7} \mathrm{LiF}: \mathrm{Mg}, \mathrm{Cu}, \mathrm{P}$ glass-rod thermoluminescent dosimeters (TLDs) were inserted in slices of Rando phantom and followed by irradiation of the phantom to $80 \mathrm{~Gy}$ radiation dose using 4-field 3D-CRT technique and $18 \mathrm{MV} \mathrm{X}$-ray beam. ${ }^{6} \mathrm{LiF}: \mathrm{Mg}, \mathrm{Cu}, \mathrm{P}$ and ${ }^{7} \mathrm{LiF}: \mathrm{Mg}, \mathrm{Cu}, \mathrm{P}$ TLDs were calibrated using 6 and $18 \mathrm{MV}$ X-ray beams. Neutron dose equivalents measured with CR-39 etch-track detectors were used to derive readout-to-neutron dose conversion factor for ${ }^{6} \mathrm{LiF}: \mathrm{Mg}, \mathrm{Cu}, \mathrm{P}$ TLDs.

Results The average total (photon + neutron) dose equivalents per $1 \mathrm{~Gy}$ isocentre dose range from $4.0 \pm 0.9 \mathrm{mSv} / \mathrm{Gy}$ (thyroid) to $15.0 \pm 13.9 \mathrm{mSv} / \mathrm{Gy}$ (colon). The associated risks of second primary cancer estimated using the competitive risk model were found to range from $1.5 \pm 0.3 \%$ (thyroid) to $4.5 \pm 4.2 \%$ (colon).

Conclusion Radiotherapy of prostate carcinoma using EBRT technique and high-energy linear accelerator is associated with elevated risk of second primary cancer in distal organs.

Dosimetric characteristics and potential applications of the ImRT MatriXX operating in movie mode

Philip Vial $^{1,2}$, Shrikant Deshpande ${ }^{1}$, Gary Goozee ${ }^{1}$, Lois Holloway ${ }^{1,2,3}$

${ }^{1}$ Department of Medical Physics, Liverpool and Macarthur Cancer Therapy Centres, ${ }^{2}$ Institute of Medical Physics, School of Physics, University of Sydney, ${ }^{3}$ Centre for Medical Radiation Physics, University of Wollongong

Purpose Standard detectors are not suited for time resolved measurements of linear accelerator (linac) beams. The purpose of this study was to benchmark the dose response of a new detector operating in movie mode with fast frame rates for time resolved measurements of linac beams.

Methods and materials The ImRT MatriXX (IBA Dosimetry GmbH, Schwarzenbruck) ionisation chamber array was operated in 'movie' mode with frame rates up to $50 \mathrm{~Hz}$. The signal dead time, precision, and signal to noise ratio (SNR) was investigated. Several different radiation sources were measured. The possibility of measuring dose from individual linac pulses was investigated, as was the ability to make time resolved measurements of beams with frequent beam gating.

Results No dead time was observed. The small signal measured during short frame periods have relatively poor precision and SNR. For linac measurements, to maintain noise levels at less than $1 \%$ using frame rates of $50 \mathrm{~Hz}$ it was necessary to position the detector at $65 \mathrm{~cm}$ from the source and use dose rates of $300 \mathrm{MU}$ per minute or greater. Aliasing between linac beam pulses and frames was observed. By reducing the linac's pulse repetition frequency it was possible to isolate individual beam pulses but with poor SNR. Dose rate fluctuations of gated beams were observed.

Conclusion The dose response of the MatriXX in movie mode at $50 \mathrm{~Hz}$ was accurate and reliable within the constraints of SNR. This detector has the potential to provide new insights into linac beam characteristics.

A software program to determine possible fractionation schedules tailored for individual patients based on normal tissue tolerances Lois Holloway $^{1,2,3}$, Philip Vial ${ }^{1,2}$

${ }^{1}$ Department of Medical Physics, Liverpool and Macarthur Cancer Therapy Centres, ${ }^{2}$ Institute of Medical Physics, School of Physics, University of Sydney, ${ }^{3}$ Centre for Medical Radiation Physics, University of Wollongong

Purpose Radiotherapy dose escalation has been shown to improve overall survival in some malignancies. Dose escalation is limited by the adverse effect to normal tissues. Effect to normal tissues varies with individual patient dose distributions. The goal of this study was to develop a software program to determine potential increase in fraction number or size based on individual patient plans. 
Materials and methods The software was developed using MatLab (Mathworks inc.). The inputs are dose volume histograms (DVHs) for the target and normal tissues for the given plan, acceptable normal tissue model values or DVHs and intended target dose prescription. Possible increases in fraction size or number are calculated according to the linear quadratic model. Normal tissue tolerance is assessed with EUD (Niemierko), Lyman Kutcher Burman NTCP and/or the relative seriality NTCP. The program was tested using a series of Head and neck and a series of prostate patient data sets. The results were verified by independent calculations.

Results and discussion Possible dose escalation schedules varied significantly for individual patients using different normal tissue tolerance models. The EUD model generally results in less dose escalation while the NTCP models resulted in greater escalation. Results also varied significantly between different patients for a given normal tissue tolerance model

Conclusions A program has been written and verified to determine possible increase in fraction number or size based on normal tissue tolerance. This program may be utilised to show possible variations in fractionation schedules for individual patient plans.

\section{Prostate hypofractionation: a modelling study considering IMRT treatment plans}

Tai Tran ${ }^{1,2}$, Lois Holloway ${ }^{1,2,3}$, Shivani Kumar ${ }^{2}$, Kirrily Cloak ${ }^{2}$, Mark Sidholm ${ }^{2}$, Karen Wong ${ }^{2}$, Philip Vial ${ }^{1,2}$

${ }^{1}$ Institute of Medical Physics, School of Physics, University of Sydney, ${ }^{2}$ Department of Radiation Oncology, Liverpool and Macarthur Cancer Therapy Centres, ${ }^{3}$ Centre for Medical Radiation Physics, University of Wollongong

Purpose The effects of fractionation are influenced by the tissue type $(\alpha / \beta$ values $)$ and dose distributions. This study has been undertaken to determine the possible hypofractionation for prostate cancer treatments for IMRT plans based on effects to normal tissues considering two $\alpha / \beta$ values.

Material and methods The Lyman, Kutcher Burman NTCP model, the relative seriality model and the EUD model were used to determine a single dose value representing the dose to bladder and rectum for a conventional treatment plan. The Linear Quadratic model, utilising Biological Effective Dose $(B E D=D \times(1+d /(\alpha / \beta))$ was then used to determine potential hypofractionation from an original 35 , 2 Gy fractions to the target, for the IMRT plan without exceeding these values. This was considered for both $\alpha / \beta=1.5 \mathrm{~Gy}$ and $\alpha /$ $\beta=10$ Gy for the prostate, values of 3.0 and 3.9 were used for the bladder and rectum respectively.

Results and discussion Modelling showed that the number of fractions could be reduced to 11-25 fractions. The EUD model showed the greatest change in fraction number and the LKB NTCP model the least. The biological effect to the rectum was more limiting than that to the bladder, however the difference was small. The change in fraction number was greatest for $\alpha / \beta=1.5 \mathrm{~Gy}$, although the difference was small.

Conclusions This modelling shows that with the introduction of IMRT, hypofractionation could be achieved without exceeding normal tissue reactions. The potential hypofractionation is greater if the $\alpha / \beta$ value for prostate is low, however there are also gains if the value is high.

\section{Comparison of dose from kilovoltage and megavoltage cone beam computed tomography}

Sankar Arumugam ${ }^{1,3}$, Lois Holloway ${ }^{1,2}$, Gary Goozee ${ }^{1}$,

J Velmurugan $^{3}$, Philip Vial ${ }^{1}$

${ }^{1}$ Department of Medical Physics, Liverpool and Macarthur Cancer Therapy Centres, ${ }^{2}$ Institute of Medical Physics, University of Sydney, ${ }^{3}$ Anna University, India
Purpose Highly conformal radiotherapy techniques require accurate patient positioning. Cone beam computed tomography (CBCT) can improve this at the cost of increased dose to the patient. The purpose of this work was to compare the dose delivered from $\mathrm{kV}$ and $\mathrm{MV}$ CBCT.

Methods and materials TLDs were used to determine the dose to the chest, pelvis and head and neck region of an anthropomorphic phantom for both $\mathrm{kV}$ and MV CBCT scans. An Elekta-Synergy Linear Accelerator (LA) with kV XVI imaging system and a Siemens-Oncor LA with MV-CBCT facility were used for the CBCT scans. Factory pre-sets were used for the $\mathrm{kV}$ images while a $6 \mathrm{MU}$ protocol was used for the head and neck region and an $8 \mathrm{MU}$ protocol for the pelvis region for the MV images.

Results The skin dose that resulted from $\mathrm{kV}$-CBCT imaging of thorax and pelvis regions ranged from 1.8 to $2.3 \mathrm{cGy}$.The dose inside the phantom varied between 2.2 and $3.3 \mathrm{cGy}$. In head and neck scanning the skin dose varied between 0.4 and $1.7 \mathrm{mGy}$. The dose measured at different anatomical sites was in the range from 0.4 to $1.3 \mathrm{mGy}$.

The skin dose that resulted from MV-CBCT imaging of thorax and pelvis regions ranged from 2.2 to $5.4 \mathrm{cGy}$. The dose at different points of the anatomical sites varied between 2.2 and $8.3 \mathrm{cGy}$. In Head and Neck scanning the measured skin dose ranged from 3.6 to $4.9 \mathrm{cGy}$ and the dose at different points inside the phantom was in the range form 4.2 to $6.4 \mathrm{cGy}$.

Conclusion A comprehensive set of dose measurements that resulted from $\mathrm{MV}$ and $\mathrm{kV}$ CBCT verification procedures was performed using anthropomorphic phantom.

\section{Scattering effect on CT numbers of CBCT images}

\section{Chuan-Dong Wen ${ }^{1}$}

${ }^{1}$ WP Holman Clinic, Royal Hobart Hospital

Introduction Cone beam CT (CBCT) has been clinically implemented in our department for $3 \mathrm{D}$ position verification when patient undergoes IMRT treatment. As CBCT images were generated differently from those of conventional fan beam CT, its CT number (CT\#) dependency on internal scattering within the cone need be well understood before CBCT data can be used for dosimetric purpose. This study presents our preliminary investigation on scattering effect of CT\# variation of Varian CBCT system at Royal Hobart Hospital. Methods A disk-type phantom ${ }^{1}$ (ScanPlas ${ }^{\circledR}$, Greenlands UK) of $33 \mathrm{~cm}$ diameter and $6 \mathrm{~cm}$ thickness of solid water with inserts of several different materials of human tissue equivalent at know electron densities ${ }^{2}$ was used to determine the corresponding CT\# for each material in this study. This phantom was scanned with CBCT system ${ }^{3}$ version 2.1.5.2Varian Medical Systems at $125 \mathrm{kV} 80 \mathrm{~mA}$ fitted with a half-fan filter for enhancing the beam quality and the choice of scan mode was a single cone of $45 \mathrm{~cm}$ wide and variable length capable of human pelvic site. The images were reconstructed using $512 \times 512$ pixel resolution and $1 \mathrm{~mm}$ slice thickness. By changing the length of the cone shape X-ray beam from 1 to $16 \mathrm{~cm}$, a series image set of the same phantom at identical set-up were produced. CT\# of each insert representing different densities was obtained by sampling over a circle of $2 \mathrm{~cm}$ in diameter at the centre of insert on the central slice of each image set. The insert's CT\# dependency upon the scatter variation caused by different scan volume can be revealed through plotting CT\# against scan length in centimetre. Pinnacle ${ }^{3}$ version $8.0 \mathrm{~m}$-Philips Medical Systems was used for CT\# analysis in this study.

Results The preliminary result indicated that CT\# of low density material (Lung-LN10/75) increased linearly with the length of cone beam up to the phantom thickness, in contrast those of higher density materials decreased with the length of scan cone. In particular, the $\mathrm{CT} \#$ for cortical bone (SB5) decreased at higher rate as the cone 
length increased. The extrapolated values for each material toward zero lengths agreed well with those produced by conventional fan beam CT.

Discussion and conclusions Due to complex scattering within the cone beam, CBCT CT\# depends on scan volume of the cone. CT\# of different material densities varies differently. CT\# to electron density correlation need be established at an appropriate clinical CBCT scan length and considered the scatter effect. Artefacts will be exhibited at a scan length threshold. It is noticed that short cone beam produced better CT\# correlation with those of fan beam CT and such image of short cone length could be used for clinical dosimetry application.

\section{References}

1. RA Chappell, Establishment of Relationship between CT Number and relative electron density, internal report to WP Holman Clinic, Royal Hobart Hospital, March 31, 1995.

2. ICRU Report 44 Tissue Substitutes in Radiation Dosimetry Measurement Appendix A 1989.

3. On-Board Imager (OBI) Reference Guide-Varian Medical Systems Ver 1.3 March 2007.

\section{Dosimetric evaluation of CBCT images for adaptive IMRT/IGRT treatment planning}

\section{Chuan-Dong Wen}

${ }^{1}$ WP Holman Clinic, Royal Hobart Hospital

Introduction Since the implementation of cone beam CT (CBCT) to verify treatment position for cancer patients undergoing IGRT/IMRT in our centre, the 3D CBCT data become readily available for an adaptive planning and treatment process. CBCT have advantages over conventional $\mathrm{CT}$ on reflecting patient internal organ temporal movement and inter-fractional treatment volume changes during tight-margined IMRT treatment course. This study presents our preliminary dosimetric evaluation of clinical CBCT images for adaptive planning with Pinnacle ${ }^{3}$ - Philips Medical Systems treatment planning system (TPS) at Royal Hobart Hospital.

Methods A series phantoms studies have been carried out using similar setup parameters on both CBCT and conventional CT systems to verify the characteristics of CT numbers of different materials and different scan modes, to examine the uniformity in axial plane and linearity along scan axis, and to establish the correlation of CT\# to density in the CBCT image dataset. Patient-specific CBCT data was imported into Pinnacle ${ }^{3}$ TPS and co-registered with the original planning CT data, before dose computation was carried out using CBCT data. Dose distributions in both phantom and patient were analysed and compared on both $\mathrm{CBCT}$ and $\mathrm{CT}$ datasets using Pinnacle $^{3}$ version $8.0 \mathrm{~m}$. CBCT data used in this study was acquired by Varian Medical Systems CBCT version 2.1.5.2.

Results Scans on uniformity phantoms indicated that CT\# uniformity and linearity on axial slices were acceptable in two scan modes capable of head and neck, and pelvis, fitted with full fan (FF) and half fan (HF) filters, except a crescent-shaped artefact with CT\# variation of more than $40 \mathrm{HU}$ emerged when scan length was beyond a threshold value. Artefacts displayed on both phantom and clinical images due to complex internal scatter within the cane beam and were depended on scan volume. Dose distributions in homogenous phantom suggested CBCT data can be used for dose calculation when scan length was relatively short and scatter from medium outside cone were adequate. CT\# to tissue density correlation was established through inter-comparison of CT/CBCT scans of ScanPlas ${ }^{\circledR}$ phantom and this relationship was scan volume dependent.

Discussion and conclusions CBCT dosimetric characteristics are more complex in comparing with of those of CT due to scattering effect within its scan volume. Various artefacts exist on some scan modes but can be minimised by optimising scanning parameters. CBCT data can be used with caution for clinical plan verification and validation in an adaptive IMRT process provided proper CT\# to density table has been established and the scan parameters have been correctly applied.

\section{References}

1. A quality assurance program for the on-board imager ${ }^{\circledR}$ Sua Yoo et al Med. Phys 33 (11), Nov 2006.

2. On-Board Imager (OBI) Reference Guide-Varian Medical Systems Ver 1.3 March 2007.

3. Catphan ${ }^{\circledR} 500$ and 600 Manual Copyright ${ }^{\odot} 2006$ the Phantom Laboratory.

4. Establishment of Relationship between CT Number and relative electron density, R A Chappell, internal report to W P Holman Clinic, Royal Hobart Hospital, March 31, 1995.

\section{Implementation of frame-based stereotactic radiosurgery system}

Timothy Williams ${ }^{1}$, Daniel Ramm ${ }^{1}$, John Lawson ${ }^{1}$, Raelene Nelligan ${ }^{1}$

${ }^{1}$ Royal Adelaide Hospital

Recently the existing Fischer-Liebinger frame based Stereotactic Radiosurgery (SRS) system at the Royal Adelaide Hospital was decommissioned and replaced with the BrainLAB SRS frame system. As SRS cone applicators were being used small field measurements were necessary. Output factor measurements were made with both PTW pin point chamber and small chip TLDs, the output factors were then compared and a selective combination of both measurements entered into the planning system. Once the beam data was measured and entered various tests were performed against the old FischerLiebinger system. Comparing simple beam arrangements found dose agreement at isocentre between the two systems to be within $2 \%$. Various verification measurements were made with differing complexity, ranging from simple point dose measurements in phantom through to the use of a SRS head phantom. The SRS head phantom was fixed in the headring and progressed from CT to treatment, testing not only the dosimetric accuracy of the system but also positional accuracy and transfer of the treatment parameters from the planning system to the treatment machine. RadCalc was successfully configured so that it can be utilised as a secondary MU checker for the BrainLAB arc treatments. The BrainLAB system is now in clinical use, and although still being used with SRS cones and patient headring, the new planning software has greatly enhanced the quality of service offered to patients. It is envisaged in the near future that the cones will be replaced with dynamic MLC treatments. 